\title{
Conformal metrics with constant $Q$-curvature for manifolds with boundary
}

\author{
Cheikh Birahim Ndiaye
}

\begin{abstract}
In this paper we prove that, given a compact four-dimensional smooth Riemannian manifold $(M, g)$ with smooth boundary, there exists a metric in the conformal class $[g]$ of the background metric $g$ with constant $Q$-curvature, zero $T$-curvature and zero mean curvature under generic conformally invariant assumptions. The problem is equivalent to solving a fourth-order non-linear elliptic boundary value problem (BVP) with boundary condition given by a third-order pseudodifferential operator and homogeneous Neumann condition. It has a variational structure, but since the corresponding Euler-Lagrange functional is in general unbounded from above and below, we need to use min-max methods combined with a new topological argument and a compactness result for the above BVP.
\end{abstract}

\section{Introduction}

In the last decades, much work has been done in the study of conformally covariant differential (pseudodifferential) operators on compact smooth Riemannian manifolds with smooth boundary, their associated curvature invariants, the corresponding boundary operators and curvatures in order to understand the relationships between analytic and geometric properties of such objects.

A model example is the Laplace-Beltrami operator on compact surfaces with boundary $(\Sigma, g)$ and the Neumann operator on the boundary. Under a conformal change of metric, the couple constitued by the Laplace-Beltrami operator and the Neumann operator governs the transformation laws of the Gauss curvature and the geodesic curvature. In fact, under the conformal 
change of metric $g_{u}=e^{2 u} g$, we have

$$
\left\{\begin{array} { l } 
{ \Delta _ { g _ { u } } = e ^ { - 2 u } \Delta _ { g } , } \\
{ \frac { \partial } { \partial n _ { g _ { u } } } = e ^ { - u } \frac { \partial } { \partial n _ { g } } , }
\end{array} \text { and } \quad \left\{\begin{array}{l}
-\Delta_{g} u+K_{g}=K_{g_{u}} e^{2 u} \text { in } \Sigma, \\
\frac{\partial u}{\partial n_{g}}+k_{g}=k_{g_{u}} e^{u} \text { on } \partial \Sigma
\end{array}\right.\right.
$$

where $\Delta_{g}$ (resp. $\Delta_{g_{u}}$ ) is the Laplace-Beltrami operator of $(\Sigma, g)$ (resp. $\left.\left(\Sigma, g_{u}\right)\right)$ and $K_{g}$ (resp. $\left.K_{g_{u}}\right)$ is the Gauss curvature of $(\Sigma, g)$ (resp. of $\left(\Sigma, g_{u}\right)$ ), $\frac{\partial}{\partial n_{g}}\left(\right.$ resp. $\left.\frac{\partial}{\partial n_{g_{u}}}\right)$ is the Neumann operator of $(\Sigma, g)$ (resp. of $\left.\left(\Sigma, g_{u}\right)\right)$ and $k_{g}$ (resp. $\left.k_{g_{u}}\right)$ is the geodesic curvature of $(\partial \Sigma, g)$ (resp. of $\left.\left(\partial \Sigma, g_{u}\right)\right)$. Moreover, we have the Gauss-Bonnet formula which relates $\int_{\Sigma} K_{g} d V_{g}+\int_{\partial \Sigma} k_{g} d S_{g}$ and the topology of $\Sigma$

$$
\int_{\Sigma} K_{g} d V_{g}+\int_{\partial \Sigma} k_{g} d S_{g}=2 \pi \chi(\Sigma)
$$

where $\chi(\Sigma)$ is the Euler-Poincaré characteristic of $\Sigma, d V_{g}$ is the element area of $\Sigma$ and $d S_{g}$ is the line element of $\partial \Sigma$. Thus, $\int_{\Sigma} K_{g} d V_{g}+\int_{\partial \Sigma} k_{g} d S_{g}$ is a topological invariant, hence a conformal one.

There exists also an other example of conformally covariant differential operator on four-dimensional compact smooth Riemannian manifolds with smooth boundary called the Paneitz operator and to which is associated a natural concept of curvature. This operator discovered by Paneitz in 1983 (see [19]) and the corresponding $Q$-curvature introduced by Branson [3] are defined in terms of Ricci tensor $\mathrm{Ric}_{g}$ and scalar curvature $R_{g}$ of the manifold $(M, g)$ as follows:

$$
\begin{aligned}
P_{g}^{4} \varphi & =\Delta_{g}^{2} \varphi+\operatorname{div}_{g}\left(\left(\frac{2}{3} R_{g} g-2 R i c_{g}\right) d \varphi\right) \\
Q_{g} & =-\frac{1}{12}\left(\Delta_{g} R_{g}-R_{g}^{2}+3\left|\operatorname{Ric}_{g}\right|^{2}\right)
\end{aligned}
$$

where $\varphi$ is any smooth function on $M, \operatorname{div}_{g}$ is the divergence and $d$ is the De Rham differential.

Likewise Chang and Qing, see [6], have discovered a boundary operator $P_{g}^{3}$ defined on the boundary of compact four-dimensional smooth Riemannian manifolds and a natural third-order curvature $T_{g}$ associated to $P_{g}^{3}$ as 
follows:

$$
\begin{aligned}
P_{g}^{3} \varphi= & \frac{1}{2} \frac{\partial \Delta_{g} \varphi}{\partial n_{g}}+\Delta_{\hat{g}} \frac{\partial \varphi}{\partial n_{g}}-2 H_{g} \Delta_{\hat{g}} \varphi+\left(L_{g}\right)_{a b}\left(\nabla_{\hat{g}}\right)_{a}\left(\nabla_{\hat{g}}\right)_{b} \\
& +\nabla_{\hat{g}} H_{g} \cdot \nabla_{\hat{g}} \varphi+\left(F-\frac{R_{g}}{3}\right) \frac{\partial \varphi}{\partial n_{g}} . \\
T_{g}= & -\frac{1}{12} \frac{\partial R_{g}}{\partial n_{g}}+\frac{1}{2} R_{g} H_{g}-<G_{g}, L_{g}>+3 H_{g}^{3}-\frac{1}{3} \operatorname{Tr}\left(L^{3}\right)+\Delta_{\hat{g}} H_{g},
\end{aligned}
$$

where $\varphi$ is any smooth function on $M, \hat{g}$ is the metric induced by $g$ on $\partial M, L_{g}=\left(L_{g}\right)_{a b}=-\frac{1}{2} \frac{\partial g_{a b}}{\partial n_{g}}$ is the second fundamental form of $\partial M, H_{g}=$ $\frac{1}{3} \operatorname{tr}\left(L_{g}\right)=\frac{1}{3} g^{a b} L_{a b}\left(g^{a, b}\right.$ are the entries of the inverse $g^{-1}$ of the metric $\left.g\right)$ is the mean curvature of $\partial M, R_{b c d}^{k}$ is the Riemann curvature tensor $F=$ $R_{n a n}^{a}, R_{a b c d}=g_{a k} R_{b c d}^{k}\left(g_{a, k}\right.$ are the entries of the metric $\left.g\right)$ and $\left\langle G_{g}, L_{g}\right\rangle=$ $R_{a n b n}\left(L_{g}\right)_{a b}$.

On the other hand, as the Laplace-Beltrami operator and the Neumann operator govern the transformation laws of the Gauss curvature and the geodesic curvature on compact surfaces with boundary under conformal change of metric, we have that the couple $\left(P_{g}^{4}, P_{g}^{3}\right)$ does the same for $\left(Q_{g}, T_{g}\right)$ on compact four dimensional smooth Riemannian manifolds with boundary. In fact, after a conformal change of metric $g_{u}=e^{2 u} g$, we have that

$$
\left\{\begin{array} { l } 
{ P _ { g } ^ { 4 } u = e ^ { - 4 u } P _ { g } ^ { 4 } ; } \\
{ P _ { g } ^ { 3 } u = e ^ { - 3 u } P _ { g } ^ { 3 } ; }
\end{array} \text { and } \quad \left\{\begin{array}{l}
P_{g}^{4}+2 Q_{g}=2 Q_{g_{u}} e^{4 u} \text { in } M \\
P_{g}^{3}+T_{g}=T_{g_{u}} e^{3 u} \text { on } \partial M .
\end{array}\right.\right.
$$

Apart from this analogy, we have also an extension of the Gauss-Bonnet formula (1.1) which is known as the Gauss-Bonnet-Chern formula

$$
\int_{M}\left(Q_{g}+\frac{\left|W_{g}\right|^{2}}{8}\right) d V_{g}+\int_{\partial M}(T+Z) d S_{g}=4 \pi^{2} \chi(M),
$$

where $W_{g}$ denote the Weyl tensor of $(M, g)$ and $Z d S_{g}$ (for the definition of $Z$, see [6]) are pointwise conformally invariant. Moreover, it turns out that $Z$ vanishes when the boundary is totally geodesic (by totally geodesic we mean that the boundary $\partial M$ is umbilic and minimal). Setting

$$
\kappa_{P_{g}^{4}}=\int_{M} Q_{g} d V_{g}, \quad \kappa_{P_{g}^{3}}=\int_{\partial M} T_{g} d S_{g}
$$

we have that, thanks to (1.2), and to the fact that $W_{g} d V_{g}$ and $Z d S_{g}$ are pointwise conformally invariant, $\kappa_{P_{g}^{4}}+\kappa_{P_{g}^{3}}$ is conformally invariant and will 
be denoted by

$$
\kappa_{\left(P^{4}, P^{3}\right)}=\kappa_{P_{g}^{4}}+\kappa_{P_{g}^{3}} .
$$

To mention some geometric applications, we discuss two results which can be found in the survey [12]. The first one is a rigidity-type result, more precisely it says that if $(M, g)$ has a constant positive scalar curvature and $\partial M$ has zero second fundamental form, then $\kappa_{\left(P^{4}, P^{3}\right)} \leq 4 \pi^{2}$; and equality holds if and only if $(M, \partial M)$ is conformally equivalent to the upper hemisphere $\left(S_{+}^{4}, S^{3}\right)$. The second one is a classification of the pairs $(M, \partial M)$ with $Q=0$, and $T=0$. Indeed it says that, if $(M, \partial M)$ is locally conformally flat with umbilic boundary $\partial M, Q=0, T=0, Y(g)>0$ (where $Y(g)=\inf \left\langle L_{c} u, u\right\rangle$ where the infimum is taken over all metrics conformal to $g$ with the same volume as $g$ and zero mean curvature and $L_{c}=-6 \Delta_{g}+R$ is the conformal Laplacian) and $\chi(M)=0$, then either $(M, \partial M)=\left(S^{1} \times S_{+}^{3}, S^{1} \times S^{2}\right)$, or $(M, \partial M)=\left(\mathrm{I} \times S^{3}, \partial \mathrm{I} \times S^{3}\right)$, where $\mathrm{I}$ is an interval.

A natural question to ask is whether every compact four-dimensional smooth Riemannian manifold with smooth boundary $(M, g)$ carries a conformal metric $g_{u}$ for which the corresponding $Q$-curvature $Q_{g_{u}}$ is constant, the corresponding $T$-curvature $T_{g_{u}}$ is zero and such that $\left(M, g_{u}\right)$ has minimal boundary. A related question was posed for the Yamabe problem, see [16].

Our goal in this paper is to give a positive answer to this question under generic conformally invariant assumptions. Writing $g_{u}=e^{2 u} g$, the problem is equivalent to solving the following BVP:

$$
\begin{cases}P_{g}^{4} u+2 Q_{g}=2 \bar{Q} e^{4 u} & \text { in } M, \\ P_{g}^{3} u+T_{g}=0 & \text { on } \partial M, \\ \frac{\partial u}{\partial n_{g}}-H_{g} u=0 & \text { on } \partial M,\end{cases}
$$

where $\bar{Q}$ is a fixed real number and $\frac{\partial}{\partial n_{g}}$ is the inward normal derivative with respect to $g$.

Due to a result by Escobar [16] and to the fact that we are interested to solve the problem under conformally invariant assumptions, it is not restrictive to assume $H_{g}=0$, since this can be always obtained through a conformal transformation of the background metric. Thus we are led to solve 
the following BVP with the Neumann homogeneous boundary condition:

$$
\begin{cases}P_{g}^{4} u+2 Q_{g}=2 \bar{Q} e^{4 u} & \text { in } M, \\ P_{g}^{3} u+T_{g}=0 & \text { on } \partial M, \\ \frac{\partial u}{\partial n_{g}}=0 & \text { on } \partial M .\end{cases}
$$

Defining $H_{\partial / \partial n}$ as

$$
H_{\partial / \partial n}=\left\{u \in H^{2}(M): \frac{\partial u}{\partial n_{g}}=0\right\},
$$

and $P_{g}^{4,3}$ as follows, for every $u, v \in H_{\partial / \partial n}$,

$$
\begin{aligned}
\left\langle P_{g}^{4,3} u, v\right\rangle_{L^{2}(M)}= & \int_{M}\left(\Delta_{g} u \Delta_{g} v+\frac{2}{3} R_{g} \nabla_{g} u \nabla_{g} v\right) d V_{g} \\
& -2 \int_{M} \operatorname{Ric}_{g}\left(\nabla_{g} u, \nabla_{g} v\right) d V_{g}-2 \int_{\partial M} L_{g}\left(\nabla_{\hat{g}} u, \nabla_{\hat{g}} v\right) d S_{g},
\end{aligned}
$$

we have that by the regularity result in Proposition 2.4 below, critical points of the functional

$$
\begin{aligned}
\mathrm{II}(u)= & \left\langle P^{4,3} u, u\right\rangle_{L^{2}(M)}+4 \int_{M} Q_{g} u d V_{g}+4 \int_{\partial M} T_{g} u d S_{g} \\
& -\kappa_{\left(P^{4}, P^{3}\right)} \log \int_{M} e^{4 u} d V_{g} ; \quad u \in H_{\partial / \partial n},
\end{aligned}
$$

which are weak solutions of (1.4) are also smooth and hence strong solutions.

To the best of our knowledge, the first existence result for problem (1.4) has been obtained by Chang and Qing, see [7] under the assumptions that $P_{g}^{4,3}$ is non-negative, Ker $P_{g}^{4,3} \simeq \mathbb{R}$ and $\kappa_{\left(P^{4}, P^{3}\right)}<4 \pi^{2}$.

In the case of closed four-dimensional Riemannian manifold $M$, it is well-known that the $Q$-curvature equation is intimately related to a fully non-linear PDE (the $\sigma_{2}$-equation), see $[10,11]$. A study of the latter PDE has given important geometric applications of the $Q$-curvature. In [10,11], it is proven that if the underlying Riemannian manifold has a conformal metric of positive constant scalar curvature and $\int_{M} Q_{g} d V_{g}>0$, then its first Betti number vanishes. Moreover, up to a conformal metric it has positive Ricci tensor, and hence $M$ has a finite fundamental group. Furthermore, if the quantitative assumption $\int_{M} Q_{g} d V_{g}>\frac{1}{8} \int_{M}\left|W_{g}\right|^{2} d V_{g}$ holds, then $M$ must be diffeomorphic to the four-sphere or to the projective space. 
For the case where $M$ has a boundary, Chen [13] has studied an analogue of the $\sigma_{2}$-equation, which turns out to be a fully non-linear BVP. Among others results, she obtained that if the Yamabe invariant $Y(M, \partial M,[g]$ ) (for the definition, see [13]) and $\kappa_{\left(P^{4}, P^{3}\right)}$ are both positive and $M$ umbilic, then there exists a metric $g_{u}$ in the conformal class of $g$ such that $\sigma_{2}\left(A_{g_{u}}\right)$ (the second symmetric function of the Shouten tensor $A_{g_{u}}$ ) is a positive constant $H_{g_{u}}=0$. Furthermore, $g_{u}$ can be taken so that the Ricci curvature Ric $_{g_{u}}$ is positive, hence $M$ has a finite fundamental group.

Here we extend the uniformization result of Chang and Qing mentioned above, namely we prove existence of solutions to (1.4) under more general conditions. Precisely we obtain the following result, which is the main theorem in this paper:

Theorem 1.1. Suppose $\operatorname{Ker} P_{g}^{4,3} \simeq \mathbb{R}$. Then assuming $\kappa_{\left(P^{4}, P^{3}\right)} \neq k 4 \pi^{2}$ for $k=1,2, \ldots$, we have that $(M, g)$ admits a conformal metric with constant $Q$-curvature, zero $T$-curvature and zero mean curvature.

Remark 1.2. (a) Our assumptions are conformally invariant and generic, so the result applies to a large class of compact four-dimensional manifolds with boundary.

(b) From the Gauss-Bonnet-Chern formula, see (1.2), we have that Theorem 1.1 does NOT cover the case of locally conformally flat manifolds with totally geodesic boundary and positive integer Euler-Poincaré characteristic.

(c) For the boundary Yamabe problem in low dimension (less than 5), existence of solutions was obtained only under the assumption of local conformal flatness of the manifold and umbilicity of the boundary. However, in our theorem, we point out that no umbilicity condition for the boundary $\partial M$ and no flatness condition for $M$ are assumed.

Our assumptions include the following two situations:

$\kappa_{\left(P^{4}, P^{3}\right)}<4 \pi^{2}$ and (or) $P_{g}^{4,3}$ possesses $\bar{k}$ negative eigenvalues

(counted with multiplicity),

$\kappa_{\left(P^{4}, P^{3}\right)} \in\left(4 k \pi^{2}, 4(k+1) \pi^{2}\right), \quad$ for some $k \in \mathbb{N}^{*} \quad$ and (or) $P_{g}^{4,3}$ possesses $\bar{k}$ negative eigenvalues (counted with multiplicity).

Remark 1.3. Case (1.5) includes the condition $(\bar{k}=0)$ under which Chang and Qing have proved existence of solutions to (1.4), hence will not be 
considered here. However, due to a Moser-Trudinger-type inequality (see Proposition 2.7 below) it can be achieved using direct method of calculus of variations.

In order to simplify the exposition, we will give the proof of Theorem 1.1 in the case where we are in situation (1.6) and $\bar{k}=0$ (namely $P_{g}^{4,3}$ is non-negative). At the end of Section 4, a discussion to settle the general case (1.6) and also case (1.5) is made.

To prove Theorem 1.1, we look for critical points of II. Unless $\kappa_{\left(P^{4}, P^{3}\right)}<$ $4 \pi^{2}$ and $\bar{k}=0$, this Euler-Lagrange functional is unbounded from above and below (see Section 4), so it is necessary to find extremals which are possibly saddle points. To do this, we will use a min-max method: by classical arguments in critical point theory, the scheme yields a Palais-Smale sequence, namely a sequence $\left(u_{l}\right)_{l} \in H_{\partial / \partial n}$ satisfying the following properties

$$
\mathrm{II}\left(u_{l}\right) \longrightarrow c \in \mathbb{R} ; \quad \mathrm{II}^{\prime}\left(u_{l}\right) \longrightarrow 0 \text { as } l \longrightarrow+\infty \text {. }
$$

Then, as is usually done in min-max theory, to recover existence one should prove that the so-called Palais-Smale condition holds, namely that every Palais-Smale sequence has a converging subsequence or a similar compactness criterion. Since we do not know if the Palais-Smale condition holds, we will employ Struwe's monotonicity method, see [20], also used in [14] and [18]. The latter yields existence of solutions for arbitrary small perturbations of the given equation, so to consider the original problem one is led to study compactness of solutions to perturbations of (1.4). Precisely, we consider

$$
\begin{cases}P_{g}^{4} u_{l}+2 Q_{l}=2 \bar{Q}_{l} e^{4 u} & \text { in } M, \\ P_{g}^{3} u+T_{l}=0 & \text { on } \partial M, \\ \frac{\partial u_{l}}{\partial n_{g}}=0 & \text { on } \partial M,\end{cases}
$$

where

$$
\bar{Q}_{l} \longrightarrow \bar{Q}_{0}>0 \text { in } C^{2}(M) ; \quad Q_{l} \longrightarrow Q_{0} \text { in } C^{2}(M) ; \quad T_{l} \longrightarrow T_{0} \text { in } C^{2}(\partial M) .
$$

Adopting the standard terminology in geometric analysis, we say that a sequence $\left(u_{l}\right)$ of solutions to (1.7) blows up if the following holds:

$$
\text { there exist } x_{l} \in M \text { such that } u_{l}\left(x_{l}\right) \longrightarrow+\infty \text { as } l \longrightarrow+\infty \text {, }
$$

and we prove the following compactness result. 
Theorem 1.4. Suppose $\operatorname{Ker} P_{g}^{4,3} \simeq \mathbb{R}$ and that $\left(u_{l}\right)$ is a sequence of solutions to (1.7) with $\bar{Q}_{l}, Q_{l}$ and $T_{l}$ satisfying (1.8). Assuming that $\left(u_{l}\right)_{l}$ blows up (in the sense of (1.9)) and

$$
\int_{M} Q_{0} d V_{g}+\int_{\partial M} T_{0} d S_{g}+o_{l}(1)=\int_{M} \bar{Q}_{l} e^{4 u_{l}} d V_{g}
$$

then there exists $N \in \mathbb{N} \backslash\{0\}$ such that

$$
\int_{M} Q_{0} d V_{g}+\int_{\partial M} T_{0} d S_{g}=4 N \pi^{2}
$$

From this, we derive a corollary which will be used to ensure compactness of some solutions to a sequence of approximate BVPs produced by the topological argument combined with Struwe's monotonicity argument. Its proof is a trivial application of Theorem 1.4 and Proposition 2.4 below.

Corollary 1.5. Suppose $\operatorname{Ker} P_{g}^{4,3} \simeq \mathbb{R}$.

(a) Let $\left(u_{l}\right)$ be a sequence of solutions to (1.7) with $\bar{Q}_{l}, Q_{l}$ and $T_{l}$ satisfying (1.8). Assume also that

$$
\int_{M} Q_{0} d V_{g}+\int_{\partial M} T_{0} d S_{g}+o_{l}(1)=\int_{M} \bar{Q}_{l} e^{4 u_{l}} d V_{g}
$$

and

$$
k_{0}=\int_{M} Q_{0} d V_{g}+\int_{\partial M} T_{0} d S_{g} \neq 4 k \pi^{2}, \quad k=1,2,3, \ldots,
$$

then $\left(u_{l}\right)_{l}$ is bounded in $C^{4+\alpha}(M)$ for any $\alpha \in(0,1)$.

(b) Let $\left(u_{l}\right)$ be a sequence of solutions to (1.4) for a fixed value of the constant $\bar{Q}$. Assume also that

$$
\kappa_{\left(P^{4}, P^{3}\right)} \neq 4 k \pi^{2}
$$

Then $\left(u_{l}\right)_{l}$ is bounded in $C^{m}(M)$ for every positive integer $m$.

(c) Let $\left(u_{\rho_{k}}\right) \rho_{k} \rightarrow 1$ be a family of solutions to (1.4) with $T_{g}$ replaced by $\rho_{k} T_{g}, Q_{g}$ by $\rho_{k} Q_{g}$ and $\bar{Q}$ by $\rho_{k} \bar{Q}$ for a fixed value of the constant $\bar{Q}$. Assume also that

$$
\kappa_{\left(P^{4}, P^{3}\right)} \neq 4 k \pi^{2} .
$$

Then $\left(u_{\rho_{k}}\right)_{k}$ is bounded in $C^{m}(M)$ for every positive integer $m$. 
(d) If $\kappa_{\left(P^{4}, P^{3}\right)} \neq 4 k \pi^{2}, k=1,2,3, \ldots$, then the set of metrics conformal to $g$ with constant $Q$-curvature, zero $T$-curvature, zero mean curvature and of unit interior volume is compact in $C^{m}(M)$ for every positive integer $m$.

We are going to describe our method to prove the above results. Since the proof of Theorem 1.1 relies on the compactness result of Theorem 1.4 (see Corollary 1.5), it is convenient to discuss first the latter. We use a strategy related to that in [15], but in our case we have to consider possible blow-ups at the boundary. In [18], a variant of this method was used, which relies strongly on the Green representation formula, transforming (1.4) into an integral equation. Here we will employ a similar strategy since, for the BVP one can prove the existence of a Green representation formula as well (using the method of the parametrix) with the difference that we have a boundary term, see Lemma 2.3. We consider the same scaling as in [15] and [18]. When we deal with the situation of interior blow-up points, we adopt the same strategy as in [18] to get that the limit function $V_{0}$ which describes the profile near the blow-up point satisfies the following conformally invariant integral equation

$$
\tilde{V}_{0}(x)=\int_{\mathbb{R}^{4}} \frac{3}{4 \pi^{2}} \log \left(\frac{|z|}{|x-z|}\right) e^{4 \tilde{V}_{0}(z)} d z-\frac{1}{4} \log (3) .
$$

Hence using the same argument as in [18], based on a classification result of $\mathrm{Xu}$ [22], we deduce that $V_{0}$ is a standard bubble and the local volume is $8 \pi^{2}$. On the other hand, when the blow-up phenomenon happens at the boundary, we obtain that the limiting function satisfies the integral equation on the upper half-space $\mathbb{R}_{+}^{4}$

$$
V_{0}(x)=\int_{\mathbb{R}_{+}^{4}} \frac{3}{4 \pi^{2}}\left(\log \frac{|z|}{|x-z|}+\log \frac{|z|}{|x-\bar{z}|}\right) e^{4 V_{0}(z)} d z-\frac{1}{4} \log (3) .
$$

So from this, we are able to deduce that the normal derivative of $V_{0}$ vanishes. Thus using Alexandrov reflection principle, we infer that the even reflection across $\partial \mathbb{R}_{+}^{4} \bar{V}_{0}$ of $V_{0}$ solves the conformally invariant integral equation on the entire space $\mathbb{R}^{4}$ as in (1.11).

So we can use the classification result of $\mathrm{Xu}$ (mentioned above) to deduce that $\bar{V}_{0}$ is a standard bubble and that the local volume associated is $8 \pi^{2}$. Hence we find that the profile near such blow-up point (boundary) is half of a standard bubble and that the local volume associated is $4 \pi^{2}$. At this stage, to finish we argue as in [18] to show that the residual volume tends to 
zero and obtain quantization. We point out that, by the above discussion, the volume of an interior blow-up is double with respect to the one at the boundary.

Now having this compactness result, we can describe the proof of Theorem 1.1 assuming (1.6) and that $P_{g}^{4,3}$ is non-negative. In [14] and [18], the existence theorem was proved considering the formal barycenters of the manifold $M$ (which we will describe below), together with the differences with respect to the present case, which are mainly two. The arguments in [14] (similarly in [18]) can be summarized as follows. First of all from $\kappa_{P^{4}} \in\left(k 8 \pi^{2},(k+1) 8 \pi^{2}\right)$ and considerations coming from an improvement of Moser-Trudinger inequality, it follows that if $\mathrm{II}(u)$ attains large negative values, then $e^{4 u}$ has to concentrate near at most $k$ points of $M$. This means that, if we normalize $u$ so that $\int_{M} e^{4 u} d V_{g}=1$, then naively $e^{4 u} \simeq \sum_{i=1}^{k} t_{i} \delta_{x_{i}}, x_{i} \in M, t_{i} \geq 0, \sum_{i=1}^{k} t_{i}=1$. Such a family of convex combination of Dirac deltas are called formal barycenters of $M$ of order $k$, see Section 2 in [14], and will be denoted by $M_{k}$. With a further analysis (see Proposition 3.1 in [14]), it is possible to show that the sublevel $\{\mathrm{II}<-L\}$ for large $L$ has the same homology as $M_{k}$. Using this fact and the noncontractibility of $M_{k}$ (which is a crucial ingredient), existence of solutions was found.

The differences with the present case are the fact that $M_{k}$ might be contractible and also boundary concentration can appear, hence new ideas are needed. We first perform a more refined study of how big can be the number of possible boundary and interior blow-up points for the conformal volume $e^{4 u}, u \in\left\{v \in H_{\partial / \partial n} \int_{M} e^{4 v} d V_{g}=1 ; \operatorname{II}(v) \leq-L\right\}$ with $L$ large enough. In doing this, we first prove a Moser-Trudinger-type inequality using blow-up analysis techniques, see Proposition 2.7. Next we derive an improvement of it by following basically the same arguments as in $[15,18]$ and taking into account that far from the boundary we can use the MoserTrudinger inequality for closed manifolds (see Lemma 2.1). As a corollary, we derive that if $k$ is as in (1.6) and if $\operatorname{II}\left(u_{l}\right) \rightarrow-\infty$ along a sequence $u_{l}$ with $\int_{M} e^{4 u_{l}} d V_{g}=1$, then $e^{4 u_{l}}$ has to concentrate near at most $h$ interior points and $l$ boundary points with $2 h+l \leq k$ and $e^{4 u_{l}} \simeq \sigma=\sum_{i=1}^{h} t_{i} \delta_{x_{i}}+$ $\sum_{i=1}^{l} s_{i} \delta_{y_{i}}, t_{i} \geq 0, \sum_{i=1}^{h} t_{i}+\sum_{i=1}^{l} s_{i}=1 ; x_{i} \in \operatorname{int}(M), y_{i} \in \partial M$. Therefore, instead of $M_{k}$, it is natural to consider the barycentric set $\left(M_{\partial}\right)_{k}$ (for the definition, see Section 2) which is a good candidate for describing the homology of large negative sublevels of II. In order to do this, one needs to map (non-trivially) the large negative sublevels into $\left(M_{\partial}\right)_{k}$, and to do the opposite, namely to map $\left(M_{\partial}\right)_{k}$ (non-trivially) onto low sublevels of II. If the composition of these two maps is homotopic to the identity, we derive the 
topology of the low sublevels of II, in terms of the number of concentration points of the conformal volume $e^{4 u}$. To find the projection onto $\left(M_{\partial}\right)_{k}$, we can use some of the arguments in [14], but with evident differences, because of the presence of the boundary. Taking advantage of the fact that the functions we are dealing with have zero normal derivatives, we use a doubling argument, which consists of constructing a new $C^{1}$ manifold $D M$, and using the Alexandrov reflection principle (see Proposition 4.8 below). We then use some suitable test functions to find the desired homotopy equivalence.

Using the Mayers-Vietoris theorem, one can prove that $\left(M_{\partial}\right)_{k}$ is noncontractible, see Proposition 4.6, and define a min-max scheme for a perturbed functional $\mathrm{II}_{\rho}, \rho$ close to 1 , finding a P-S sequence to some levels $c_{\rho}$. Applying the monotonicity procedure of Struwe, we can show existence of critical points of $\mathrm{II}_{\rho}$ for a.e $\rho$, and we reduce ourselves to the assumptions of Theorem 1.5.

The structure of the paper is the following. In Section 2 we collect some notations and give some preliminaries like the existence of the Green function for $\left(P_{g}^{4}, P_{g}^{3}\right)$ with homogeneous Neumann conditions, a Moser-Trudingertype inequality and a regularity result for BVPs of the type of (1.4). In Section 3 we prove Theorem 1.4 (from which the proof of Corollary 1.5 becomes a trivial application). In Section 4 we finally prove Theorem 1.1. The latter section is divided into four subsections. The first one concerns an improvement of the Moser-Trudinger-type inequality and applications. The second deals with the existence of a non-trivial projection from negative sublevels of II onto $\left(M_{\partial}\right)_{k}$. The third is concerned about mapping $\left(M_{\partial}\right)_{k}$ into negative sublevels, and the last deals with the min-max scheme.

\section{Notation and preliminaries}

In this brief section, we collect some useful notations, state a lemma giving the existence of the Green function of the operator $\left(P_{g}^{4}, P_{g}^{3}\right)$ with its asymptotics near the singularity and an analog of the well-known Moser-Trudinger inequality for the operator $P_{g}^{4,3}$ when it is non-negative.

In the following, $B_{p}(r)$ stands for the metric ball of radius $r$ and center $p, B_{p}^{+}(r)=B_{p}(r) \cap M$ if $p \in \partial M$. Sometimes we use $B_{p}^{+}(r)$ to denote $B_{p}(r) \cap M$ even if $p \notin \partial M$. For a given integer $n$, we denote by $\mathbb{R}^{n}$ the standard $n$-dimensional Euclidean space and $\mathbb{R}_{+}^{n}=\left\{x=\left(x_{1}, \ldots, x_{n}\right) \in \mathbb{R}^{n}\right.$ : $\left.x_{n} \geq 0\right\}$. In the sequel, $B^{x}(r)$ will stand for the Euclidean ball of center $x$ and radius $r, B_{+}^{x}(r)=B^{x}(r) \cap \mathbb{R}_{+}^{4}$ if $x \in \partial \mathbb{R}_{+}^{4}$. We use also $B_{+}^{x}(r)$ to denote $B^{x}(r) \cap \mathbb{R}_{+}^{4}$ even if $x \notin \partial \mathbb{R}_{+}^{4}$. We denote by $d_{g}(x, y)$ the metric distance 
between two points $x$ and $y$ of $M . H^{2}(M)$ stands for the usual Sobolev space of functions on $M$ which are of class $H^{2}$ in each coordinate system. Large positive constants are always denoted by $C$, and the value of $C$ is allowed to vary from formula to formula and also within the same line. $M^{2}$ stands for the Cartesian product $M \times M$, while $\operatorname{Diag}(M)$ is the diagonal of $M^{2}$. Given a function $u \in L^{1}(M), \bar{u}$ denotes its average on $M$, that is, $\bar{u}=\left(\operatorname{Vol}_{g}(M)\right)^{-1} \int_{M} u(x) d V_{g}(x)$, where $\operatorname{Vol}_{g}(M)=\int_{M} d V_{g}$.

Given two subsets $S_{1}, S_{2}$ of $M$, we set

$$
d_{g}\left(S_{1}, S_{2}\right)=\inf \left\{d_{g}(x, y) x \in S_{1}, y \in S_{2}\right\}
$$

and for $x \in M$ fixed we define

$$
d_{g}\left(x, S_{1}\right)=\inf \left\{d_{g}(x, y) y \in S_{1}\right\}
$$

$\operatorname{int}(M)$ denotes the topological interior of $M$.

$\mathbb{N}$ denotes the set of non-negative integers.

$\mathbb{N}^{*}$ stands for the set of positive integers.

$A_{l}=o_{l}(1)$ means that $A_{l} \rightarrow 0$ as the integer $l \rightarrow+\infty$.

$A_{\epsilon}=o_{\epsilon}(1)$ means that $A_{\epsilon} \rightarrow 0$ as the real number $\epsilon \rightarrow 0$.

$A_{\delta}=o_{\delta}(1)$ means that $A_{\delta} \rightarrow 0$ as the real number $\delta \rightarrow 0$.

$A_{l}=O\left(B_{l}\right)$ means that $A_{l} \leq C B_{l}$ for some fixed constant $C$.

$\operatorname{inj}_{g}(M)$ stands for the injectivity radius of $M$.

$d V_{g}$ denotes the Riemannian measure associated to the metric $g$.

$d S_{g}$ stands for the Riemannian measure associated to the metric $\hat{g}$ induced by $g$ on $\partial M$.

$d \sigma_{g}$ stands for the surface measure on boundary of balls, $|\cdot|_{g}$ stands for the norm associated to $g$.

$f=f(a, b, c, \ldots)$ means that $f$ is a quantity which depends only on $a, b, c, \ldots$ Given an operator $P$ acting on functions $u(x, y)$ defined on $M^{2}, P_{y}$ means the action of $P$ with respect to the variable $y \in M$.

Given a metric $g$ on $M,|g(x)|, x \in M$, stands for determinant of the matrix with entries $g_{i, j}(x)$, where $g_{i, j}(x)$ are the components of $g(x)$, in some system of coordinates.

For $\epsilon>0$, we set

$$
(\partial M)^{\epsilon}=\left\{x \in M d_{g}(x, \partial M) \leq \epsilon\right\} .
$$

We also set

$$
\tilde{k}=\left[\frac{k}{2}\right],
$$


where $\left[\frac{k}{2}\right]$ stands for the integer part of $\frac{k}{2}$.

Now given $\delta>0$ a small positive constant, we set

$$
M_{\delta}=M \backslash \partial M \times[0, \delta] .
$$

Next we let $(\partial M)_{k}$ denote the family of formal sums

$$
(\partial M)_{k}=\left\{\sum_{i=1}^{k} t_{i} \delta_{x_{i}}, t_{i} \geq 0, \sum_{i=1}^{k} t_{i}=1 ; x_{i} \in \partial M\right\}
$$

It is known in the literature as the formal set of barycenters relative to $\partial M$ of order $k$. We recall that $(\partial M)_{k}$ is a stratified set, namely a union of sets of different dimensions with maximum one equal to $4 k-1$.

Now given $h \in \mathbb{N}, l \in \mathbb{N}$ such that $h \leq \tilde{k}, l \leq k$ and $2 h+l \leq k$, we define $M_{h, l}$ as follows:

$$
\begin{aligned}
M_{h, l}=\{ & \sum_{i=1}^{h} t_{i} \delta_{x_{i}}+\sum_{i=1}^{l} s_{i} \delta_{y_{i}}, t_{i} \geq 0, \sum_{i=1}^{h} t_{i} \\
& \left.+\sum_{i=1}^{l} s_{i}=1 ; x_{i} \in \operatorname{int}(M), y_{i} \in \partial M\right\}
\end{aligned}
$$

We set also

$$
\left(M_{\partial}\right)_{k}=\cup_{h, l} M_{h, l} .
$$

$\left(M_{\partial}\right)_{k}$ will be endowed with the weak topology of distributions. To carry out some computations, we will use on $\left(M_{\partial}\right)_{k}$ the metric given by $C^{1}(M)^{*}$, which induces the same topology, and which will be denoted by $d_{M}(\cdot, \cdot)$.

Now let us introduce some further definitions.

Given $\sigma \in\left(M_{\partial}\right)_{k}, \sigma=\sum_{i=1}^{h} t_{i} \delta_{x_{i}}+\sum_{i=1}^{l} s_{i} \delta_{y_{i}}$ with $x_{i} \in \operatorname{int}(M), y_{i} \in$ $\partial M$ and $2 h+l \leq k$, we set

$$
\sigma_{\text {int }}=\sum_{i=1}^{h} t_{i} \delta_{x_{i}}
$$

and

$$
\sigma_{\mathrm{bdry}}=\sum_{i=1}^{l} s_{i} \delta_{y_{i}}
$$


Next for $\varphi \in C^{1}(M)$ and $\sigma=\sigma_{\text {int }}+\sigma_{\text {bdry }} \in\left(M_{\partial}\right)_{k}$, we denote the action of $\sigma$ on $\varphi$ as

$$
<\sigma, \varphi>=\sum_{i=1}^{h} t_{i} \varphi\left(x_{i}\right)+\sum_{i=1}^{l} s_{i} \varphi\left(y_{i}\right)
$$

where $\sigma_{\text {int }}=\sum_{i=1}^{h} t_{i} \delta_{x_{i}}$ and $\sigma_{\text {bdry }}=\sum_{i=1}^{l} s_{i} \delta_{y_{i}}$.

Next if $f$ is a non-negative $L^{1}$ function on $M$ with $\int_{M} f d V_{g}=1$ and $S \subset\left(M_{\partial}\right)_{k}$, we define the distance of $f$ from $S$ as follows:

$$
d_{M}(f, S)=\inf _{\sigma \in S} d_{M}(f, \sigma) .
$$

To finish the part of the notations, we define

$$
B_{\epsilon, k}=\left\{\sigma \in\left(M_{\partial}\right)_{k}: d_{M}\left(\sigma,(\partial M)_{k}\right) \leq \epsilon\right\}
$$

Now we recall the following Moser-Trudinger-type inequality for closed four-dimensional smooth Riemannian manifolds. Its proof can be found in $[9$, Lemma 1.6].

Lemma 2.1. Let $(N, g)$ be a four dimensional compact closed manifold. Assume that $P_{g}^{4}$ is non-negative and that $\operatorname{KerP}_{g}^{4} \simeq \mathbb{R}$, then there exists a constant $C=C(N)>0$ such that

$$
\int_{N} e^{32 \pi^{2} u^{2}} d V_{g} \leq C \quad \text { for all } u \in H^{2}(N) \text { such that }\left\langle P_{g}^{4} u, u\right\rangle_{L^{2}(N)}=1
$$

and hence

$$
\log \int_{N} e^{4(u-\bar{u})} \leq C+\frac{1}{8 \pi^{2}}\left\langle P_{g}^{2} u, u\right\rangle_{L^{2}(N)}
$$

Remark 2.1. We remark that the constant $C=C(N)$ in the lemma above is an increasing function of $\operatorname{Vol}_{g}(N)$. This fact will be used in the proof of Lemma 4.1 below.

Next we give a Lemma which can be found in [7, Proposition A.1].

Lemma 2.2. There exists an extension of $(M, g)$ into $(\tilde{M}, \tilde{g})$ which is a closed smooth four-dimensional Riemannian manifold such that

1) $M$ is an open submanifold of $\tilde{M}$,

2) $\tilde{g} \mid M=g$, 
3) In $\tilde{M}$, $\partial M$ has a nice tubular neighborhood $T$ of width $\delta_{0}$ such that, for any $x \in T \cap M$, there exists a unique $\bar{x} \in T \backslash M$ with $d_{\bar{g}}(x, \partial M)=$ $d_{\bar{g}}(\bar{x}, \partial M)$, and for $x \in \partial M, x=\bar{x}$, where $d_{\bar{g}}$ denotes the Riemannian distance associated to $\bar{g}$.

Now we state a proposition which asserts the existence of the Green function of $\left(P_{g}^{4}, P_{g}^{3}\right)$ with homogeneous Neumann condition. Moreover, we give its asymptotics near the singularities.

Proposition 2.3. Assume that $\operatorname{Ker} P_{g}^{4,3} \simeq \mathbb{R}$, then the Green function $G(x$, y) of $\left(P_{g}^{4}, P_{g}^{3}\right)$ exists in the following sense:

a) For all functions $u \in C^{2}(M), \frac{\partial u}{\partial n_{g}}=0$, we have

$$
u(x)-\bar{u}=\int_{M} G(x, y) P_{g}^{4} u(y) d V_{g}(y)+2 \int_{\partial M} G\left(x, y^{\prime}\right) P_{g}^{3} u(t) d S_{g}\left(y^{\prime}\right), \quad x \in M
$$

b)

$$
G(x, y)=H(x, y)+K(x, y)
$$

is smooth on $M^{2} \backslash \operatorname{Diag}\left(M^{2}\right), K$ extends to a $C^{2+\alpha}$ function on $M^{2}$ and

$$
H(x, y)= \begin{cases}\frac{1}{8 \pi^{2}} f(r) \log \frac{1}{r} & \text { if } B_{\delta}(x) \cap \partial M=\emptyset \\ \frac{1}{8 \pi^{2}} f(r)\left(\log \frac{1}{r}+\log \frac{1}{\bar{r}}\right) & \text { otherwise }\end{cases}
$$

where $f(\cdot)=1$ in $\left[-\frac{\delta}{2}, \frac{\delta}{2}\right]$ and $f(\cdot) \in C_{0}^{\infty}(-\delta, \delta), \delta \leq \frac{1}{2} \min \left\{\delta_{1}, \delta_{2}\right\}, \delta_{1}$ is the injectivity radius of $M$ in $\tilde{M}$ and $\delta_{2}=\frac{\delta_{0}}{2}, r=d_{g}(x, y)$ and $\bar{r}=$ $d_{g}(x, \bar{y})$.

Proof. Let $x \in M$ be fixed, it is well known that in normal coordinate around $x$ the following holds

$$
|\tilde{g}(y)|=1+O\left(r^{2}\right) \quad \text { for } y \text { close to } x
$$

Now working in this normal coordinate system around $x$, we have that

$$
\left|P_{g}^{4} H(x, y)\right| \leq C r^{-2} \quad \text { for } r \leq C^{-1} \operatorname{inj}_{g}(M) .
$$

and

$$
\left|P_{g}^{3} H(x, y)\right| \leq C r^{-1} \quad \text { for } r \leq C^{-1} \operatorname{inj}_{g}(M) \text {. }
$$


On the other hand, by considering the expression

$$
\int_{M \backslash B_{x}(\epsilon)} H(x, y) P_{g}^{4} u(y) d V_{\tilde{g}}(y)-\int_{M \backslash B_{x}(\epsilon)} u(y) P_{g}^{4} H(x, y) u(y) d V_{\tilde{g}}(y),
$$

we have by integration by parts that

$$
\begin{aligned}
& \int_{M \backslash B_{x}(\epsilon)} H(x, y) P_{g}^{4} u(y) d V_{\tilde{g}}(y)-\int_{M \backslash B_{x}(\epsilon)} u(y) P_{g}^{4} H(x, y) u(y) d V_{g}(y) \\
& =-\int_{\partial B_{x}(\epsilon)} \frac{\partial \Delta_{\bar{g}, y}}{\partial n_{g}} H(x, y) u(y) d V_{g}(y)+2 \int_{\partial M} H\left(x, y^{\prime}\right) P_{g}^{3} d S_{\bar{g}}\left(y^{\prime}\right)+o_{\epsilon}(1) .
\end{aligned}
$$

Now by using the fact that close to $x$ in conformal normal coordinate $\Delta_{g}$ is close to $\Delta_{\mathbb{R}^{4}}$, we obtain by letting $\epsilon$ go to 0

$$
\begin{aligned}
u(x)= & \int_{M} H(x, y) P_{g}^{4} u(y) d V_{g}(y)-\int_{M} P_{g}^{4} H(x, y) u(y) d V_{\tilde{g}}(y) \\
& +2 \int_{\partial M} H\left(x, y^{\prime}\right) P_{g}^{3} u\left(y^{\prime}\right) d S_{g}\left(y^{\prime}\right) .
\end{aligned}
$$

Hence, for every $x \in M$, we obtain

$$
\begin{aligned}
u(x)= & \int_{M} H(x, y) P_{g}^{4} u(y) d V_{g}(y)-\int_{M} P_{g}^{4} H(x, y) u(y) d V_{g}(y) \\
& +2 \int_{\partial M} H\left(x, y^{\prime}\right) P_{g}^{3} u\left(y^{\prime}\right) d S_{g}\left(y^{\prime}\right) .
\end{aligned}
$$

Now we can apply the same method as in [2, Theorem 4.13] to construct parametrix for Green's function. We set

$$
G(x, y)=H(x, y)+\sum_{i=1}^{q} Z_{i}(x, y)+F(x, y)
$$

where $q>2$,

$$
Z_{i}(x, y)=\int_{M} \Gamma_{i}(x, \zeta) H(\zeta, y) d V_{g}(\zeta)
$$

and $\Gamma_{i}$ are defined inductively as follows:

$$
\Gamma_{i+1}(x, y)=\int_{M} \Gamma_{i}(x, \zeta) \Gamma(\zeta, y) d V_{g}(\zeta)
$$

with

$$
\Gamma_{1}(x, y)=\Gamma(x, y)=-P_{g, y}^{4} H(x, y)
$$


and $F$ being the solution of the equation

$$
\begin{cases}P_{g, y}^{4} F(x, y)=\Gamma_{k+1}(x, y)-\frac{1}{\operatorname{Vol}_{g}(M)} . & \text { in } M, \\ P_{g, y}^{3} F(x, y)=-P_{g, y}^{3} H(x, y) & \text { on } \partial M, \\ \frac{\partial F(x, y)}{\partial n_{g, y}}=0 & \text { on } \partial M .\end{cases}
$$

Now from (2.3) we have that $Z_{i}$ satisfies

$$
\begin{cases}P_{g, y}^{4} Z_{i}(x, y)=\Gamma_{i}(x, y)-\Gamma_{i+1}(x, y) & \text { in } M, \\ P_{g, y}^{3} Z_{i}(x, y)=0 & \text { on } \partial M, \\ \frac{\partial Z_{i}(x, y)}{\partial n_{g, y}}=0 & \text { on } \partial M .\end{cases}
$$

We observe that the following estimate holds for $\Gamma(x, y)$,

$$
|\Gamma(x, y)| \leq C r^{-2}
$$

hence by using the results in [2, Proposition 4.12], we obtain the following estimate for $\Gamma_{i}(x, y)$,

$$
\left|\Gamma_{i}(x, y)\right| \leq C r^{2 i-4}
$$

So arriving at this stage by still the same result in [2, Proposition 4.12], we have that $\Gamma_{q}(x, y)$ and $\Gamma_{q+1}(x, y)$ are continuous, hence using elliptic regularity we get $Z_{q}(x, y)$ and $F(x, y)$ are in $C^{3+\alpha}\left(M^{2}\right)$. The regularity in both the variables $x$ and $y$ can be deduced by the symmetry of $G$, which follows from the self-adjointness of $P_{g}^{4,3}$ and reasoning as in [2, Proposition 4.13]. Further from (2.4) we deduce that $\Gamma_{i} \in L^{p}$ with $\frac{1}{2}<p<2$ for all $i=1, \ldots, q-1$. Hence by using standard elliptic regularity, we infer that $Z_{i}(x, y) \in H^{4, p}$. So from the Sobolev embedding theorem and the fact that $\frac{1}{2}<p<2$, we get $Z_{i}(x, y) \in C^{2+\alpha}\left(M^{2}\right)$ for all $i=1, \ldots, q-1$ for some $\alpha$. Hence setting $K(x, y)=\sum_{i=1}^{q} Z_{i}(x, y)+F(x, y)$, the lemma is proved.

Next we give a regularity result corresponding to BVPs of the type of BVP (1.4) and high-order a priori estimates for sequences of solutions to BVP like (1.7) when they are bounded from above. 
Proposition 2.4. Let $u \in H_{\partial / \partial n}$ be a weak solution to

$$
\begin{cases}P_{g}^{4} u+f=\bar{f} e^{4 u} & \text { in } M, \\ P_{g}^{3} u=h & \text { on } \partial M,\end{cases}
$$

with $f \in C^{\infty}(M), h \in C^{\infty}(\partial M)$ and $\bar{f}$ a real constant. Then we have that $u \in C^{\infty}(M)$.

Let $u_{l} \in H_{\partial / \partial n}$ be a sequence of weak solutions to

$$
\begin{cases}P_{g}^{4} u_{l}+f_{l}=\bar{f}_{l} e^{4 u_{l}} & \text { in } M, \\ P_{g}^{3} u_{l}=h_{l} & \text { on } \partial M\end{cases}
$$

with $f_{l} \rightarrow f_{0}$ in $C^{k}(M), \bar{f}_{l} \rightarrow \bar{f}_{0}$ in $C^{k}(\partial M)$ and $h \rightarrow h_{0}$ in $C^{k}(\partial M)$ for some fixed $k \in \mathbb{N}^{*}$. Assuming $\sup _{M} u_{l} \leq C$, we have that

$$
\left\|u_{l}\right\|_{C^{k+3+\alpha}(M)} \leq C
$$

for any $\alpha \in(0,1)$.

Before making the proof of Proposition 2.4, we give some lemmas that will be needed. We first state a lemma which is a direct consequence of Lemma 2.2. Next we recall a lemma giving the existence of a Green function for the Paneitz operator on compact closed four-dimensional smooth Riemannian manifold.

Lemma 2.5. Adopting the same notations as in Lemma (2.2), we have that there exists a closed compact smooth four-dimensional submanifold $N$ of $(\tilde{M}, \tilde{g})$ such that $M \subset N$. Moreover, the following holds: if $\forall x \in N \backslash M$, there exists a unique $\bar{x} \in M \cap T$ such that

$$
d_{\tilde{g}}(x, \partial M)=d_{\tilde{g}}(\bar{x}, \partial M)
$$

As said above, we state a lemma giving the existence of the Green function for $P_{\tilde{g}}^{4}$. Its proof can be found in [9] or ([18] where it is done for every dimension).

Lemma 2.6. Suppose Ker $P_{\tilde{g}}^{4} \simeq \mathbb{R}$. Then the Green function $\tilde{G}(x, y)$ of $P_{\tilde{g}}^{4}$ exists in the following sense: 
a) For all functions $u \in C^{2}(N)$, we have

$$
u(x)-\bar{u}=\int_{M} \tilde{G}(x, y) P_{\tilde{g}}^{4} u(y) d V_{\tilde{g}}(y) \quad \forall x \in N ;
$$

b)

$$
\tilde{G}(x, y)=H_{0}(x, y)+K_{0}(x, y) \quad \forall x \neq y,
$$

is smooth on $N^{2} \backslash \operatorname{Diag}\left(N^{2}\right), K$ extends to a $C^{2+\alpha}$ function on $N^{2}$ and

$$
H(x, y)=\frac{1}{8 \pi^{2}} f(r) \log \frac{1}{r},
$$

where $r=d_{\tilde{g}}(x, y)$ is the geodesic distance from $x$ to $y, f(r)$ is a $C^{\infty}$ positive decreasing function, $f(r)=1$ in a neighborhood of $r=0$ and $f(r)=0$ for $r \geq \operatorname{inj}_{\tilde{g}}(N)$.

Now we are ready to make the proof of Proposition 2.4.

Proof of Proposition 2.4. We have that by assumption $u \in H_{\partial n}$ is a weak solution to

$$
\begin{cases}P_{g}^{4} u+f=\bar{f} e^{4 u} & \text { in } M, \\ P_{g}^{3} u=h & \text { on } \partial M .\end{cases}
$$

Then using Lemma 2.3 we obtain that

$$
u(x)-\bar{u}=\int_{M} G(x, y)\left(\bar{f} e^{4 u}-f\right) d V_{g}(y)+2 \int_{\partial M} G\left(x, y^{\prime}\right) h\left(y^{\prime}\right) d S_{g}\left(y^{\prime}\right) .
$$

Now let us define the following auxiliary functions

$$
w(x)=\int_{M} G(x, y) \bar{f} e^{4 u(y)} d V_{g}(y), \quad x \in M,
$$

and

$$
v(x)=-\int_{M} G(x, y) f d V_{g}(y)+2 \int_{\partial M} G\left(x, y^{\prime}\right) h\left(y^{\prime}\right) d S_{g}\left(y^{\prime}\right) .
$$

Then it is trivially seen that

$$
w(x)=u(x)-v(x), \quad x \in M .
$$

On the other hand, since $f \in C^{\infty}(M)$ and $h \in C^{\infty}(\partial M)$, then one can easily check that

$$
v \in C^{\infty}(M)
$$


Now using the relation (2.6) we obtain $w$ satisfies the following integral equation

$$
w(x)=\int_{M} G(x, y) e^{-4 v(y)} \bar{f} e^{4 w(y)} d V_{g}(y), \quad x \in M,
$$

and

$$
\frac{\partial w}{\partial n_{g}}=0 \quad \text { on } \partial M
$$

Now let us define the even reflection of $w$ through $\partial M$

$$
\tilde{w}(x)= \begin{cases}w(x) & \text { if } x \in M, \\ w(\bar{x}) & \text { if } x \in N \backslash M,\end{cases}
$$

where $N$ is the closed 4-manifold given by Lemma 2.5 .

Thanks to the fact that $\frac{\partial w}{\partial n_{g}}=0$, we have that $\tilde{w} \in H^{2}(N)$. Moreover, using the integral equation solved by $w$ (see (2.8)), one can check easily that $\tilde{w}$ satisfies

$$
\tilde{w}(x)=\int_{N} \tilde{G}(x, y) e^{-4 \tilde{v}(y)} \bar{f} e^{4 \tilde{w}(y)} d V_{\tilde{g}}(y), \quad x \in N,
$$

where $\tilde{G}$ is the Green function of $P_{\tilde{g}}^{4}$ (see Lemma 2.6) and $\bar{v}$ is the even reflection of $v$ through $\partial M$, namely

$$
\tilde{v}(x)= \begin{cases}v(x) & \text { if } x \in M, \\ v(\bar{x}) & \text { if } x \in N \backslash M .\end{cases}
$$

Furthermore from (2.5) and the fact that $f$ and $h$ are smooth, we derive that $\tilde{v} \in C^{1}(N)$.

On the other hand, from the assumption $\operatorname{Ker} P_{g}^{4,3} \simeq \mathbb{R}$, it is easily seen that Ker $P_{\tilde{g}}^{4} \simeq \mathbb{R}$. Hence using Lemma 2.6, we have that $\tilde{w}$ is a weak solution to

$$
P_{\tilde{g}}^{4}=\bar{f} e^{-4 \tilde{v}} e^{4 \tilde{w}} \quad \text { on } N .
$$

Thus from a regularity result due to Uhlenbeck and Viaclovsky, see [21], we infer that

$$
\tilde{w} \in C^{\infty}(N) .
$$

Now restricting back to $M$, we obtain

$$
w \in C^{\infty}(M) .
$$


So using (2.6), (2.7) and (2.10), we have that

$$
u \in C^{\infty}(M)
$$

The last part of the proposition follows from the same argument.

Hence the proof of the proposition is complete.

Next we give a Moser-Trudinger-type inequality when $P_{g}^{4,3}$ is nonnegative and $\operatorname{Ker} P_{g}^{4,3} \simeq \mathbb{R}$.

Proposition 2.7. Assume $P_{g}^{4,3}$ is a non-negative operator with $\operatorname{Ker} P_{g}^{4,3} \simeq$ $\mathbb{R}$. Then we have that for all $\alpha<16 \pi^{2}$ there exists a constant $C=C(M, g, \alpha)$ such that

$$
\int_{M} e^{\alpha(u-\bar{u})^{2} /\left\langle P_{g}^{4,3} u, u\right\rangle_{L^{2}(M)}} d V_{g} \leq C,
$$

for all $u \in H_{\partial / \partial n}$, and hence

$$
\log \int_{M} e^{4(u-\bar{u})} \leq C+\frac{4}{\alpha}\left\langle P_{g}^{4,3} u, u\right\rangle_{L^{2}(M)} \quad \forall u \in H_{\partial / \partial n} .
$$

In order to make the proof of Proposition 2.7 we will need two technical lemmas. We start by stating the first one whose proof can be found in $[8$, Lemma 2.2 in the Appendix].

Lemma 2.8. For all $\alpha<16 \pi^{2}$, there exists a constant $C=C(M, g, \alpha)>0$ such that

$$
\int_{M} e^{\alpha(u-\bar{u})^{2} / \int_{M}\left|\Delta_{g} u\right|^{2} d V_{g}} d V_{g} \leq C, \quad \forall u \in H_{\partial / \partial n}
$$

The next lemma states that under the assumptions $\operatorname{Ker} P_{g}^{4,3} \simeq \mathbb{R}$ and $P_{g}^{4,3}$ non-negative, the map

$$
u \in H_{\partial / \partial n} \longrightarrow\|u\|_{P_{g}^{4,3}}=\left\langle P_{g}^{4,3} u, u\right\rangle_{L^{2}(M)}^{1 / 2}
$$

induces an equivalent norm to the standard norm of $H^{2}(M)$ on $\left\{u \in H_{\partial / \partial n} \bar{u}=\right.$ $0\}$. More precisely, we have the following.

Lemma 2.9. Suppose $\operatorname{Ker} P_{g}^{4,3} \simeq \mathbb{R}$ and $P_{g}^{4,3}$ non-negative then we have that $\|\cdot\|_{P_{g}^{4,3}}$ is an equivalent norm to $\|\cdot\|_{H^{2}}$ on $\left\{u \in H_{\partial / \partial n} \bar{u}=0\right\}$. 
Proof. First of all we have that $u \rightarrow\left(\int_{M}\left|\Delta_{g} u\right|^{2} d V_{g}\right)^{1 / 2}$ is an equivalent norm to the standard norm of $H^{2}(M)$ on $\left\{u \in H_{\partial / \partial n} \bar{u}=0\right\}$.

Now with this, to prove the lemma it is sufficient to show that $\|u\|_{P_{g}^{4,3}}$ and $\left(\int_{M}\left|\Delta_{g} u\right|^{2} d V_{g}\right)^{1 / 2}$ are equivalent norms on $\left\{u \in H_{\partial / \partial n} \bar{u}=0\right\}$.

To do so, we will use a compactness argument. First of all using the definition of $P_{g}^{4,3}$, one can check easily that the following holds

$$
\|u\|_{P_{g}^{4,3}} \leq C\left(\int_{M}\left|\Delta_{g} u\right|^{2} d V_{g}\right)^{1 / 2}
$$

Now let us show that

$$
\left(\int_{M}\left|\Delta_{g} u\right|^{2} d V_{g}\right)^{1 / 2} \leq C|| u \|_{P_{g}^{4,3}} \quad \forall u \in\left\{u \in H_{\partial / \partial n} \bar{u}=0\right\} .
$$

We argue by contradiction, suppose (2.12) does not hold, then there exists $u_{l} \in\left\{u \in H_{\partial / \partial n} \bar{u}=0\right\}$ such that

$$
\int_{M}\left(\left|\Delta_{g} u_{l}\right|^{2} d V_{g}\right)^{1 / 2}=1 \quad \text { and } \quad\left\|u_{l}\right\|_{P_{g}^{4,3}} \longrightarrow 0
$$

Now using the fact that $\int_{M}\left(\left|\Delta_{g} u_{l}\right|^{2} d V_{g}\right)^{1 / 2}=1$, we get that (up to a subsequence) $u_{l} \rightarrow u^{*}$. Moreover, using the fact that $\operatorname{Ker} P_{g}^{4,3} \simeq \mathbb{R}, P_{g}^{4,3}$ is a non-negative, $\left\|u_{l}\right\|_{P_{g}^{4,3}} \rightarrow 0$ and Rellich compactness theorem, we infer that

$$
u^{*}=0 \text {. }
$$

Next using again the fact that $\left\|u_{l}\right\|_{P_{g}^{4,3}} \rightarrow 0$ and the definition of $P_{g}^{4,3}$, we infer that

$$
\begin{gathered}
\int_{M}\left|\Delta_{g} u_{l}\right|^{2} d V_{g}+\frac{2}{3} R_{g}\left|\nabla_{g} u_{l}\right| d V_{g}-2 \int_{M} \operatorname{Ric}_{g}\left(\nabla_{g} u_{l}, \nabla_{g} u_{l}\right) d V_{g} \\
-2 \int_{\partial M} L_{g}\left(\nabla_{\hat{g}} u_{l}, \nabla_{\hat{g}} u_{l}\right) d S_{g}=o_{l}(1) .
\end{gathered}
$$

Furthermore, still by the using the Rellich compactness theorem, we obtain

$$
\frac{2}{3} R_{g}\left|\nabla_{g} u_{l}\right| d V_{g}-2 \int_{M} \operatorname{Ric}_{g}\left(\nabla_{g} u_{l}, \nabla_{g} u_{l}\right) d V_{g}=o_{l}(1)
$$


Now let $\epsilon>0$ and small, then by Lemma 2.3 in [7] and also the Rellich compactness theorem we have that

$$
-2 \int_{\partial M} L_{g}\left(\nabla_{\hat{g}} u_{l}, \nabla_{\hat{g}} u_{l}\right) d S_{g} \geq \epsilon \int_{M}\left|\Delta_{g} u_{l}\right| d V_{g}-o_{l}(1) .
$$

So using (2.13), (2.15), (2.16) and (2.17), we get

$$
o_{l}(1) \geq 1-\epsilon+o_{l}(1)
$$

Thus since $\epsilon$ is small, we arrive to a contradiction. So (2.12) is true. Hence (2.11) and (2.12) imply that the lemma is proved.

Now we are ready to make the proof of Proposition 2.7.

Proof of Proposition 2.7. First of all let us set

$$
\mathcal{H}=\left\{u \in H_{\partial / \partial n}, \bar{u}=0,\left\langle P_{g}^{4,3} u, u\right\rangle_{L^{2}(M)}=1\right\}
$$

and for $\alpha>0$

$$
J_{\alpha}(u)=\int_{M} e^{\alpha u^{2}} d V_{g}, \quad u \in \mathcal{H}
$$

We have that from Lemma 2.8 and Lemma 2.9 there exists $\alpha>0$ such that

$$
\sup _{u \in \mathcal{H}} J_{\alpha}(u)<+\infty
$$

Hence,

$$
\alpha_{0}=\sup \left\{\alpha>0: \sup _{u \in \mathcal{H}} J_{\alpha}(u)<+\infty\right\}
$$

is well defined and $0<\alpha_{0} \leq \infty$.

To prove the proposition, it is sufficient to show that

$$
\alpha_{0} \geq 16 \pi^{2}
$$

Suppose by contradiction that $\alpha_{0}<16 \pi^{2}$ and let us argue for a contradiction. 
We have that by definition of $\alpha_{0}$ there exists a family $u_{\epsilon}, \epsilon>0$, such that

$$
J_{\alpha_{0}+\epsilon}\left(u_{\epsilon}\right) \rightarrow+\infty \text {. }
$$

On the other hand, using a covering argument there exists a point $p \in M$ such that for all $r>0$

$$
\int_{B_{p}(r)} e^{\left(\alpha_{0}+\epsilon\right) u_{\epsilon}^{2}} d V_{g} \rightarrow+\infty \quad \text { as } \epsilon \rightarrow 0 .
$$

Moreover, from the fact that $u_{\epsilon} \in \mathcal{H}$ and Lemma 2.9, we can assume without loss of generality that $u_{\epsilon} \rightarrow u_{0}$. Now we claim that $u_{0}=0$. Suppose not, then by using the property of the inner product, we get

$$
\left\|u_{\epsilon}-u_{0}\right\|_{P_{g}^{4,3}}<\beta
$$

for some $\beta<1$ and for $\epsilon$ small. Hence using Lemma 2.8 and Lemma 2.9, we infer that

$$
J_{\alpha_{1}}\left(u_{\epsilon}-u_{0}\right) \leq C
$$

for some $\alpha_{1}>\alpha_{0}$. Next using the Cauchy inequality, it is easily seen that

$$
J_{\alpha_{2}}\left(u_{\epsilon}\right) \leq C
$$

for some $\alpha_{2}>\alpha_{0}$. Thus a contradiction to (2.18). Hence $u_{0}=0$.

Now suppose $p \in \partial M$.

Let us take a cut-off function $\eta \in C_{0}^{\infty}\left(B_{\delta}(p)\right), \eta=1$, on $B_{p}\left(\frac{\delta}{2}\right)$ where $\delta>0$ is a fixed positive and small number. Using the Leibniz rule, we obtain

$$
\int_{B_{p}(\delta / 2)_{+}} P_{g}^{4,3}\left(\eta u_{\epsilon}\right)\left(\eta u_{\epsilon}\right) d V_{g} \leq\left\|\eta u_{\epsilon}\right\|_{P_{g}^{4,3}} \leq 1+\epsilon^{\prime}
$$

for some $\epsilon^{\prime}>0$ such that $\frac{16 \pi^{2}}{1+\epsilon^{\prime}}>\alpha_{0}$. Now let us set

$$
\tilde{u}_{\epsilon}(s, t)= \begin{cases}\left(\eta u_{\epsilon}\right) \circ \exp _{p}(s, t), & t \geq 0 \\ \left(\eta u_{\epsilon}\right) \circ \exp _{p}(s,-t), & t \leq 0\end{cases}
$$

Then from (2.19) we derive that

$$
\int_{B^{0}(\delta)}\left|\Delta_{0} \tilde{u}_{\epsilon}\right|^{2} d x \leq 2+\epsilon^{\prime \prime}
$$

for some $\epsilon^{\prime \prime}$ small where $\Delta_{0}$ denotes the Euclidean Laplacian. 
Hence by Adams inequality, see [1], we get

$$
\int_{B^{0}(\delta)} e^{\alpha_{3} \tilde{u}_{\epsilon}^{2}} d x \leq C
$$

for some $\alpha_{3}>16 \pi^{2}$. Thus we arrive to

$$
\int_{B_{p}(\delta / 2)} e^{\alpha_{3} u_{\epsilon}^{2}} d V_{g} \leq C \int_{B^{0}(\delta)} e^{\alpha_{3} \tilde{u}_{\epsilon}^{2}} d x \leq C
$$

Hence reaching a contradiction to (2.18).

Now suppose $p \in \operatorname{int}(M)$.

In this case, following the same method as above (and in a simpler way since we do not need to use $\tilde{u}_{\epsilon}$, but $u_{\epsilon}$ itself) one gets the same contradiction. Hence the proof of the proposition is complete.

\section{Proof of Theorem 1.4}

This section is concerned about the proof of Theorem 1.4. We are going to use basically the same strategy as in [18]. Hence in many steps we will be sketchy and referring to the corresponding arguments in [18]. However, we mention that, since the underlying manifold has a boundary, we have to give attention to the possible boundary blow-up points, which was not the case in [18].

Proof of Theorem 1.4. We divide the proof in five-steps as in [18].

Step 1. There exists $N \in \mathbb{N}^{*}, N$ converging points $\left(x_{i, l}\right), i=1, \ldots, N, N$ sequences $\left(\mu_{i, l}\right), i=1, \ldots, N$, of positive real numbers converging to 0 such that the following hold:
a) $\frac{d_{g}\left(x_{i, l}, x_{j, l}\right)}{\mu_{i, l}} \longrightarrow+\infty, i \neq j, i, j=1, \ldots, N$, and $\bar{Q}_{l}\left(x_{i, l}\right) \mu_{i . l}^{4} e^{4 u_{l}\left(x_{i, l}\right)}=1$.
b) There exists $C>0$ such that $\inf _{i=1, \ldots, N} d_{g}\left(x_{i, l}, x\right)^{4} e^{4 u_{l}(x)} \leq C \quad \forall x \in$ $M, \forall l \in \mathbb{N}$.
c) For every $i=1, \ldots, N$, either 
$\left.c_{1}^{i}\right)$

$$
\begin{gathered}
x_{i, l} \longrightarrow \bar{x}_{i} \in \operatorname{int}(M) \\
v_{i, l}(x)=u_{l}\left(\exp _{x_{i, l}}\left(\mu_{i, l} x\right)\right)-u_{l}\left(x_{i, l}\right)-\frac{1}{4} \log (3) \longrightarrow V_{0}(x) \\
:=\log \left(\frac{324}{162^{2}+|x|^{2}}\right) \text { in } C_{\mathrm{loc}}^{1}\left(\mathbb{R}^{4}\right),
\end{gathered}
$$

and

$$
\lim _{R \rightarrow+\infty} \lim _{l \rightarrow+\infty} \int_{B_{x_{i, l}}\left(R \mu_{i, l}\right)} \bar{Q}_{l}(y) e^{4 u_{l}(y)} d V_{g}(y)=8 \pi^{2},
$$

or

$\left.c_{2}^{i}\right)$

$$
\begin{gathered}
x_{i, l} \longrightarrow \bar{x}_{i} \in \partial M \\
v_{i, l}(x)=u_{l}\left(\exp _{x_{i, l}}\left(\mu_{i, l} x\right)\right)-u_{l}\left(x_{i, l}\right)-\frac{1}{4} \log (3) \longrightarrow V_{0}(x) \\
:=\log \left(\frac{324}{162^{2}+|x|^{2}}\right) \text { in } C_{\mathrm{loc}}^{1}\left(\mathbb{R}_{+}^{4}\right)
\end{gathered}
$$

and

$$
\lim _{R \rightarrow+\infty} \lim _{l \rightarrow+\infty} \int_{B_{x_{i, l}}^{+}\left(R \mu_{i, l}\right)} \bar{Q}_{l}(y) e^{4 u_{l}(y)} d V_{g}(y)=4 \pi^{2} .
$$

Proof of Step 1. First of all let $x_{l} \in M$ be such that $u_{l}\left(x_{l}\right)=\max _{x \in M} u_{l}(x)$, then using the fact that $u_{l}$ blows up we infer $u_{l}\left(x_{l}\right) \longrightarrow+\infty$.

Now let $\mu_{l}>0$ be such that $\bar{Q}_{l}\left(x_{l}\right) \mu_{l}^{4} e^{4 u_{l}\left(x_{l}\right)}=1$. Since $\bar{Q}_{l} \rightarrow \bar{Q}_{0} C^{1}(M)$, $\bar{Q}_{0}>0$ and $u_{l}\left(x_{l}\right) \rightarrow+\infty$, we have that $\mu_{l} \rightarrow 0$.

Now suppose $x_{l} \rightarrow \bar{x} \in \operatorname{int}(M)$ and let $B^{0}\left(\delta \mu_{l}^{-1}\right)$ be the Euclidean ball of center 0 and radius $\delta \mu_{l}^{-1}$, with $\delta>0$ small fixed. For $x \in B^{0}\left(\delta \mu_{l}^{-1}\right)$, we set

$$
\begin{gathered}
v_{l}(x)=u_{l}\left(\exp _{x_{l}}\left(\mu_{l} x\right)\right)-u_{l}\left(x_{l}\right)-\frac{1}{4} \log (3), \\
\tilde{Q}_{l}(x)=Q_{l}\left(\exp _{x_{l}}\left(\mu_{l} x\right)\right), \\
\tilde{Q}_{l}(x)=\bar{Q}_{l}\left(\exp _{x_{l}}\left(\mu_{l} x\right)\right) \\
g_{l}(x)=\left(\exp _{x_{l}}^{*} g\right)\left(\mu_{l} x\right)
\end{gathered}
$$


Now from the Green representation formula, we have

$$
\begin{aligned}
& u_{l}(x)-\bar{u}_{l} \\
& =\int_{M} G(x, y) P_{g}^{4} u_{l}(y) d V_{g}(y)+2 \int_{\partial M} G\left(x, y^{\prime}\right) P_{g}^{3} u_{l}\left(y^{\prime}\right) d S_{g}\left(y^{\prime}\right), \quad \forall x \in M,
\end{aligned}
$$

where $G$ is the Green function of $\left(P_{g}^{4}, P_{g}^{3}\right)$ (see Lemma 2.3).

Now using Equation (1.7) and differentiating (3.5) with respect to $x$, we obtain that for $k=1,2$

$$
\left|\nabla^{k} u_{l}\right|_{g}(x) \leq \int_{M}\left|\nabla^{k} G(x, y)\right|_{g} \bar{Q}_{l}(y) e^{4 u_{l}(y)} d V_{g}+O(1)
$$

since $Q_{l} \rightarrow Q_{0}$ in $C^{1}(M)$ and $T_{l} \rightarrow T_{0}$.

Now let $y_{l} \in B_{x_{l}}\left(R \mu_{l}\right), R>0$ fixed, by using the same argument as in [18, formula 43, page 11], we obtain

$$
\int_{M}\left|\nabla^{k} G\left(y_{l}, y\right)\right|_{g} e^{4 u_{l}(y)} d V_{g}(y)=O\left(\mu_{l}^{-k}\right) .
$$

Hence we get

$$
\left|\nabla^{k} v_{l}\right|_{g}(x) \leq C
$$

Furthermore from the definition of $v_{l}$ (see (3.1)), we get

$$
v_{l}(x) \leq v_{l}(0)=-\frac{1}{4} \log (3) \quad \forall x \in \mathbb{R}^{4}
$$

Thus we infer that $\left(v_{l}\right)_{l}$ is uniformly bounded in $C^{2}(K)$ for all compact subsets $K$ of $\mathbb{R}^{4}$. Hence by the Arzelà-Ascoli theorem, we derive that

$$
v_{l} \longrightarrow V_{0} \quad \text { in } \quad C_{\mathrm{loc}}^{1}\left(\mathbb{R}^{4}\right),
$$

On the other hand, (3.8) and(3.9) imply that

$$
V_{0}(x) \leq V_{0}(0)=-\frac{1}{4} \log (3) \forall x \in \mathbb{R}^{4}
$$

Moreover from (3.7) and (3.9) we have that $V_{0}$ is Lipschitz. 
On the other hand, using the Green's representation formula for $\left(P_{g}^{4}, P_{g}^{3}\right)$, we obtain that for $x \in \mathbb{R}^{4}$ fixed and for $R$ big enough such that $x \in B^{0}(R)$

$$
\begin{aligned}
u_{l}\left(\exp _{x_{l}}\left(\mu_{l} x\right)\right)-\bar{u}_{l}= & \int_{M} G\left(\exp _{x_{l}}\left(\mu_{l} x\right), y\right) P_{g}^{4} u_{l}(y) d V_{g}(y) \\
& +2 \int_{\partial M} G\left(\exp _{x_{l}}\left(\mu_{l} x\right), y^{\prime}\right) P_{g}^{3} u_{l}\left(y^{\prime}\right) d S_{g}\left(y^{\prime}\right) .
\end{aligned}
$$

Now let us set

$$
\begin{gathered}
I_{l}(x)=2 \int_{B_{x_{l}}\left(R \mu_{l}\right)}\left(G\left(\exp _{x_{l}}\left(\mu_{l} x\right), y\right)-G\left(\exp _{x_{l}}(0), y\right)\right) \bar{Q}_{l}(y) e^{4 u_{l}(y)} d V_{g}(y), \\
\mathrm{II}_{l}(x)=2 \int_{M \backslash B_{x_{l}}\left(R \mu_{l}\right)}\left(G\left(\exp _{x_{l}}\left(\mu_{l} x\right), y\right)-G\left(\exp _{x_{l}}(0), y\right)\right) \bar{Q}_{l}(y) e^{4 u_{l}(y)} d V_{g}(y), \\
\operatorname{III}_{l}(x)=2 \int_{M}\left(G\left(\exp _{x_{l}}\left(\mu_{l} x\right), y\right)-G\left(\exp _{x_{l}}(0), y\right)\right) Q_{l}(y) d V_{g}(y)
\end{gathered}
$$

and

$$
\operatorname{IIII}_{l}(x)=\int_{\partial M}\left(G\left(\exp _{x_{l}}\left(\mu_{l} x\right), y^{\prime}\right)-G\left(\exp _{x_{l}}(0), y^{\prime}\right)\right) T_{l}\left(y^{\prime}\right) d S_{g}\left(y^{\prime}\right) .
$$

Using again the same argument as in [18] (see formula (3.15) to formula $(3.21))$, we get

$$
v_{l}(x)=\mathrm{I}_{l}(x)+\mathrm{II}_{l}(x)-\mathrm{III}_{l}(x)-\operatorname{IIII}_{l}(x)-\frac{1}{4} \log (3) .
$$

Moreover, following the same methods as in [18](see formula (3.23) to formula (3.32)), we obtain

$$
\begin{aligned}
& \lim _{l} \mathrm{I}_{l}(x)=\int_{B^{0}(R)} \frac{3}{4 \pi^{2}} \log \left(\frac{|z|}{|x-z|}\right) e^{4 V_{0}(z)} d z, \\
& \limsup _{l} \operatorname{II}_{l}(x)=o_{R}(1) \text {, } \\
& \mathrm{III}_{l}(x)=o_{l}(1)
\end{aligned}
$$

and

$$
\operatorname{IIII}_{l}(x)=o_{l}(1)
$$

Hence from $(3.9),(3.12)$ to $(3.16)$ by letting $l$ tends to infinity and after $R$ tends to infinity, we obtain $V_{0}$ satisfies the following conformally invariant 
integral equation

$$
V_{0}(x)=\int_{\mathbb{R}^{4}} \frac{3}{4 \pi^{2}} \log \left(\frac{|z|}{|x-z|}\right) e^{4 V_{0}(z)} d z-\frac{1}{4} \log (3) .
$$

Now since $V_{0}$ is Lipschitz then the theory of singular integral operator gives that $V_{0} \in C^{1}\left(\mathbb{R}^{4}\right)$.

On the other hand, by using the change of variable $y=\exp _{x_{l}}\left(\mu_{l} x\right)$, one can check that the following holds:

$$
\lim _{l \rightarrow+\infty} \int_{B_{x_{l}}\left(R \mu_{l}\right)} \bar{Q}_{l} e^{4 u_{l}} d V_{g}=3 \int_{B_{0}(R)} e^{4 V_{0}} d x .
$$

Hence (1.10) implies that $e^{V_{0}} \in L^{4}\left(\mathbb{R}^{4}\right)$.

Furthermore by a classification result by $\mathrm{Xu}$, see [22, Theorem 1.2] for the solutions of (3.17), we derive that

$$
V_{0}(x)=\log \left(\frac{2 \lambda}{\lambda^{2}+\left|x-x_{0}\right|^{2}}\right)
$$

for some $\lambda>0$ and $x_{0} \in \mathbb{R}^{4}$.

Moreover from $V_{0}(x) \leq V_{0}(0)=-\frac{1}{4} \log (3) \forall x \in \mathbb{R}^{4}$, we have that $\lambda=162$ and $x_{0}=0$, namely

$$
V_{0}(x)=\log \left(\frac{324}{162^{2}+|x|^{2}}\right) .
$$

On the other hand by letting $R$ tend to infinity in (3.18), we obtain

$$
\lim _{R \rightarrow+\infty} \lim _{l \rightarrow+\infty} \int_{B_{x_{l}}\left(R \mu_{l}\right)} \bar{Q}_{l}(y) e^{4 u_{l}(y)} d V_{g}(y)=3 \int_{\mathbb{R}^{4}} e^{4 V_{0}} d x .
$$

Moreover from a generalized Pohozaev-type identity by Xu [22] (see Theorem 1.1), we get

$$
\frac{3}{4 \pi^{2}} \int_{\mathbb{R}^{4}} e^{4 V_{0}(y)} d y=2,
$$

hence using (3.20) we derive that

$$
\lim _{R \rightarrow+\infty} \lim _{l \rightarrow+\infty} \int_{B_{x_{l}}\left(R \mu_{l}\right)} \bar{Q}_{l}(y) e^{4 u_{l}(y)} d V_{g}(y)=8 \pi^{2} .
$$

Next suppose $x_{l} \rightarrow \bar{x} \in \partial M$ and let $B_{+}^{0}\left(\delta \mu_{l}^{-1}\right)$ be the upper half-euclidean ball of center 0 and radius $\delta \mu_{l}^{-1}$, with $\delta>0$ small fixed. For $x \in B_{+}^{0}\left(\delta \mu_{l}^{-1}\right)$, we consider $v_{l}(x), \tilde{Q}_{l}(x), \tilde{Q}_{l}(x)$ and $g_{l}(x)$ as in (3.1) to (3.4). 
Repeating the same argument as above, we get $v_{l}$ is uniformly bounded in $C^{2}(K)$ for every compact set $K$ of $\mathbb{R}_{+}^{4}$. Moreover, we obtain

$$
\begin{gathered}
v_{l} \longrightarrow V_{0} \quad \text { in } \quad C_{\mathrm{loc}}^{1}\left(\mathbb{R}_{+}^{4}\right), \\
V_{0}(x) \leq V_{0}(0)=-\frac{1}{3} \log (3) \quad \forall x \in \mathbb{R}_{+}^{4},
\end{gathered}
$$

and $V_{0}$ is Lipschitz.

Now let us define

$$
\begin{gathered}
\mathrm{I}_{l}(x)=2 \int_{B_{x_{l}}^{+}\left(R \mu_{l}\right)}\left(G\left(\exp _{x_{l}}\left(\mu_{l} x\right), y\right)-G\left(\exp _{x_{l}}(0), y\right)\right) \bar{Q}_{l}(y) e^{4 u_{l}(y)} d V_{g}(y) \\
\mathrm{II}_{l}(x)=2 \int_{M \backslash B_{x_{l}}^{+}\left(R \mu_{l}\right)}\left(G\left(\exp _{x_{l}}\left(\mu_{l} x\right), y\right)-G\left(\exp _{x_{l}}(0), y\right)\right) \bar{Q}_{l}(y) e^{4 u_{l}(y)} d V_{g}(y) \\
\operatorname{III}_{l}(x)=2 \int_{M}\left(G\left(\exp _{x_{l}}\left(\mu_{l} x\right), y\right)-G\left(\exp _{x_{l}}(0), y\right)\right) Q_{l}(y) d V_{g}(y)
\end{gathered}
$$

and

$$
\operatorname{IIII}_{l}(x)=\int_{\partial M}\left(G\left(\exp _{x_{l}}\left(\mu_{l} x\right), y^{\prime}\right)-G\left(\exp _{x_{l}}(0), y^{\prime}\right)\right) T_{l}\left(y^{\prime}\right) d S_{g}\left(y^{\prime}\right)
$$

By still the same argument as above, we obtain

$$
v_{l}(x)=\mathrm{I}_{l}(x)+\mathrm{II}_{l}(x)-\mathrm{III}_{l}(x)-\operatorname{IIII}_{l}(x)-\frac{1}{4} \log (3) .
$$

Moreover, we have that

$$
\begin{gathered}
\lim _{l} \mathrm{I}_{l}(x)=\int_{B_{+}^{0}(R)} \frac{3}{4 \pi^{2}}\left(\log \frac{|z|}{|x-z|}+\log \frac{|z|}{|x-\bar{z}|}\right) e^{4 V_{0}(z)} d z \\
\underset{l}{\lim \sup _{l} \mathrm{II}_{l}(x)=o_{R}(1)} \\
\operatorname{III}_{l}(x)=o_{l}(1)
\end{gathered}
$$

and

$$
\operatorname{IIII}_{l}(x)=o_{l}(1)
$$

Hence letting $l$ tends to infinity and after $R$ tending to infinity, we derive that $V_{0}$ satisfies the following integral equation

$$
V_{0}(x)=\int_{\mathbb{R}_{+}^{4}} \frac{3}{4 \pi^{2}}\left(\log \frac{|z|}{|x-z|}+\log \frac{|z|}{|x-\bar{z}|}\right) e^{4 V_{0}(z)} d z-\frac{1}{4} \log (3) .
$$


On the other hand, from (3.22), it is easily seen that

$$
\frac{\partial V_{0}}{\partial t}=0 \quad \text { on } \quad \partial \mathbb{R}_{+}^{4}
$$

Now using the Alexandrov reflection principle and denoting $\tilde{V}_{0}$ the even reflection of $V_{0}$ through the plane $\partial \mathbb{R}_{+}^{4}$, we obtain $\tilde{V}_{0}$ solves the following conformally invariant integral equation

$$
\tilde{V}_{0}(x)=\int_{\mathbb{R}^{4}} \frac{3}{4 \pi^{2}} \log \left(\frac{|z|}{|x-z|}\right) e^{4 \tilde{V}_{0}(z)} d z-\frac{1}{4} \log (3) .
$$

On the other hand, since $V_{0}$ was Lipschitz, then $\tilde{V}_{0}$ is also. Thus using the theory of singular integral operator, we infer that $\tilde{V}_{0}$ is of class $C^{1}$. Moreover, using again the change of variable $y=\exp _{x_{l}}\left(\mu_{l} x\right)$, we get

$$
\lim _{R \rightarrow+\infty} \lim _{l \rightarrow+\infty} \int_{B_{x_{l}}^{+}\left(R \mu_{l}\right)} \bar{Q}_{l}(y) e^{4 u_{l}(y)} d V_{g}(y)=3 \int_{\mathbb{R}_{+}^{4}} e^{4 V_{0}(x)} d x
$$

So from (1.10) we infer that $\int_{\mathbb{R}_{+}^{4}} e^{4 V_{0}(x)} d x<+\infty$. Thus $e^{4 \tilde{V}_{0}} \in L^{1}\left(\mathbb{R}^{4}\right)$. Now arguing as above, we obtain

$$
\tilde{V}_{0}(x)=\log \left(\frac{324}{162^{2}+|x|^{2}}\right)
$$

and

$$
\frac{3}{4 \pi^{2}} \int_{\mathbb{R}^{4}} e^{4 \tilde{V}_{0}(y)} d y=2 .
$$

Hence from the fact the $\tilde{V}_{0}$ is the even reflection of $V_{0}$ through $\partial \mathbb{R}_{+}^{4},(3.24)$ and (3.25), we get

$$
\lim _{R \rightarrow+\infty} \lim _{l \rightarrow+\infty} \int_{B_{x_{l}}^{+}\left(R \mu_{l}\right)} \bar{Q}_{l}(y) e^{4 u_{l}(y)} d V_{g}(y)=4 \pi^{2} .
$$

Now for $k \geq 1$, we say that $\left(H_{k}\right)$ holds if there exists $k$ converging points $\left(x_{i, l}\right)_{l} i=1, \ldots, k, k$ sequences $\left(\mu_{i, l}\right) i=1, \ldots, k$ of positive real numbers converging to 0 such that the following holds: 
$\left(A_{k}^{1}\right)$

$$
\begin{aligned}
& \frac{d_{g}\left(x_{i, l}, x_{j, l}\right)}{\mu_{i, l}} \longrightarrow+\infty, \quad i \neq j, \quad i, j=1, \ldots, k, \quad \text { and } \\
& \quad \times \bar{Q}_{l}\left(x_{i, l}\right) \mu_{i . l}^{4} e^{4 u_{l}\left(x_{i, l}\right)}=1 .
\end{aligned}
$$

$\left(A_{k}^{2}\right)$

For every $i=1, \ldots, k$ either $\left(A_{k, 1}^{2, i}\right)$

$$
\begin{gathered}
x_{i, l} \longrightarrow \bar{x}_{i} \in \operatorname{int}(M), \\
v_{i, l}(x)=u_{l}\left(\exp _{x_{i, l}}\left(\mu_{i, l} x\right)\right)-u_{l}\left(x_{i, l}\right)-\frac{1}{4} \log (3) \longrightarrow V_{0}(x) \\
:=\log \left(\frac{324}{162^{2}+|x|^{2}}\right) \text { in } C_{\mathrm{loc}}^{1}\left(\mathbb{R}^{4}\right)
\end{gathered}
$$

and

$$
\lim _{R \rightarrow+\infty} \lim _{l \rightarrow+\infty} \int_{B_{x_{i, l}}\left(R \mu_{i, l}\right)} \bar{Q}_{l}(y) e^{4 u_{l}(y)}=8 \pi^{2}
$$

or

$\left(A_{k, 2}^{2, i}\right)$

$$
\begin{gathered}
x_{i, l} \longrightarrow \bar{x}_{i} \in \partial M ; \\
v_{i, l}(x)=u_{l}\left(\exp _{x_{i, l}}\left(\mu_{i, l} x\right)\right)-u_{l}\left(x_{i, l}\right)-\frac{1}{4} \log (3) \longrightarrow V_{0}(x) \\
:=\log \left(\frac{324}{162^{2}+|x|^{2}}\right) \text { in } C_{\operatorname{loc}}^{1}\left(\mathbb{R}_{+}^{4}\right)
\end{gathered}
$$

and

$$
\lim _{R \rightarrow+\infty} \lim _{l \rightarrow+\infty} \int_{B_{x_{i, l}}^{+}\left(R \mu_{i, l}\right)} \bar{Q}_{l}(y) e^{4 u_{l}(y)}=4 \pi^{2} .
$$

Clearly, by the above arguments $\left(H_{1}\right)$ holds. We now let $k \geq 1$ and assume that $\left(H_{k}\right)$ holds. We also assume that

$$
\sup _{M} R_{k, l}(x)^{4} e^{4 u_{l}(x)} \longrightarrow+\infty \quad \text { as } \quad l \longrightarrow+\infty,
$$

Now using the same argument as in $[15,18]$ one can see easily that $\left(H_{k+1}\right)$. Hence since $\left(A_{k}^{1}\right)$ and $\left(A_{k}^{2}\right)$ of $H_{k}$ imply that

$$
\int_{M} \bar{Q}(y) e^{4 u_{l}(y)} d V_{g}(y) \geq\left(2 k_{1}+k_{2}\right) 4 \pi^{2}+o_{l}(1),
$$


with $k_{1}, k_{2} \in \mathbb{N}$ and $2 k_{1}+k_{2}=k$. Thus we easily get, thanks to (1.10), that there exists a maximal $k, 1 \leq k \leq \frac{1}{4 \pi^{2}}\left(\int_{M} Q_{0}(y) d V_{g}(y)+\int_{\partial M} T_{0}\left(y^{\prime}\right) d S_{g}\left(y^{\prime}\right)\right)$, such that $\left(H_{k}\right)$ holds. Arriving to this maximal $k$, we get that (3.26) cannot hold. Hence setting $N=k$, the proof of Step 1 is done.

Step 2. There exists a constant $C>0$ such that

$$
R_{l}(x)\left|\nabla u_{l}\right|_{g}(x) \leq C \quad \forall x \in M \text { and } \forall l \in N
$$

where

$$
R_{l}(x)=\min _{i=1, \ldots, N} d_{g}\left(x_{i, l}, x\right)
$$

and the $x_{i, l}$ 's are as in Step 1 .

Proof of Step 2. First of all using the Green representation formula for $\left(P_{g}^{4}\right.$, $\left.P_{g}^{3}\right)$, see Lemma 2.3, we obtain

$$
u_{l}(x)-\bar{u}_{l}=\int_{M} G(x, y) P_{g}^{4} u_{l}(y) d V_{g}(y)+2 \int_{\partial M} G\left(x, y^{\prime}\right) P_{g}^{3} u_{l}\left(y^{\prime}\right) d S_{g}\left(y^{\prime}\right) .
$$

Now using the BVP (1.4), we get

$$
\begin{aligned}
u_{l}(x)-\bar{u}_{l}= & 2 \int_{M} G(x, y)\left(\bar{Q}_{l}(y) e^{4 u_{l}(y)}-Q_{l}\right) d V_{g}(y) \\
& -2 \int_{\partial M} G\left(x, y^{\prime}\right) T_{l}\left(y^{\prime}\right) u_{l}\left(y^{\prime}\right) d S_{g}\left(y^{\prime}\right) .
\end{aligned}
$$

Thus differentiating with respect to $x$ (3.28) and using the fact that $Q_{l} \rightarrow$ $Q_{0}, \bar{Q}_{l} \rightarrow \bar{Q}_{0}$ and $T_{l} \rightarrow T_{0}$ in $C^{1}$, we have that for $x_{l} \in M$

$$
\left|\nabla u_{l}\left(x_{l}\right)\right|_{g}=O\left(\int_{M} \frac{1}{d_{g}\left(x_{l}, y\right)} e^{4 u_{l}(y)} d V_{g}(y)\right)+O(1) .
$$

Hence at this stage following the same argument as in the proof of Theorem 1.3 , Step 2 in [18], we obtain

$$
\int_{M} \frac{1}{\left(d_{g}\left(x_{l}, y\right)\right)} e^{4 u_{l}(y)} d V_{g}(y)=O\left(\frac{1}{R_{l}\left(x_{l}\right)}\right)
$$

hence since $x_{l}$ is arbitrary, then the proof of Step 2 is complete.

Step 3. Set

$$
R_{i, l}=\min _{i \neq j} d_{g}\left(x_{i, l}, x_{j, l}\right)
$$

we have that 
1) There exists a constant $C>0$ such that $\forall r \in\left(0, R_{i, l}\right] \forall s \in\left(\frac{r}{4}, r\right]$ if $\bar{x}_{i} \in$ $\operatorname{int}(M)$, then

$$
\begin{aligned}
& \left|u_{l}\left(\exp _{x_{i, l}}(r x)\right)-u_{l}\left(\exp _{x_{i, l}}(s y)\right)\right| \leq C \\
& \quad \text { for all } x, y \in \mathbb{R}^{4} \text { such that }|x|,|y| \leq \frac{3}{2},
\end{aligned}
$$

and if $\bar{x}_{i} \in \partial M$, then

$$
\begin{aligned}
& \left|u_{l}\left(\exp _{x_{i, l}}(r x)\right)-u_{l}\left(\exp _{x_{i, l}}(s y)\right)\right| \leq C \\
& \quad \text { for all } x, y \in \mathbb{R}_{+}^{4} \text { such that }|x|,|y| \leq \frac{3}{2}
\end{aligned}
$$

2) If $d_{i, l}$ is such that $0<d_{i, l} \leq \frac{R_{i, l}}{2}$ and $\frac{d_{i, l}}{\mu_{i, l}} \rightarrow+\infty$, then we have that if $\bar{x}_{i} \in \operatorname{int}(M)$ and

$$
\int_{B_{x_{i, l}}\left(d_{i, l}\right)} \bar{Q}_{l}(y) e^{4 u_{l}(y)} d V_{g}(y)=8 \pi^{2}+o_{l}(1),
$$

then

$$
\int_{B_{x_{i, l}}\left(2 d_{i, l}\right)} \bar{Q}_{l}(y) e^{4 u_{l}(y)} d V_{g}(y)=8 \pi^{2}+o_{l}(1) .
$$

If $\bar{x}_{i} \in \partial M$ and

$$
\int_{B_{x_{i, l}}^{+}\left(d_{i, l}\right)} \bar{Q}_{l}(y) e^{4 u_{l}(y)} d V_{g}(y)=4 \pi^{2}+o_{l}(1),
$$

then

$$
\int_{B_{x_{i, l}}^{+}\left(2 d_{i, l}\right)} \bar{Q}_{l}(y) e^{4 u_{l}(y)} d V_{g}(y)=4 \pi^{2}+o_{l}(1) .
$$

$3)$ Let $R$ be large and fixed. If $d_{i, l}>0$ is such that $d_{i, l} \rightarrow 0, \frac{d_{i, l}}{\mu_{i, l}} \rightarrow+\infty$, $d_{i, l}<\frac{R_{i, l}}{4 R}$, then if $\bar{x}_{i} \in \operatorname{int}(M)$ and

$$
\int_{B_{x_{i, l}}\left(d_{i, l} / 2 R\right)} \bar{Q}_{l}(y) e^{4 u_{l}(y)} d V_{g}(y)=8 \pi^{2}+o_{l}(1),
$$

then by setting

$$
\tilde{u}_{l}(x)=u_{l}\left(\exp _{x_{i, l}}\left(d_{i, l} x\right)\right), \quad x \in A_{2 R},
$$


where $A_{2 R}=B^{0}(2 R) \backslash B^{0}\left(\frac{1}{2 R}\right)$, we have that

$$
\left\|d_{i, l}^{4} e^{4 \tilde{u}_{l}}\right\|_{C^{\alpha}\left(A_{R}\right)} \longrightarrow 0 \quad \text { as } l \longrightarrow+\infty
$$

for some $\alpha \in(0,1)$, where $A_{R}=B^{0}(R) \backslash B^{0}\left(\frac{1}{R}\right)$; and if $\bar{x}_{i} \in \partial M$ and

$$
\int_{B_{x_{i, l}}^{+}\left(d_{i, l} / 2 R\right)} \bar{Q}_{l}(y) e^{4 u_{l}(y)} d V_{g}(y)=4 \pi^{2}+o_{l}(1)
$$

then by setting

$$
\tilde{u}_{l}(x)=u_{l}\left(\exp _{x_{i, l}}\left(d_{i, l} x\right)\right), \quad x \in A_{2 R}^{+}
$$

where $A_{2 R}^{+}=B_{+}^{0}(2 R) \backslash B_{+}^{0}\left(\frac{1}{2 R}\right)$, we have that

$$
\left\|d_{i, l}^{4} e^{4 \tilde{u}_{l}}\right\|_{C^{\alpha}\left(A_{R}^{+}\right)} \longrightarrow 0 \text { as } l \longrightarrow+\infty
$$

for some $\alpha \in(0,1)$, where $A_{R}^{+}=B_{+}^{0}(R) \backslash B_{+}^{0}\left(\frac{1}{R}\right)$.

Proof of Step 3. We have that property 1 follows immediately from Step 2 and the definition of $R_{i, l}$. In fact, we can join $r x$ to $s y$ by a curve whose length is bounded by a constant proportional to $r$.

Now let us show point 2. First suppose $\bar{x}_{i} \in \operatorname{int}(M)$. From $\frac{d_{i, l}}{\mu_{i, l}} \rightarrow+\infty$, point c of Step 1 and (3.31), we have that

$$
\int_{B_{x_{i, l}}\left(d_{i, l}\right) \backslash B_{x_{i, l}}\left(d_{i, l} / 2\right)} e^{4 u_{l}(y)} d V_{g}(y)=o_{l}(1) .
$$

Hence from (3.29), by taking $s=\frac{r}{2}$ and $r=2 d_{i, l}$, we obtain that

$$
\int_{B_{x_{i, l}}\left(2 d_{i, l}\right) \backslash B_{x_{i, l}}\left(d_{i, l}\right)} e^{4 u_{l}(y)} d V_{g}(y) \leq C \int_{B_{x_{i, l}}\left(d_{i, l}\right) \backslash B_{x_{i, l}}\left(d_{i, l} / 2\right)} e^{4 u_{l}(y)} d V_{g}(y) .
$$

Thus we get

$$
\int_{B_{x_{i, l}}\left(2 d_{i, l}\right) \backslash B_{x_{i, l}}\left(d_{i, l}\right)} e^{4 u_{l}(y)} d V_{g}(y)=o_{l}(1) .
$$


Next assume $\bar{x}_{i} \in \partial M$. Thanks to $\frac{d_{i, l}}{\mu_{i, l}} \longrightarrow+\infty$, point c of Step 1 and (3.32), we have that

$$
\int_{B_{x_{i, l}}^{+}\left(d_{i, l}\right) \backslash B_{x_{i, l}}^{+}\left(d_{i, l} / 2\right)} e^{4 u_{l}(y)} d V_{g}(y)=o_{l}(1) .
$$

Thus using (3.30), with $s=\frac{r}{2}$ and $r=2 d_{i, l}$, we get

$$
\int_{B_{x_{i, l}}^{+}\left(2 d_{i, l}\right) \backslash B_{x_{i, l}}^{+}\left(d_{i, l}\right)} e^{4 u_{l}(y)} d V_{g}(y) \leq C \int_{B_{x_{i, l}}^{+}\left(d_{i, l}\right) \backslash B_{x_{i, l}}^{+}\left(d_{i, l} / 2\right)} e^{4 u_{l}(y)} d V_{g}(y) .
$$

Hence we arrive

$$
\int_{B_{x_{i, l}}^{+}\left(2 d_{i, l}\right) \backslash B_{x_{i, l}}^{+}\left(d_{i, l}\right)} e^{4 u_{l}(y)} d V_{g}(y)=o_{l}(1) .
$$

So the proof of point 2 is done. On the other hand, by following in a straightforward way the proof of point 3 in Step 3 of Theorem 1.3 in [18], one gets easily point 3 . Hence the proof of Step 3 is complete.

Step 4. There exists a positive constant $C$ independent of $l$ and $i$ such that if $\bar{x}_{i} \in \operatorname{int}(M)$, then

$$
\int_{B_{x_{i, l}}\left(R_{i, l} / C\right)} \bar{Q}_{l}(y) e^{4 u_{l}(y)} d V_{g}(y)=8 \pi^{2}+o_{l}(1)
$$

and if $\bar{x}_{i} \in \partial M$, then

$$
\int_{B_{x_{i, l}}^{+}\left(R_{i, l} / C\right)} \bar{Q}_{l}(y) e^{4 u_{l}(y)} d V_{g}(y)=4 \pi^{2}+o_{l}(1)
$$

Proof of Step 4. The proof is an adaptation of the arguments in Step 4 [18], but for the readers convenience we will make it.

First of all fix $\frac{1}{4}<\nu<\frac{1}{2}$, and for $i=1, \ldots, N$ if $\bar{x}_{i} \in \operatorname{int}(M)$, then set

$$
\begin{gathered}
\bar{u}_{i, l}(r)=\operatorname{Vol}_{g}\left(\partial B_{x_{i, l}}(r)\right)^{-1} \int_{\partial B_{x_{i, l}}(r)} u_{l}(x) d \sigma_{g}(x) \quad \forall 0 \leq r<\operatorname{inj}_{g}(M) \\
\varphi_{i, l}(r)=r^{4 \nu} \exp \left(\bar{u}_{i, l}(r)\right) \quad \forall 0 \leq r<\operatorname{inj}_{g}(M)
\end{gathered}
$$


if $\bar{x}_{i} \in \partial M$, then set

$$
\begin{gathered}
\bar{u}_{i, l}(r)=\operatorname{Vol}_{g}\left(\partial B_{x_{i, l}}^{+}(r)\right)^{-1} \int_{\partial B_{x_{i, l}}^{+}(r)} u_{l}(x) d \sigma_{g}(x) \quad \forall 0 \leq r<\operatorname{inj}_{g}(M) \\
\varphi_{i, l}(r)=r^{4 \nu} \exp \left(\bar{u}_{i, l}(r)\right) \quad \forall 0 \leq r<\operatorname{inj}_{g}(M) .
\end{gathered}
$$

By assumption c or d of Step 1, we have that there exists $R_{\nu}$ such that

$$
\forall R \geq R_{\nu}, \varphi_{i, l}^{\prime}\left(R \mu_{i, l}\right)<0 \quad \forall l \text { sufficiently large (depending on } R \text { ). }
$$

Now we define $r_{i, l}$ by

$$
r_{i, l}=\sup \left\{R_{\nu} \mu_{i, l} \leq r \leq \frac{R_{i, l}}{2} \text { s.t. } \varphi_{i, l}^{\prime}(\cdot)<0 \text { in }\left[R_{\nu}, r\right)\right\} .
$$

Hence (3.35) implies that

$$
\frac{r_{i, l}}{\mu_{i, l}} \longrightarrow+\infty \quad \text { as } l \longrightarrow+\infty
$$

Now to prove the step, it suffices to show that $\frac{R_{i, l}}{r_{i, l}} \nrightarrow+\infty$ as $l \longrightarrow+\infty$.

Indeed if $\frac{R_{i, l}}{r_{i, l}} \nrightarrow+\infty$, we have that there exists a positive constant $C$ such that

$$
\frac{R_{i, l}}{C} \leq r_{i, l}
$$

On the other hand, from the Harnack-type inequality (3.29) or (3.30), point c or d of Step 1 and (3.36), we have that for any $\eta>0$, there exists $R_{\eta}>0$ such that for any $R>R_{\eta}$, we have that

$$
d_{g}\left(x, x_{i, l}\right)^{4 \nu} e^{4 u_{l}} \leq \eta \mu_{i, l}^{4(\nu-1)} \quad \forall x \in\left(B_{x_{i, l}}^{+}\left(r_{i, l}\right) \backslash B_{x_{i, l}}^{+}\left(R \mu_{i, l}\right)\right) .
$$

Since $\frac{r_{i, l}}{\mu_{i, l}} \longrightarrow+\infty$, see $(3.37)$, and $\frac{R_{i, l}}{2} \geq r_{i, l}$, see (3.36), we have $\frac{R_{i, l}}{C \mu_{i, l}} \longrightarrow$ $+\infty$, hence point $\mathrm{c}$ or $\mathrm{d}$ of Step 1, (3.39) and (3.38) imply that if $\bar{x}_{i} \in \operatorname{int}(M)$, then

$$
\int_{B_{x_{i, l}}\left(R_{i, l} / C\right)} \bar{Q}_{l} e^{4 u_{l}}=8 \pi^{2}+o_{l}(1)
$$

and if $\bar{x}_{i} \in \partial M$, then

$$
\int_{B_{x_{i, l}}^{+}\left(R_{i, l} / C\right)} \bar{Q}_{l} e^{4 u_{l}}=4 \pi^{2}+o_{l}(1)
$$


On the other hand, by continuity and by the definition of $r_{i, l}$, it follows that

$$
\varphi_{i, l}^{\prime}\left(r_{i, l}\right)=0
$$

Let us assume by contradiction that $\frac{R_{i, l}}{r_{i, l}} \longrightarrow+\infty$. We will show next that $\varphi_{i, l}^{\prime}\left(r_{i, l}\right)<0$ for $l$ large, contradicting the above equality (3.40). To do so, we will study $\bar{u}_{i, l}(\cdot)$.

First let us remark that since $M$ is compact, then $\frac{R_{i, l}}{r_{i, l}} \longrightarrow+\infty$ implies that $r_{i, l} \longrightarrow 0$.

From Green's representation formula for $u_{l}$, we have the following equation:

$$
\begin{aligned}
u_{l}(x)= & \int_{M} G(x, y) P_{g}^{4} u_{l}(y) d V_{g}(y)+\bar{u}_{l}+2 \int_{M} G(x, y) P_{g}^{3} u_{l}\left(y^{\prime}\right) d S_{g}\left(y^{\prime}\right) \\
= & \int_{M} G(x, y) \bar{Q}_{l}(y) e^{4 u_{l}(y)} d V_{g}(y)+\bar{u}_{l}-\int_{M} G(x, y) Q_{l}(y) d V_{g}(y) \\
& -2 \int_{\partial M} G\left(x, y^{\prime}\right) P_{g}^{3} u_{l}\left(y^{\prime}\right) d S_{g}\left(y^{\prime}\right) .
\end{aligned}
$$

Hence

$$
\begin{aligned}
\bar{u}_{i, l}(r)= & 2\left(\operatorname{Vol}_{g}\left(\partial B_{x_{i, l}}^{+}(r)\right)\right)^{-1} \int_{\partial B_{x_{i, l}}^{+}(r)} \int_{M} G(x, y) \bar{Q}_{l}(y) e^{4 u_{l}(y)} d V_{g}(y) d \sigma_{g}(x) \\
& +\bar{u}_{l}-2\left(\operatorname{Vol}_{g}\left(\partial B_{x_{i, l}^{+}}^{+}(r)\right)\right)^{-1} \int_{\partial B_{x_{i, l}}^{+}(r)} \int_{M} G(x, y) Q_{l}(y) d V_{g}(y) d \sigma_{g}(x) \\
& -\left(\operatorname{Vol}_{g}\left(\partial B_{x_{i, l}}^{+}(r)\right)\right)^{-1} \int_{\partial B_{x_{i, l}}^{+}(r)} \int_{\partial M} G(x, y) T_{l}(y) d S_{g}(y) d \sigma_{g}(x)
\end{aligned}
$$

Setting

$$
\begin{aligned}
F_{i, l}(r)= & 2\left(\operatorname{Vol}_{g}\left(\partial B_{x_{i, l}}^{+}(r)\right)\right)^{-1} \int_{\partial B_{x_{i, l}}^{+}(r)} \int_{M} G(x, y) Q_{l}(y) d V_{g}(y) d \sigma_{g}(x) \\
& +\left(\operatorname{Vol}_{g}\left(\partial B_{x_{i, l}}^{+}(r)\right)^{-1} \int_{\partial B_{x_{i, l}}^{+}(r)} \int_{\partial M} G(x, y) T_{l}(y) d S_{g}(y) d \sigma_{g}(x),\right.
\end{aligned}
$$

we obtain

$$
\begin{aligned}
\bar{u}_{i, l}= & 2\left(\operatorname{Vol}_{g}\left(\partial B_{x_{i, l}}^{+}(r)\right)\right)^{-1} \int_{\partial B_{x_{i, l}}^{+}(r)} \int_{M} G(x, y) \bar{Q}_{l}(y) e^{4 u_{l}(y)} d V_{g}(y) d \sigma_{g}(x) \\
& +\bar{u}_{l}-F_{i, l}(r) .
\end{aligned}
$$


Since $Q_{l} \longrightarrow Q_{0}$ in $C^{1}(M)$ and $T_{l} \longrightarrow T_{0}$ in $C^{1}(\partial M)$, then we have that $F_{i, l}$ is of class $C^{1}$ for all $i, l$, and moreover,

$$
\left|F_{i, l}^{\prime}(r)\right| \leq C, \quad \forall r \in\left(0, \frac{\operatorname{inj}_{g}(M)}{4}\right)
$$

Now let $\frac{\operatorname{inj}_{g}(M)}{4}<A<\frac{\operatorname{inj}_{g}(M)}{2}$ be fixed: we have that

$$
\begin{aligned}
\int_{M} G(x, y) \bar{Q}_{l}(y) e^{4 u_{l}(y)} d V_{g}(y)= & \int_{B_{x_{i, l}}^{+}(A)} G(x, y) \bar{Q}_{l} e^{4 u_{l}(y)} d V_{g}(y) \\
& +\int_{M \backslash B_{x_{i, l}}(A)} G(x, y) \bar{Q}_{l} e^{4 u_{l}(y)} d V_{g}(y) .
\end{aligned}
$$

So

$$
\begin{aligned}
\bar{u}_{i, l}(r)= & \left.2 \operatorname{Vol}_{g}\left(\partial B_{x_{i, l}}^{+}(r)\right)\right)^{-1} \int_{\partial B_{x_{i, l}}^{+}(r)} \int_{B_{x_{i, l}}^{+}(A)}(G(x, y)-K(x, y)) \\
& \times \bar{Q}_{l}(y) e^{4 u_{l}(y)} d V_{g}(y) d \sigma_{g}(x)+\bar{u}_{l}-F_{i, l}(r)+H_{i, l}(r)
\end{aligned}
$$

with

$$
\begin{aligned}
H_{i, l}(r)= & 2 \operatorname{Vol}_{g}\left(\partial B_{x_{i, l}}^{+}(r)\right)^{-1} \int_{\partial B_{x_{i, l}}^{+}(r)} \int_{M \backslash B_{x_{i, l}}(A)} G(x, y) \\
& \left.\times \bar{Q}_{l}(y) e^{4 u_{l}(y)} d V_{g}(y) d \sigma_{g}(x)+2 \operatorname{Vol}_{g}\left(\partial B_{x_{i, l}}^{+}(r) \cap M\right)\right)^{-1} \\
& \times \int_{\partial B_{x_{i, l}}^{+}(r)} \int_{B_{x_{i, l}}^{+}(A)} K(x, y) \bar{Q}_{l}(y) e^{4 u_{l}(y)} d V_{g}(y) d \sigma_{g}(x) .
\end{aligned}
$$

Since $G$ is smooth out of $\operatorname{Diag}(M)$, then for all $i, l, H_{i, l} \in C^{1}\left(0, \frac{\operatorname{inj}_{g}(M)}{4}\right)$, and moreover,

$$
\left|H_{i, l}^{\prime}(r)\right| \leq C \quad \forall r \in\left(0, \frac{\operatorname{inj}_{g}(M)}{4}\right)
$$

To continue the proof of the step we divide it into two cases:

Case 1. $\bar{x}_{i} \in \operatorname{int}(M)$ 
First of all using the change of variable $x=r \theta$ and $y=s \tilde{\theta}$, we obtain

$$
\begin{aligned}
\bar{u}_{i, l}= & \left(\operatorname{Vol}\left(S^{3}\right)\right)^{-1} \int_{S^{3}} \int_{S^{3}} \int_{0}^{A} f(r, \theta)(G(r \theta, s \tilde{\theta})-K(r \theta, s \tilde{\theta})) \\
& \times \bar{Q}(s \tilde{\theta}) e^{4 u_{l}(s \tilde{\theta})} s^{3} f(s, \tilde{\theta}) d s d \tilde{\theta} d \theta+\bar{u}_{l}-F_{i, l}(r)+H_{i, l}(r) .
\end{aligned}
$$

So differentiating with respect to $r$, we have that

$$
\begin{aligned}
\bar{u}_{i, l}^{\prime}(r)= & \left(\operatorname{Vol}\left(S^{3}\right)\right)^{-1} \int_{S^{3}} \int_{S^{3}} \int_{0}^{A} \frac{\partial}{\partial r}(f(r, \theta)(G(r \theta, s \tilde{\theta})-K(r \theta, s \tilde{\theta}))) \\
& \times \bar{Q}(s \tilde{\theta}) e^{4 u_{l}(s \tilde{\theta})} s^{3} f(s, \tilde{\theta}) d s d \tilde{\theta} d \theta-F_{i, l}^{\prime}(r)+H_{i, l}^{\prime}(r) .
\end{aligned}
$$

From the asymptotics of $G(\cdot, \cdot)$ (see Lemma 2.3) and the fact that $f$ is bounded in $C^{2}$, it follows that

$$
\begin{aligned}
& \left(\operatorname{Vol}\left(S^{3}\right)\right)^{-1} \int_{S^{3}} \int_{S^{3}}(G(r \theta, s \tilde{\theta})-K(r \theta, s \tilde{\theta})) d \tilde{\theta} d \theta \\
& =\hat{f}(r, s) \log \left(\frac{1}{|r-s|}\right)+H(r, s),
\end{aligned}
$$

with $H(\cdot, \cdot)$ of class $C^{\alpha}$ and $\hat{f}(\cdot, \cdot)$ of class $C^{2}$.

Hence setting

$$
\begin{aligned}
\tilde{G}(r, s)= & \left(\operatorname{Vol}\left(S^{3}\right)\right)^{-1} \int_{S^{3}} \int_{S^{3}} \frac{\partial}{\partial r}(f(r, \theta)(G(r \theta, s \tilde{\theta})-K(r \theta, s \tilde{\theta}))) \\
& \times \bar{Q}(s \tilde{\theta}) f(s, \tilde{\theta}) d \tilde{\theta} d \theta
\end{aligned}
$$

we obtain

$$
\tilde{G}(r, s)=\hat{f}(r, s) \frac{1}{r-s}+\tilde{H}(r, s)
$$

where $\tilde{H}(r, \cdot)$ is integrable for every $r$ fixed.

On the other hand, using the Harnack-type inequality (see (3.29)), we have that

$$
u_{l}(s \tilde{\theta}) \leq \bar{u}_{i, l}(s)+C \text { uniformly in } \tilde{\theta}
$$

hence we obtain

$$
\bar{u}_{i, l}(r) \leq C \int_{0}^{A} s^{3} \tilde{G}(r, s) e^{n \bar{u}_{i, l}(s)} d s-F_{i, l}^{\prime}(r)+H_{i, l}^{\prime}(r) .
$$


Now we study $\int_{0}^{A} s^{3} \tilde{G}(r, s) e^{4 \bar{u}_{i, l}(s)} d s$. To do so, let $R$ be so large such that $r_{i, l} \leq \frac{R_{i, l}}{4 R}$ (this is possible because of the assumption of contradiction). Now let us split the integral in the following way:

$$
\begin{aligned}
\int_{0}^{A} & s^{3} \tilde{G}(r, s) e^{n \bar{u}_{i, l}(s)} d s \\
= & \int_{0}^{r_{i, l} / R} s^{3} \tilde{G}(r, s) e^{4 \bar{u}_{i, l}(s)} d s+\int_{r_{i, l} / R}^{r_{i, l} R} s^{3} \tilde{G}(r, s) e^{4 \bar{u}_{i, l}(s)} d s \\
& \quad+\int_{r_{i, l} R}^{R_{i, l} / C} s^{3} \tilde{G}(r, s) e^{4 \bar{u}_{i, l}(s)} d s+\int_{R_{i, l} / C}^{A} s^{3} \tilde{G}(r, s) e^{n \bar{u}_{i, l}(s)} d s .
\end{aligned}
$$

Using the fact that we are at the scale $\frac{r_{i, l}}{R}$, then c of Step 1 implies that we have the following estimates for the first term of the equality above with $r=r_{i, l}$,

$$
\int_{0}^{r_{i, l} / R} s^{3} \tilde{G}\left(r_{i, l}, s\right) e^{4 \bar{u}_{i, l}(s)} d s=-\frac{2}{r_{i, l}}+o_{l}(1) \frac{1}{r_{i, l}} .
$$

On the other hand, using assumption b of Step 1, we obtain the following estimate for the third term of the equality above with $r=r_{i, l}$

$$
\int_{r_{i, l} R}^{R_{i, l} / C} s^{3} \tilde{G}\left(r_{i, l}, s\right) e^{4 \bar{u}_{i, l}(s)} d s=o_{l}(1) \frac{1}{r_{i, l}} .
$$

We have also using assumption b of Step 1 and the fact that $\frac{R_{i, l}}{r_{i, l}} \longrightarrow+\infty$ the following estimate for the fourth still with $r=r_{i, l}$,

$$
\int_{R_{i, l} / C}^{A} s^{3} \tilde{G}\left(r_{i, l}, s\right) e^{4 \bar{u}_{i, l}(s)} d s=o_{l}(1) \frac{1}{r_{i, l}} .
$$

Now let us estimate the second term. For this, we will use point 3 of Step 3. First we recall that $r_{i, l}$ and $R$ verify the assumption of the latter. Hence the following holds:

$$
\left\|r_{i, l}^{4} e^{4 \tilde{u}_{l}}\right\|_{C^{\alpha}\left(A_{R}\right)}=o_{l}(1)
$$

for the definition of $A_{R}$ and $\tilde{u}_{l}$, see the statement of point 3 of Step 3 where $d_{i, l}$ is replaced by $r_{i, l}$. On the other hand, performing a change of variable 
say $r_{i, l} y=s$ we obtain the following equality:

$$
\int_{r_{i, l} / R}^{r_{i, l} R} s^{3} \tilde{G}(r, s) e^{4 \bar{u}_{i, l}(s)} d s=\int_{1 / R}^{R} y^{3} \hat{G}_{i, l}(y) r_{i, l}^{4} e^{4 \hat{u}_{i, l}(y)} d y
$$

where

$$
\begin{aligned}
\hat{u}_{i, l}(y) & =\bar{u}_{i, l}\left(r_{i, l} y\right), \\
\hat{G}_{i, l}(y) & =\tilde{G}\left(r_{i, l}, r_{i, l} y\right) .
\end{aligned}
$$

From the asymptotics of $\tilde{G}(\cdot, \cdot)$ (see $(3.43))$ we deduce the following one for $\hat{G}_{i, l}(\cdot, \cdot)$,

$$
\hat{G}_{i, l}(y)=\hat{f}_{i, l}(y) \frac{1}{r_{i, l}(1-y)}+\hat{H}_{i, l}(y)
$$

where $\hat{H}_{i, l}(\cdot)$ is integrable and $\hat{f}_{i, l}(\cdot)$ of class $C^{2}$.

Hence by using (3.45) and (3.53), we obtain the following inequality:

$$
\begin{aligned}
& \int_{r_{i, l} / R}^{r_{i, l} R} s^{3} \tilde{G}\left(r_{i, l}, s\right) e^{4 \bar{u}_{i, l}(s)} d s \\
& \quad=\frac{1}{r_{i, l}} \int_{1 / R}^{R} y^{3}\left(\frac{\hat{f}_{i, l}(y)}{(1-y)}+r_{i, l} \hat{H}_{i, l}(y)\right) r_{i, l}^{4} e^{4 \hat{u}_{i, l}(y)} d y .
\end{aligned}
$$

Moreover, using the Harnack-type inequality for $u_{l}$ (see (3.29) and (3.44)), we have that holds

$$
\left\|r_{i, l}^{4} e^{4 \hat{u}_{i, l}}\right\|_{C^{\alpha}(](1 / R), R[)}=o_{l}(1)
$$

So using techniques of the theory of singular integral operators as in Lemma 4.4 [17] to have Holder estimates, we obtain

$$
\int_{1 / R}^{R} y^{3}\left(\frac{\hat{f}_{i, l}(y)}{(1-y)}+r_{i, l} \hat{H}_{i, l}(y)\right) r_{i, l}^{4} e^{4 \hat{u}_{i, l}(y)} d y=o_{l}(1)
$$

hence with (3.45) we deduce that

$$
\int_{r_{i, l} / R}^{r_{i, l} R} s^{3} \tilde{G}(r, s) e^{4 \bar{u}_{i, l}(s)} d s=o_{l}\left(\frac{1}{r_{i, l}}\right) .
$$


So we obtain

$$
\bar{u}_{i, l}^{\prime}\left(r_{i, l}\right) \leq-2 C \frac{1}{r_{i, l}}+o_{l}(1) \frac{1}{r_{i, l}}-F_{i, l}^{\prime}\left(r_{i, l}\right)+H_{i, l}^{\prime}(r) .
$$

Case 2. $\bar{x}_{i} \in \partial M$

We will follow the same strategy up to some trivial adaptations. First using the change of variable $x=r \theta$ and $y=s \tilde{\theta}$, we obtain

$$
\begin{aligned}
\bar{u}_{i, l}= & \left(\operatorname{Vol}\left(S_{+}^{3}\right)\right)^{-1} \int_{S_{+}^{3}} \int_{S_{+}^{3}} \int_{0}^{A} f(r, \theta)(G(r \theta, s \tilde{\theta})-K(r \theta, s \tilde{\theta})) \bar{Q}(s \tilde{\theta}) e^{4 u_{l}(s \tilde{\theta})} \\
& \times s^{3} f(s, \tilde{\theta}) d s d \tilde{\theta} d \theta+\bar{u}_{l}-F_{i, l}(r)+H_{i, l}(r) .
\end{aligned}
$$

So differentiating with respect to $r$, we have that

$$
\begin{aligned}
\bar{u}_{i, l}^{\prime}(r)= & \left(\operatorname{Vol}\left(S_{+}^{3}\right)\right)^{-1} \int_{S_{+}^{3}} \int_{S_{+}^{3}} \int_{0}^{A} \frac{\partial}{\partial r}(f(r, \theta)(G(r \theta, s \tilde{\theta})-K(r \theta, s \tilde{\theta}))) \\
& \times \bar{Q}(s \tilde{\theta}) e^{4 u_{l}(s \tilde{\theta})} s^{3} f(s, \tilde{\theta}) d s d \tilde{\theta} d \theta-F_{i, l}^{\prime}(r)+H_{i, l}^{\prime}(r) .
\end{aligned}
$$

From the asymptotics of $G(\cdot, \cdot)$ (see Lemma 2.3) and the fact that $f$ is bounded in $C^{2}$, it follows that

$$
\begin{aligned}
& \left(\operatorname{Vol}\left(S^{3}\right)\right)^{-1} \int_{S_{+}^{3}} \int_{S_{+}^{3}}(G(r \theta, s \tilde{\theta})-K(r \theta, s \tilde{\theta})) d \tilde{\theta} d \theta \\
& \quad=\hat{f}(r, s) \log \left(\frac{1}{|r-s|}\right)+H(r, s)
\end{aligned}
$$

with $H(\cdot, \cdot)$ of class $C^{\alpha}$ and $\hat{f}(\cdot, \cdot)$ of class $C^{2}$.

Hence setting

$$
\begin{aligned}
\tilde{G}(r, s)= & \left(\operatorname{Vol}\left(S_{+}^{3}\right)\right)^{-1} \int_{S_{+}^{3}} \int_{S_{+}^{3}} \frac{\partial}{\partial r}(f(r, \theta)(G(r \theta, s \tilde{\theta})-K(r \theta, s \tilde{\theta}))) \\
& \times \bar{Q}(s \tilde{\theta}) f(s, \tilde{\theta}) d \tilde{\theta} d \theta
\end{aligned}
$$

we obtain

$$
\tilde{G}(r, s)=\hat{f}(r, s) \frac{1}{r-s}+\tilde{H}(r, s),
$$

where $\tilde{H}(r, \cdot)$ is integrable for every $r$ fixed. 
On the other hand, using the Harnack-type inequality (see (3.30)), we have that

$$
u_{l}(s \tilde{\theta}) \leq \bar{u}_{i, l}(s)+C \text { uniformly in } \tilde{\theta}
$$

hence we obtain

$$
\bar{u}_{i, l}(r) \leq C \int_{0}^{A} s^{3} \tilde{G}(r, s) e^{n \bar{u}_{i, l}(s)} d s-F_{i, l}^{\prime}(r)+H_{i, l}^{\prime}(r) .
$$

Now we study $\int_{0}^{A} s^{3} \tilde{G}(r, s) e^{4 \bar{u}_{i, l}(s)} d s$. To do so, let $R$ be so large such that $r_{i, l} \leq \frac{R_{i, l}}{4 R}$ (this is possible because of the assumption of contradiction). Now let us split the integral in the following way:

$$
\begin{aligned}
\int_{0}^{A} & s^{3} \tilde{G}(r, s) e^{4 \bar{u}_{i, l}(s)} d s \\
= & \int_{0}^{r_{i, l} / R} s^{3} \tilde{G}(r, s) e^{4 \bar{u}_{i, l}(s)} d s+\int_{r_{i, l} / R}^{r_{i, l} R} s^{3} \tilde{G}(r, s) e^{4 \bar{u}_{i, l}(s)} d s \\
& \quad+\int_{r_{i, l} R}^{R_{i, l} / C} s^{3} \tilde{G}(r, s) e^{4 \bar{u}_{i, l}(s)} d s+\int_{R_{i, l} / C}^{A} s^{3} \tilde{G}(r, s) e^{n \bar{u}_{i, l}(s)} d s
\end{aligned}
$$

Using the fact that we are at the scale $\frac{r_{i, l}}{R}$, then $\mathrm{d}$ of Step 1 implies that we have the following estimates for the first term of the equality above with $r=r_{i, l}$ :

$$
\int_{0}^{r_{i, l} / R} s^{3} \tilde{G}\left(r_{i, l}, s\right) e^{4 \bar{u}_{i, l}(s)} d s=-\frac{2}{r_{i, l}}+o_{l}(1) \frac{1}{r_{i, l}} .
$$

On the other hand, using assumption b of Step 1 we obtain the following estimates for the third term of the equality above with $r=r_{i, l}$ :

$$
\int_{r_{i, l} R}^{R_{i, l} / C} s^{3} \tilde{G}\left(r_{i, l}, s\right) e^{4 \bar{u}_{i, l}(s)} d s=o_{l}(1) \frac{1}{r_{i, l}} .
$$

We have also using assumption $d$ of Step 1 and the fact that $\frac{R_{i, l}}{r_{i, l}} \longrightarrow+\infty$ the following estimate for the fourth still with $r=r_{i, l}$ :

$$
\int_{R_{i, l} / C}^{A} s^{3} \tilde{G}\left(r_{i, l}, s\right) e^{4 \bar{u}_{i, l}(s)} d s=o_{l}(1) \frac{1}{r_{i, l}} .
$$

Now let us estimate the second term. For this, we will use point 3 of Step 3. First we recall that $r_{i, l}$ and $R$ verify the assumption of the latter. Hence 
the following holds

$$
\left\|r_{i, l}^{4} e^{4 \tilde{u}_{l}}\right\|_{C^{\alpha}\left(A_{R}\right)}=o_{l}(1)
$$

for the definition of $A_{R}$ and $\tilde{u}_{l}$ see statement of the point 3 of Step 3 where $d_{i, l}$ is replaced by $r_{i, l}$. On the other hand, performing a change of variable say $r_{i, l} y=s$, we obtain the following equality:

$$
\int_{r_{i, l} / R}^{r_{i, l} R} s^{3} \tilde{G}(r, s) e^{4 \bar{u}_{i, l}(s)} d s=\int_{1 / R}^{R} y^{3} \hat{G}_{i, l}(y) r_{i, l}^{4} e^{4 \hat{u}_{i, l}(y)} d y,
$$

where

$$
\begin{aligned}
\hat{u}_{i, l}(y) & =\bar{u}_{i, l}\left(r_{i, l} y\right), \\
\hat{G}_{i, l}(y) & =\tilde{G}\left(r_{i, l}, r_{i, l} y\right) .
\end{aligned}
$$

From the asymptotics of $\tilde{G}(\cdot, \cdot)$ (see $(3.50)$ ) we deduce the following one for $\hat{G}_{i, l}(\cdot, \cdot)$.

$$
\hat{G}_{i, l}(y)=\hat{f}_{i, l}(y) \frac{1}{r_{i, l}(1-y)}+\hat{H}_{i, l}(y)
$$

where $\hat{H}_{i, l}(\cdot)$ is integrable and $\hat{f}_{i, l}(\cdot)$ of class $C^{2}$.

Hence by using (3.52) and (3.53) we obtain the following inequality:

$$
\begin{aligned}
& \int_{r_{i, l} / R}^{r_{i, l} R} s^{3} \tilde{G}\left(r_{i, l}, s\right) e^{4 \bar{u}_{i, l}(s)} d s \\
& \quad=\frac{1}{r_{i, l}} \int_{1 / R}^{R} y^{3}\left(\frac{\hat{f}_{i, l}(y)}{(1-y)}+r_{i, l} \hat{H}_{i, l}(y)\right) r_{i, l}^{4} e^{4 \hat{u}_{i, l}(y)} d y .
\end{aligned}
$$

Moreover, using the Harnack-type inequality for $u_{l}$ (see (3.30) and (3.51)), we have that

$$
\left\|r_{i, l}^{4} e^{4 \hat{u}_{i, l}}\right\|_{C^{\alpha}(](1 / R), R[)}=o_{l}(1) .
$$

So using techniques of the theory of singular integral operators as in Lemma 4.4 [17] to have Holder estimates, we obtain

$$
\int_{1 / R}^{R} y^{3}\left(\frac{\hat{f}_{i, l}(y)}{(1-y)}+r_{i, l} \hat{H}_{i, l}(y)\right) r_{i, l}^{4} e^{4 \hat{u}_{i, l}(y)} d y=o_{l}(1)
$$


hence with (3.54) we deduce that

$$
\int_{r_{i, l} / R}^{r_{i, l} R} s^{3} \tilde{G}(r, s) e^{4 \bar{u}_{i, l}(s)} d s=o_{l}\left(\frac{1}{r_{i, l}}\right) .
$$

So we obtain

$$
\bar{u}_{i, l}^{\prime}\left(r_{i, l}\right) \leq-2 C \frac{1}{r_{i, l}}+o_{l}(1) \frac{1}{r_{i, l}}-F_{i, l}^{\prime}\left(r_{i, l}\right)+H_{i, l}^{\prime}(r) .
$$

Hence in both cases we get

$$
\bar{u}_{i, l}^{\prime}\left(r_{i, l}\right) \leq-2 C \frac{1}{r_{i, l}}+o_{l}(1) \frac{1}{r_{i, l}}-F_{i, l}^{\prime}\left(r_{i, l}\right)+H_{i, l}^{\prime}(r) .
$$

Now let compute $\varphi_{i, l}^{\prime}\left(r_{i, l}\right)$. From straightforward computations, we have

$$
\varphi_{i, l}^{\prime}\left(r_{i, l}\right)=\left(r_{i, l}\right)^{4 \nu-1} \exp \left(\bar{u}_{i, l}\left(r_{i, l}\right)\right)\left(4 \nu+r_{i, l} \bar{u}_{i, l}^{\prime}\left(r_{i, l}\right)\right) .
$$

So using (3.56) we arrive to the following inequality:

$$
\begin{aligned}
& \varphi_{i, l}^{\prime}\left(r_{i, l}\right) \\
& \quad \leq\left(r_{i, l}\right)^{4 \nu-1} \exp \left(\bar{u}_{i, l}\left(r_{i, l}\right)\left(4 \nu-2 C+o_{l}(1)-r_{i, l} F_{i, l}^{\prime}\left(r_{i, l}\right)+r_{i, l} H_{i, l}^{\prime}\left(r_{i, l}\right)\right) ;\right.
\end{aligned}
$$

so $\nu<\frac{1}{2}$ implies $4 \nu-2 C+o_{l}(1)<0$ for $l$ sufficiently large.

Thus since $F_{i, l}^{\prime}$ and $H_{i, l}^{\prime}$ are bounded in $\left(0, \frac{\operatorname{inj}_{g}(M)}{4}\right)$ uniformly in $l$ and $r_{i, l} \longrightarrow 0$, we have that for $l$ big enough

$$
\varphi_{i, l}^{\prime}\left(r_{i, l}\right)<0
$$

hence we reach the desired contradiction and conclude the proof of the step.

Step 5 (Proof of Theorem 1.4). We show first the following estimate

$$
\int_{M \backslash \cup_{i=1}^{i=N} B_{x_{i, l}}\left(R_{i, l} / C\right)} e^{4 u_{l}(y)} d V_{g}(y)=o_{l}(1) .
$$

For this, we first start by proving

$$
\bar{u}_{l} \longrightarrow-\infty \text { as } l \longrightarrow+\infty \text {. }
$$


In fact, using Green's representation formula for $u_{l}$ (see Lemma 2.3), we have that for every $x \in M$

$$
\begin{aligned}
u_{l}(x)= & \bar{u}_{l}+2 \int_{M} G(x, y)\left(\bar{Q}(y) e^{4 u_{l}(y)}-Q_{l}(y)\right) d V_{g}(y) \\
& +-\int_{M} G\left(x, y^{\prime}\right) T_{l}\left(y^{\prime}\right) d S_{g}\left(y^{\prime}\right) \\
\geq & \bar{u}_{l}-C+2 \int_{M} G(x, y) \bar{Q}(y) e^{4 u_{l}(y)} d V_{g}(y)
\end{aligned}
$$

By assumption c or d of Step 1 we have given any $\epsilon>0$, there exists $R_{\epsilon}$ such that for $l$ sufficiently large if $\bar{x}_{i} \in \operatorname{int}(M)$, then

$$
\int_{B_{x_{1, l}}\left(R_{\epsilon} \mu_{1, l}\right)} \bar{Q}_{l}(y) e^{4 u_{l}(y)} d V_{g}(y) \geq 8 \pi^{2}-\frac{\epsilon}{32 \pi^{2}}
$$

and if $\bar{x}_{i} \in \partial M$, then

$$
\int_{B_{x_{1}, l}^{+}\left(R_{\epsilon} \mu_{1, l}\right)} \bar{Q}_{l}(y) e^{4 u_{l}(y)} d V_{g}(y) \geq 4 \pi^{2}-\frac{\epsilon}{16 \pi^{2}} .
$$

Hence the last three formulas and the asymptotics of Green's function of $\left(P_{g}^{4}, P_{g}^{3}\right)$ imply that if $\bar{x}_{1} \in \operatorname{int}(M)$, then

$$
e^{4 u_{l}}(x) \geq C^{-1} e^{4 \bar{u}_{l}} \frac{1}{\left|x-x_{1, l}\right|^{8-\epsilon}} \text { for }\left|x-x_{1, l}\right| \geq 2 R_{\epsilon} \mu_{1, l} \quad \text { for } l \text { large, }
$$

and if $\bar{x}_{1} \in \partial M$, then

$$
e^{4 u_{l}}(x) \geq C^{-1} e^{4 \bar{u}_{l}} \frac{1}{\left|x-x_{1, l}\right|^{8-\epsilon}} \text { for }\left|x-x_{1, l}\right| \geq 2 R_{\epsilon} \mu_{1, l} \quad \text { for } l \text { large. }
$$

From this it follows that

$$
\begin{aligned}
\int_{M} e^{4 u_{l}(y)} d V_{g}(y) & \geq \int_{\left(B_{x_{1, l}}^{+}\left(\operatorname{inj}_{g}(M)\right) \backslash B_{x_{1, l}}^{+}\left(2 R_{\epsilon} \mu_{1, l}\right)\right)} e^{4 u_{l}(y)} d V_{g}(y) \\
& \geq C^{-1} e^{4 \bar{u}_{l}} \int_{2 R_{\epsilon} \mu_{1, l}}^{\operatorname{inj}_{g}(M)} s^{\epsilon-(5)} d s \geq C^{-1} e^{4 \bar{u}_{l}}\left(2 R_{\epsilon} \mu_{1, l}\right)^{\epsilon-4} .
\end{aligned}
$$

So if $\epsilon$ is small enough, we have from (1.10) that

$$
\bar{u}_{l} \longrightarrow-\infty
$$

hence we are done. 
Now by assumption b of Step 1 , we can cover $M \backslash \cup_{i=1}^{i=N} B_{x_{i, l}}\left(\frac{R_{i, l}}{C}\right)$ with a finite number of balls $B_{y_{k}}\left(r_{k}\right)$ such that for any $k$ there holds

$$
\int_{B_{y_{k}}^{+}\left(2 r_{k}\right)} \bar{Q}_{l} e^{4 u_{l}(y)} d V_{g}(y) \leq 4 \pi^{2}
$$

Now set $B_{k}=B_{y_{k}}\left(2 r_{k}\right)$ and $\tilde{B}_{k}=B_{y_{k}}\left(r_{k}\right)$, so using again the Green representation formula for $u_{l}$ we have $\forall x \in \tilde{B}_{k}$

$$
\begin{aligned}
u_{l}(x)= & \bar{u}_{l}+2 \int_{M} G(x, y) \bar{Q}_{l} e^{4 u_{l}(y)} d V_{g}(y)-\int_{M} G(x, y) Q_{l}(y) d V_{g}(y) \\
& -\int_{\partial M} G\left(x, y^{\prime}\right) T_{l}\left(y^{\prime}\right) d S_{g}\left(y^{\prime}\right)
\end{aligned}
$$

hence

$$
\begin{aligned}
u_{l}(x) \leq & \bar{u}_{l}+C+2 \int_{M} G(x, y) \bar{Q}_{l} e^{4 u_{l}(y)} d V_{g}(y) \\
= & \bar{u}_{l}+C+2 \int_{B_{k}} G(x, y) \bar{Q}_{l} e^{4 u_{l}(y)} d V_{g}(y) \\
& +2 \int_{M \backslash B_{k}} G(x, y) \bar{Q}_{l} e^{4 u_{l}(y)} d V_{g}(y) .
\end{aligned}
$$

So since $G$ is smooth out of the diagonal, we have that

$$
u_{l}(x) \leq \bar{u}_{l}+C+2 \int_{B_{k}} G(x, y) \bar{Q}_{l}(y) e^{n u_{l}(y)} d V_{g}(y)
$$

Now using Jensen's inequality, we obtain

$$
\begin{aligned}
& \exp \left(\int_{B_{k}} G(x, y) \bar{Q}_{l} e^{4 u_{l}(y)} d V_{g}(y)\right) \\
& \quad \leq \int_{M} \exp \left(\left\|\bar{Q} e^{4 u_{l}} \chi_{B_{k}}\right\|_{L^{1}(M)}|G(x, y)|\right) \frac{\bar{Q}_{l}(y) e^{4 u_{l}(y)} \chi_{B_{k}}(y)}{\left\|\bar{Q} e^{4 u_{l}} \chi_{B_{k}}\right\|_{L^{1}(M)}} d V_{g}(y) .
\end{aligned}
$$

Hence using the Fubini theorem, we have

$$
\int_{\tilde{B}_{k}} e^{4 u_{l}}(y) d v_{g}(x) \leq C e^{4 \bar{u}_{l}} \sup _{y \in M, k} \int_{M}\left(\frac{1}{d_{g}(x, y)}\right)^{1 / 2 \pi^{2}\left\|\bar{Q} e^{4 u_{l}} \chi_{B_{k}}\right\|_{L^{1}(M)}} d V_{g}(x)
$$


So from $\int_{B_{k}} \bar{Q}_{l}(y) e^{4 u_{l}(y)} d V_{g}(y) \leq 4 \pi^{2}$ and (3.58), we have that

$$
\int_{\tilde{B}_{k}} e^{4 u_{l}(y)} d V_{g}(y)=o_{l}(1) \quad \forall k
$$

Hence

$$
\int_{M \backslash \cup_{i=1}^{i=N} B_{x_{i, l}}\left(R_{i, l} / C\right)} e^{4 u_{l}(y)} d V_{g}(y)=o_{l}(1) .
$$

So since $B_{x_{i, l}}\left(\frac{R_{i, l}}{C}\right)$ are disjoint, then Step 4 implies that

$$
\int_{M} \bar{Q}_{l}(y) e^{4 u_{l}(y)} d V_{g}(y)=4 N \pi^{2}+o_{l}(1),
$$

hence (1.10) implies that

$$
\int_{M} Q_{0}(y) d V_{g}(y)+\int_{\partial M} T_{0}\left(y^{\prime}\right) d S_{g}\left(y^{\prime}\right)=4 N \pi^{2},
$$

ending the proof of Theorem 1.4.

\section{Proof of Theorem 1.1}

This section deals with the proof of Theorem 1.1. It is divided into four subsections. The first one is concerned with an improvement of the MoserTrudinger-type inequality (see Proposition 2.7) and its corollaries. The second one is about the existence of a non-trivial global projection from some negative sublevels of II onto $\left(M_{\partial}\right)_{k}$ (for the definition, see Section 2 , formula 2.2). The third one deals with the construction of a map from $\left(M_{\partial}\right)_{k}$ into suitable negative sublevels of II. The last one describes the min-max scheme.

\subsection{Improved Moser-Trudinger inequality}

In this subsection, we give an improvement of the Moser-Trudinger-type inequality, see Proposition 2.7. Afterwards, we state a lemma which gives some sufficient conditions for the improvement to hold (see (4.13) to (4.16)). By these results, we derive a relation between the number of possible interior and boundary concentration points for $e^{4 u}$ present at the same time with $k$ 
given as in (1.6) where $u \in H_{\partial / \partial n}$ is an arbitrary function for which $\operatorname{II}(u)$ attains large negative values (see Lemma 4.3). Finally from these results, we derive a corollary which gives the distance of $e^{4 u}$ (for some functions $u$ suitably normalized) from $\left(M_{\partial}\right)_{k}$.

As said in the introduction of the subsection, we start by the following Lemma giving an improvement of the Moser-Trudinger-type inequality (Proposition 2.7).

Lemma 4.1. For a fixed $l_{1}, l_{2} \in \mathbb{N}, l_{1}+l_{2} \neq 0$ and $\delta>0$, let $S_{1} \cdots S_{l_{1}}$, $\Omega_{1} \cdots \Omega_{l_{2}}$ be subsets of $M$ satisfying $\bar{S}_{i} \subset \subset M_{\delta}$, $\operatorname{dist}\left(S_{i}, S_{j}\right) \geq \delta_{0}$ for $i \neq j$, $\operatorname{dist}\left(\Omega_{i}, \Omega_{j}\right) \geq \delta_{0}, \Omega_{i} \cap \partial M \neq \emptyset, \bar{\Omega}_{i} \subset \subset \partial M \times[0, \delta]$ and let $\gamma_{0} \in\left(0, \frac{1}{l_{1}+l_{2}}\right)$.

Then, for any $\bar{\epsilon}>0$, there exists a constant $C=C\left(\bar{\epsilon}, \delta_{0}, \gamma_{0}, l_{1}, l_{2}, M, \delta\right)$ such that the following holds:

$$
\log \int_{M} e^{4(u-\bar{u})} \leq C+\frac{1}{4 \pi^{2}}\left(\frac{1}{2 l_{1}+l_{2}-\bar{\epsilon}}\right)\left\langle P_{g}^{4,3} u, u\right\rangle_{L^{2}(M)},
$$

for all the functions $u \in H_{\partial / \partial n}$ satisfying

$$
\frac{\int_{S_{i}} e^{4 u} d V g}{\int_{M} e^{4 u} d V g} \geq \gamma_{0}, \quad i \in\left\{1, \ldots, l_{1}\right\}
$$

and

$$
\frac{\int_{\Omega_{i}} e^{4 u} d V g}{\int_{M} e^{4 u} d V g} \geq \gamma_{0}, \quad i \in\left\{1, \ldots, l_{2}\right\} .
$$

Proof. We modify the argument in [4] and [14]. First of all we can assume without loss of generality that $\bar{u}=0$. On the other hand, by the properties verified by the sets $S_{i}$ and $\Omega_{i}$ we have that there exists

$N_{\delta_{0}, \delta} \subset M$ closed submanifold of dimension $4, \overline{\cup S_{i}} \subset \subset N_{\delta_{0}, \delta} \subset \operatorname{int}(M)$,

$$
\overline{\cup \Omega_{i}} \subset \subset \backslash N_{\delta_{0}, \delta} .
$$


We can find $l_{1}+l_{2}$ functions $g_{1}, \ldots, g_{l_{1}}$ and $h_{1}, \ldots, h_{l_{2}}$ such that

$$
\left\{\begin{array}{l}
g_{i}(x) \in[0,1] \quad \text { for every } x \in M, i=1, \ldots, l_{1}, \\
g_{i}(x)=1 \quad \text { for } x \in S_{i}, i=1, \ldots, l_{1}, \\
g_{i}(x)=0 \quad \text { if } \operatorname{dist}\left(x, S_{i}\right) \geq \frac{\delta_{0}}{4}, i=1, \ldots, l_{1}, \\
\operatorname{supp}\left(g_{i}\right) \subset N_{\delta_{0}, \delta} \\
\left\|g_{i}\right\|_{C^{4}(M)}, \leq C_{\delta_{0}, \delta} \quad \text { for } i=1, \ldots, l_{1}, \\
\sum_{i=1}^{l_{1}} g_{i}=1 \text { on } N_{\delta_{0}, \delta}
\end{array}\right.
$$

and

$$
\left\{\begin{array}{l}
h_{i}(x) \in[0,1] \quad \text { for every } x \in M, i=1, \ldots, l_{2}, \\
h_{i}(x)=1 \quad \text { for } x \in \Omega_{i}, i=1, \ldots, l_{2}, \\
h_{i}(x)=0 \quad \text { if } \operatorname{dist}\left(x, \Omega_{i}\right) \geq \frac{\delta_{0}}{4}, i=1, \ldots, l_{2}, \\
\left\|h_{i}\right\|_{C^{4}(M)}, \leq C_{\delta_{0}} \quad \text { for } i=1, \ldots, l_{2}, \\
\sum_{i=1}^{l_{2}} h_{i}=1 \text { on } M \backslash N_{\delta_{0}, \delta},
\end{array}\right.
$$

where $C_{\delta_{0}, \delta}$ is a positive constant depending only on $\delta_{0}$ and $\delta$. Moreover, we can choose the functions $g_{i}$ and $h_{i}$ such that they have (mutually) disjoint supports.

We remark that the submanifold $N_{\delta_{0}, \delta}$ depends only on $\delta_{0}$ and $\delta$. But since in our analysis only its volume is involved, when we apply the MoserTrudinger inequality to $g_{i} u_{2}$, see (4.9), then (thanks to Remark 2.1) we can omit the dependence to $\delta_{0}$ and $\delta$.

Using the Leibniz rule, Schwartz inequality and interpolation, we obtain that for every $\epsilon>0$ there exists $C_{\epsilon, \delta_{0}, \delta}$ (depending only on $\epsilon, \delta$ and $\delta_{0}$ ) such that $\forall v \in H^{2}(M)$, for any $i=1, \ldots, l_{1}$ and $j=1, \ldots, l_{2}$ there holds

$$
\left\langle P_{g}^{4,3} g_{i} v, g_{i} v\right\rangle \leq \int_{M} g_{i}^{2}\left(P_{g}^{4,3} v, v\right) d V_{g}+\epsilon\left\langle P_{g}^{4,3} v, v\right\rangle_{L^{2}(M)}+C_{\epsilon, \delta_{0}, \delta} \int_{M} v^{2} d V_{g}
$$

and

$$
\left\langle P_{g}^{4,3} h_{j} v, h_{j} v\right\rangle \leq \int_{M} h_{j}^{2}\left(P_{g}^{4} v, v\right) d V_{g}+\epsilon\left\langle P_{g}^{4,3} v, v\right\rangle_{L^{2}(M)}+C_{\epsilon, \delta_{0}, \delta} \int_{M} v^{2} d V_{g}
$$


Next we decompose $u$ in Fourier mode, namely we set $u=u_{1}+u_{2}$ with $u_{1} \in L^{\infty}(M)$. Hence from our assumptions, see (4.1) and (4.2), we derive that

$$
\int_{S_{i}} e^{4 u_{2}} d V_{g} \geq e^{-4\left\|u_{1}\right\|_{L^{\infty}}} \gamma_{0} \int_{M} e^{4 u} d V_{g}, \quad i=1, \ldots, l_{1},
$$

and

$$
\int_{\Omega_{i}} e^{4 u_{2}} d V_{g} \geq e^{-4\left\|u_{1}\right\|_{L^{\infty}}} \gamma_{0} \int_{M} e^{4 u} d V_{g}, \quad i=1, \ldots, l_{2} .
$$

Now using (4.7), (4.8) and the trivial identity

$$
\log \int_{M} e^{4 u} d V_{g}=\frac{2 l_{1}}{2 l_{1}+l_{2}} \log \int_{M} e^{4 u} d V_{g}+\frac{l_{1}}{2 l_{1}+l 2} \log \int_{M} e^{4 u} d V_{g},
$$

we obtain

$$
\begin{aligned}
\log \int_{M} e^{4 u} d V_{g} \leq & \log \frac{1}{\gamma_{0}}+4\left\|u_{1}\right\|_{L^{\infty}}+\frac{2 l_{1}}{2 l_{1}+l_{2}} \log \int_{N} e^{g_{i} 4 u_{2}} \\
& +\frac{l_{2}}{2 l_{1}+l_{2}} \log \int_{M} e^{4 h_{j} u_{2}} d V_{g}+C
\end{aligned}
$$

where $C$ depends only on $M$. On the other hand, by using Lemma 2.1 we get

$$
\log \int_{N} e^{g_{i} 4 u_{2}} \leq C_{M}+\frac{1}{8 \pi^{2}}\left\langle P_{g, N}^{4}\left(g_{i} u_{2}\right), g_{i} u_{2}\right\rangle+4 \overline{g_{i} u_{2}},
$$

where $P_{g, N}^{4}$ denotes the Paneitz operator associated to the close 4-manifold $N$ endowed with the induced metric from $g$, and $C_{M}$ depends only on $\operatorname{Vol}_{g}(M)$.

Now let $\alpha<16 \pi^{2}$ (to be fixed later), from Proposition 2.7 we infer

$$
\begin{aligned}
\log \int_{M} e^{4 u} d V_{g} \leq & \log \frac{1}{\gamma_{0}}+4\left\|u_{1}\right\|_{L^{\infty}}+\frac{4}{2 \alpha}\left(\frac{2 l_{1}}{2 l_{1}+l_{2}}\right)\left\langle P_{g, N}^{4}\left(g_{i} u_{2}\right), g_{i} u_{2}\right\rangle \\
& +\frac{4}{\alpha}\left(\frac{l_{2}}{2 l_{1}+l_{2}}\right)\left\langle P_{g}^{4,3}\left(h_{j} u_{2}\right), h_{j} u_{2}\right\rangle \\
& +4\left(\frac{2 l_{1}}{2 l_{1}+l_{2}}\right) \overline{g_{i} u_{2}}+4\left(\frac{l_{2}}{2 l_{1}+l_{2}}\right) \overline{h_{j} u_{2}}+C_{\alpha, M, l_{1}, l_{2}},
\end{aligned}
$$


where $C_{\alpha, M, l_{1}, l_{2}}$ depends only on $\alpha, l_{1}, l_{2}$ and $M$. We now choose $i$ and $j$ such that

$$
\int_{N} g_{i}^{2}\left(P_{g, N}^{4} u_{2}, u_{2}\right) d V_{g} \leq \int_{N} g_{p}^{2}\left(P_{g, N}^{4} u_{2}, u_{2}\right) d V_{g} \quad \text { for every } p=1, \ldots, l_{1},
$$

and

$$
\int_{M} h_{j}^{2}\left(P^{4}, 3 g u_{2}, u_{2}\right) d V_{g} \leq \int_{M} h_{q}^{2}\left(P_{g}^{4,3} u_{2}, u_{2}\right) d V_{g} \quad \text { for every } q=1, \ldots, l_{2} .
$$

Hence since the functions $g_{p}, h_{q}$ have disjoint supports and verify (4.3) and (4.4), then by (4.5), (4.6) and (4.10), we get

$$
\begin{aligned}
& \log \int_{M} e^{4 u} d V_{g} \\
& \quad \leq \log \frac{1}{\gamma_{0}}+4\left\|u_{1}\right\|_{L^{\infty}}+\frac{4}{\alpha}\left(\frac{1}{2 l_{1}+l_{2}}+\epsilon\right)\left\langle P_{g}^{4,3} u_{2}, u_{2}\right\rangle+C_{\epsilon, \delta_{0}} \int_{M} u_{2}^{2} d V_{g}
\end{aligned}
$$

$$
+4\left(\frac{2 l_{1}}{2 l_{1}+l_{2}}\right) \overline{g_{i} u_{2}}+4\left(\frac{l_{2}}{2 l_{1}+l_{2}}\right) \overline{h_{j} u_{2}}+C_{\alpha, M, l_{1}, l_{2}} .
$$

Now we choose $\lambda_{\epsilon, \delta_{0}, \delta}$ to be an eigenvalue of $P_{g}^{4,3}$ such that $\frac{C_{\epsilon, \delta_{0}, \delta}}{\lambda_{\epsilon, \delta_{0}, \delta}}<\epsilon$ and we set

$$
u_{1}=P_{V_{\epsilon, \delta_{0}, \delta}} u, \quad u_{2}=P_{V_{\epsilon, \delta_{0}, \delta}^{\perp}} u
$$

where $V_{\epsilon, \delta_{0}, \delta}$ is the direct sum of the eigenspaces of $P_{g}^{4,3}$ with eigenvalues less or equal to $\lambda_{\epsilon, \delta_{0}, \delta}$, and $P_{V_{\epsilon, \delta_{0}, \delta}}, P_{V_{\epsilon, \delta_{0}, \delta}^{\perp}}$ denote the projections onto $V_{\epsilon, \delta_{0}, \delta}$ and $V_{\epsilon, \delta_{0}, \delta}^{\perp}$, respectively. Since $\bar{u}=0$, then the $L^{2}$-norm and the $L^{\infty}$-norm on $V_{\epsilon, \delta_{0}, \delta}$ are equivalent (with a proportionality factor which depends on $\epsilon$, $\delta$ and $\left.\delta_{0}\right)$. Hence by the choice of $u_{1}$ and $u_{2}$, see (4.12), we have that

$$
\left\|u_{1}\right\|_{L^{\infty}} \leq \tilde{C}_{\epsilon, \delta_{0}, \delta}\left\langle P_{g}^{4,3} u_{1}, u_{1}\right\rangle^{1 / 2}
$$

and

$$
C_{\epsilon, \delta_{0}, \delta} \int_{M} u_{2}^{2} d V_{g}<\epsilon\left\langle P_{g}^{4,3} u_{2}, u_{2}\right\rangle
$$


where $\tilde{C}_{\epsilon, \delta_{0}, \delta}$ depends on $\epsilon, \delta$ and $\delta_{0}$. Furthermore, by the Hólder inequality and Lemma 2.9, we have that

$$
\overline{g_{i} u_{2}} \leq C\left\langle P_{g}^{4,3} u, u\right\rangle^{1 / 2}
$$

and

$$
\overline{h_{j} u_{2}} \leq C\left\langle P_{g}^{4,3} u, u\right\rangle^{1 / 2}
$$

So (4.11) becomes

$$
\begin{aligned}
\log \int_{M} e^{4 u} d V_{g} \leq & 2 \log \frac{1}{\gamma_{0}}+\hat{C}_{\epsilon, \delta_{0}, \delta}\left\langle P_{g}^{4,3} u_{1}, u_{1}\right\rangle^{1 / 2} \\
& +\frac{4}{\alpha}\left(\frac{1}{2 l_{1}+l_{2}}+\epsilon\right)\left\langle P_{g}^{4,3} u_{2}, u_{2}\right\rangle+\epsilon\left\langle P_{g}^{4,3} u_{2}, u_{2}\right\rangle \\
& +C_{l_{1}, l_{2}}\left\langle P_{g}^{4,3} u, u\right\rangle^{1 / 2}+C_{\alpha, M, l-1, l_{2}}
\end{aligned}
$$

where $\hat{C}_{\epsilon, \delta_{0}, \delta}=4 \tilde{C}_{\epsilon, \delta_{0}, \delta}$. Thus by using the Cauchy inequality, we get

$$
\log \int_{M} e^{4 u} d V_{g} \leq C_{\epsilon, \delta_{0}, \gamma_{0}, \alpha, l_{1}, l_{2}, M}+\frac{4}{\alpha}\left(\frac{1}{2 l_{1}+l_{2}}+3 \epsilon\right)\left\langle P_{g}^{4,3} u_{2}, u_{2}\right\rangle .
$$

Now setting $\alpha=16 \pi^{2}-4 \epsilon$, we obtain

$$
\log \int_{M} e^{4 u} d V_{g} \leq C_{\epsilon, \delta_{0}, \gamma_{0}, l_{1}, l_{2}, M}+\frac{1}{4 \pi^{2}-\epsilon}\left(\frac{1}{2 l_{1}+l_{2}}+3 \epsilon\right)\left\langle P_{g}^{4,3} u_{2}, u_{2}\right\rangle .
$$

So choosing $\epsilon$ such that $\frac{1}{4 \pi^{2}-\epsilon}\left(\frac{1}{2 l_{1}+l_{2}}+3 \epsilon\right) \leq \frac{1}{4 \pi^{2}}\left(\frac{1}{2 l_{1}+l_{2}-\bar{\epsilon}}\right)$, we get

$$
\log \int_{M} e^{4 u} d V_{g} \leq C_{\epsilon, \delta_{0}, \gamma_{0}, l_{1}, l_{2}, M}+\frac{1}{4 \pi^{2}}\left(\frac{1}{2 l_{1}+l_{2}-\bar{\epsilon}}\right)\left\langle P_{g}^{4,3} u_{2}, u_{2}\right\rangle .
$$

Hence the lemma is proved.

In the next Lemma we show a criterion which implies the situation described in the conditions in (4.1) and (4.2). The proof is a trivial adaptation of the arguments of Lemma 2.3 in [14].

Lemma 4.2. Let $h$ and $l$ be positive integer, and suppose that $\epsilon, r$ and $\delta$ are positive numbers. Assume $f \in L^{1}(M)$ is a non-negative function such that $\|f\|_{L^{1}(M)}=1$, then we have the following: 
1) If $\int_{M \backslash M_{4 \delta}} f d V_{g}<\epsilon$, then there holds If

$\int_{M_{4 \delta} \cap\left(\cup_{i=1}^{h} B_{p_{i}}(r)\right)} f d V_{g}<\int_{M_{4 \delta}} f d V_{g}-$ efor every $h$-tuples $p_{1}, \ldots, p_{h}$

$$
\in M_{4 \delta} \text { such that } B_{p_{i}}(2 r) \subset M_{2 \delta} \text {, }
$$

then there exist $\bar{\varepsilon}>0$ and $\bar{r}>0$, depending only on $\varepsilon, r, \tilde{h}, \delta$ and $M$ (but not on $f$ ), and points $\bar{p}_{1}, \ldots, \bar{p}_{h+1} \in M_{4 \delta}$, satisfying

$$
\begin{gathered}
\int_{B_{\bar{p}_{1}}(\bar{r})} f d V_{g}>\bar{\varepsilon}, \ldots, \int_{B_{\bar{p}_{\tilde{h}}}(\bar{r})} f d V_{g}>\bar{\varepsilon} ; \quad B_{\bar{p}_{i}}(2 \bar{r}) \cap B_{\bar{p}_{j}}(2 \bar{r})=\emptyset \\
\text { for } i \neq j, B_{\bar{p}_{j}}(2 \bar{r}) \subset M_{2 \delta} .
\end{gathered}
$$

2) If $\int_{M_{\delta / 4}} f d V_{g}<\epsilon$, then there holds If

$$
\begin{gathered}
\int_{\partial M \times\left[0, \delta / 4\left[\cap\left(\cup_{i=1}^{h} B_{q_{i}}^{+}(r)\right)\right.\right.} f d V_{g}<\int_{\partial M \times[0, \delta / 4]} f d V_{g}-\varepsilon \\
\text { for every l-tuples } q_{1}, \ldots, q_{l} \in \partial M, \quad B_{q_{j}}^{+}(2 r) \subset \partial M \times\left[0, \frac{\delta}{2}\right],
\end{gathered}
$$

then there exist $\bar{\varepsilon}>0$ and $\bar{r}>0$ depending only on $\varepsilon, r, \tilde{l}$ and $M$ (but not on $f)$, and points $\bar{q}_{1}, \ldots, \bar{q}_{l+1} \in \partial M, B_{\bar{q}_{j}}^{+}(2 r) \subset \partial M \times\left[0, \frac{\delta}{2}\right]$ satisfying

$$
\int_{B_{\bar{q}_{1}}^{+}(\bar{r})} f d V_{g}>\bar{\varepsilon}, \ldots, \int_{B_{\bar{q}_{\bar{l}}}^{+}(\bar{r})} f d V_{g}>\bar{\varepsilon} ; \quad B_{\bar{q}_{i}}^{+}(2 \bar{r}) \cap B_{\bar{q}_{j}}^{+}(2 \bar{r})=\emptyset \text { for } i \neq j .
$$

3) If $\int_{M \backslash M_{4 \delta}} f d V_{g} \geq \epsilon$ and $\int_{M_{\delta / 4}} f d V_{g} \geq \epsilon$, then there holds

$$
\begin{aligned}
& \int_{M_{4 \delta} \cap\left(\cup_{i=1}^{h} B_{p_{i}}(r)\right)} f d V_{g}<\int_{M_{4 \delta}} f d V_{g}-\varepsilon \text { for every h-tuples } p_{1}, \ldots, p_{h} \in M_{4 \delta} \\
& \text { such that } B_{p_{i}}(2 r) \subset M_{2 \delta}
\end{aligned}
$$

and

$$
\begin{gathered}
\int_{\partial M \times\left[0, \delta / 4\left[\cap\left(\cup_{i=1}^{h} B_{q_{i}}^{+}(r)\right)\right.\right.} f d V_{g}<\int_{\partial M \times[0, \delta / 4]} f d V_{g}-\varepsilon \\
\text { for every l-tuples } q_{1}, \ldots, q_{l} \in \partial M, \quad B_{q_{j}}^{+}(2 r) \subset \partial M \times\left[0, \frac{\delta}{2}\right]
\end{gathered}
$$


then there exist $\bar{\varepsilon}>0$ and $\bar{r}>0$, depending only on $\varepsilon, r, \tilde{h}, l, \delta$ and $M$ (but not on $f$ ), points $\bar{p}_{1}, \ldots, \bar{p}_{h+1} \in, M_{4 \delta}$ and points $\bar{q}_{1}, \ldots, \bar{q}_{l+1} \in$ $\partial M, B_{\bar{q}_{j}}^{+}(2 r) \subset \partial M \times\left[0, \frac{\delta}{2}\right]$ satisfying

$$
\begin{gathered}
\int_{B_{\bar{p}_{1}}(\bar{r})} f d V_{g}>\bar{\varepsilon}, \ldots, \int_{B_{\bar{p} \tilde{h}}(\bar{r})} f d V_{g}>\bar{\varepsilon} \\
B_{\bar{p}_{i}}(2 \bar{r}) \cap B_{\bar{p}_{j}}(2 \bar{r})=\emptyset \quad \text { for } i \neq j, B_{\bar{p}_{j}}(2 \bar{r}) \subset M_{2 \delta} .
\end{gathered}
$$

and

$$
\int_{B_{\bar{q}_{1}}^{+}(\bar{r})} f d V_{g}>\bar{\varepsilon}, \ldots, \int_{B_{\bar{q}_{\bar{l}}}^{+}(\bar{r})} f d V_{g}>\bar{\varepsilon} ; \quad B_{\bar{q}_{i}}^{+}(2 \bar{r}) \cap B_{\bar{q}_{j}}^{+}(2 \bar{r})=\emptyset \text { for } i \neq j .
$$

The next lemma is an interesting consequence of Lemma 4.1. It characterizes some functions in $H_{\partial / \partial n}$ for which the value of II is large negative.

Lemma 4.3. Under the assumptions of Theorem 1.1, and for $k \geq 1$ given by (1.6), the following property holds. For any $\epsilon>0$ and $r>0$ (all small), there exists large positive $L=L(\epsilon, r)$ such that for any $u \in H_{\partial / \partial n}$ with $\mathrm{II}(u) \leq-L, \int_{M} e^{4 u} d V_{g}=1$ the following holds, $\forall \delta>0$ (small) there the following holds:

1) If $\int_{M \backslash M_{4 \delta}} e^{4 u} d V_{g}<\epsilon$, then there exists $\tilde{k}$ points $p_{1, u}, \ldots, p_{\tilde{k}, u} \in M_{4 \delta}$, $B_{p_{i, u}}(2 r) \subset M_{2 \delta}$ such that

$$
\int_{M_{4 \delta} \backslash \cup_{i=1}^{h} B_{p_{i, u}}(r)} e^{4 u} d V_{g}<\epsilon
$$

2) If $\int_{M_{\delta / 4}} e^{4 u} d V g<\epsilon$, then there exists $k$ points $q_{1, u}, \ldots, q_{k, u} \in \partial M$, $B_{q_{i, u}}^{+}(2 r) \subset \partial M \times\left[0, \frac{\delta}{2}\right]$ such that

$$
\int_{\partial M \times[0, \delta / 4] \backslash \cup_{i=1}^{l} B_{q_{i, u}}^{+}(r)} e^{4 u} d V_{g}<\epsilon
$$

If $\int_{M \backslash M_{4 \delta}} e^{4 u} d V_{g} \geq \epsilon$ and $\int_{M_{\delta / 4}} e^{4 u} d V g \geq \epsilon$, then there exists $(h, l) \in$ $\mathbb{N}^{*} \times \mathbb{N}^{*}, 2 h+l \leq k, h$ points $p_{1, u}, \ldots, p_{h, u} \in M_{4 \delta}, B_{p_{i, u}}(2 r) \subset M_{2 \delta}$ 
and $l$ points $q_{1, u}, \ldots, q_{l, u} \in \partial M, B_{q_{i, u}}^{+}(2 r) \subset \partial M \times\left[0, \frac{\delta}{2}\right]$ such that

$$
\int_{M_{4 \delta} \backslash \cup_{i=1}^{h} B_{p_{i, u}}(r)} e^{4 u} d V_{g}<\epsilon
$$

and

$$
\int_{\partial M \times[0, \delta / 4] \backslash \cup_{i=1}^{l} B_{q_{i, u}}^{+}(r)} e^{4 u} d V_{g}<\epsilon .
$$

Proof. Suppose that by contradiction the statement is not true. Then there exists $\epsilon>0, r>0, \delta>0$ and a sequence $\left(u_{n}\right) \in H_{\partial n}$ such that $\int_{M} e^{4 u_{n}}$ $d V_{g}=1, \mathrm{II}\left(u_{n}\right) \rightarrow-\infty$ as $n \rightarrow+\infty$ and such that either

1) $\int_{M \backslash M_{4 \delta}} e^{4 u_{n}} d V_{g}<\epsilon$ and $\tilde{k}$ tuples of points $p_{1}, \ldots, p_{k} \in M_{4 \delta}$ and $B_{p_{i}}(2 r)$ $\subset M_{2 \delta}$, we have

$$
\int_{M_{4 \delta} \cap\left(\cup_{i=1}^{h} B_{p_{i, u}}(r)\right)} e^{4 u} d V_{g}<\int_{M_{4 \delta}} f d V_{g}-\epsilon
$$

2) $\int_{M_{\delta / 4}} e^{4 u_{n}} d V_{g}<\epsilon$ and $\forall k$ tuples of points $q_{1}, \ldots, q_{k} \in \partial M$, we have

$$
\text { or } \int_{\partial M \times\left[0, \delta / 4 \cap\left(\cup_{i=1}^{l} B_{q_{i, u}}^{+}(r)\right)\right.} e^{4 u} d V_{g}<\int_{\partial M \times[0, \delta / 4]} f d V_{g}-\epsilon
$$

3) $\int_{M \backslash M_{4 \delta}} e^{4 u_{n}} d V_{g} \geq \epsilon, \int_{M_{\delta / 4}} e^{4 u_{n}} d V_{g} \geq \epsilon$ and $\forall(h, l) \in \mathbb{N}^{*} \times \mathbb{N}^{*}, 2 h+l \leq$ $k$, for every $h$ tuples of points $p_{1}, \ldots, p_{h} \in M_{4 \delta}$ and $B_{p_{i}}(2 r) \subset M_{2 \delta}$ and for every $l$ tuples of points $q_{1}, \ldots, q_{l} \in \partial M$ we have

$$
\int_{M_{4 \delta} \cap\left(\cup_{i=1}^{h} B_{p_{i, u}}(r)\right)} e^{4 u} d V_{g}<\int_{M_{4 \delta}} f d V_{g}-\epsilon
$$

and

$$
\int_{\partial M \times\left[0, \delta / 4 \cap\left(\cup_{i=1}^{l} B_{q_{i, u}}^{+}(r)\right)\right.} e^{4 u} d V_{g}<\int_{\partial M \times[0, \delta / 4]} f d V_{g}-\epsilon .
$$

Now since the arguments we will carry out work for all the three cases, then we will focus only on case 3 . We assume that this is the case and we apply Lemma 4.2 with $f=e^{4 u_{n}}$, and in turn Lemma 4.1 with $\delta_{0}=2 \bar{r}, S_{i}=B_{\bar{p}_{i}}(\bar{r})$, $\Omega_{j}=B_{\bar{q}_{j}}^{+}(\bar{r})$ and $\gamma_{0}=\bar{\epsilon}$, where $\bar{\epsilon}, \bar{r}, \bar{p}_{i}$ and $\bar{q}_{i}$ are given as in Lemma 4.2. 
Thus we have for every $\tilde{\epsilon}>0$ there exists $C$ depending on $\epsilon, r, \delta$ and $\tilde{\epsilon}$ such that

$$
\begin{aligned}
\mathrm{II}\left(u_{n}\right) \geq & \left\langle P_{g}^{4,3} u_{n}, u_{n}\right\rangle+4 \int_{M} Q_{g} u_{n} d V_{g}+4 \int_{\partial M} T_{g} u_{n} d S_{g} \\
& -\frac{\kappa_{P^{4}, P^{3}}}{4 \pi^{2}(2 \tilde{h}+\tilde{l}-\tilde{\epsilon})}\left\langle P_{g}^{4,3} u_{n}, u_{n}\right\rangle-C \kappa_{P^{4}, P^{3}}-4 \kappa_{P^{4}, P^{3}} \overline{u_{n}},
\end{aligned}
$$

where $\tilde{h}$ and $\tilde{l}$ are given as in Lemma 4.2 and $C$ is independent of $n$. On the other hand, using the fact that $2 \tilde{h}+\tilde{l} \geq k+1$, we have that

$$
\begin{aligned}
\mathrm{II}\left(u_{n}\right) \geq & \left\langle P_{g}^{4,3} u_{n}, u_{n}\right\rangle+4 \int_{M} Q_{g} u_{n} d V_{g}+4 \int_{\partial M} T_{g} u_{n} d S_{g} \\
& -\frac{\kappa_{P^{4}, P^{3}}}{4 \pi^{2}(k+1-\tilde{\epsilon})}\left\langle P_{g}^{4,3} u_{n}, u_{n}\right\rangle-C \kappa_{P^{4}, P^{3}}-4 \kappa_{P^{4}, P^{3}} \overline{u_{n}} .
\end{aligned}
$$

So, since $\kappa_{P^{4}, P^{3}}<(k+1) 4 \pi^{2}$, by choosing $\tilde{\epsilon}$ small we get

$$
\mathrm{II}\left(u_{n}\right) \geq \beta\left\langle P_{g}^{4,3} u_{n}, u_{n}\right\rangle-4 C\left\langle P_{g}^{4,3} u_{n}, u_{n}\right\rangle^{1 / 2}-C \kappa_{P^{4}, P^{3}},
$$

thanks to the Hölder inequality, Sobolev embedding, trace Sobolev embedding and to the fact that Ker $P_{g_{0}}^{4,3} \simeq \mathbb{R}$ (where $\left.\beta=1-\frac{\kappa_{P^{4}, P^{3}}}{4 \pi^{2}(k+1-\tilde{\epsilon})}>0\right)$. Thus we arrive to

$$
\mathrm{II}\left(u_{n}\right) \geq-C .
$$

So we reach a contradiction. Hence the lemma is proved.

Next we give a lemma which is a direct consequence of the previous one. It gives the distance of the functions $e^{4 u}$ (suitably normalized) from $\left(M_{\partial}\right)_{k}$.

Corollary 4.4. Let $\bar{\varepsilon}$ be a (small) arbitrary positive number and $k$ be given as in (1.6). Then there exists $L>0$ such that if $\operatorname{II}(u) \leq-L$ and $\int_{\Sigma} e^{4 u} d V_{g}=$ 1 , then we have that $d_{M}\left(e^{4 u},\left(M_{\partial}\right)_{k}\right) \leq \bar{\varepsilon}$.

Proof. Let $\epsilon>0, r>0$ (to be fixed later) and let $L$ be the corresponding constant given by Lemma 4.3. Now let $\delta>0$, then by Lemma 4.3 we have the following three situations:

a) Conclusion 1 in Lemma 4.3 holds;

b) Conclusion 2 in Lemma 4.3 holds; or

c) Conclusion 3 in Lemma 4.3 holds. 
Suppose that a) holds. Since the same arguments can be carried out for the other cases, then we will only consider this case. We have that by Lemma 4.3 , there exists $\tilde{k}$ points $p_{1}, \ldots, p_{\tilde{k}}$ verifying (4.17). Next we define $\sigma \in\left(M_{\partial}\right)_{k}$ as follows:

$$
\begin{gathered}
\sigma=\sum_{i=1}^{\tilde{k}} t_{i} \delta_{p_{i}} \text { where } t_{i}=\int_{A_{r, i}} e^{4 u} d V_{j}, \\
A_{r, i}:=B_{p_{i}}(r) \backslash \cup_{s=1}^{i-1} B_{p_{s}}(r), \quad i=1, \ldots, \tilde{k}-1, \quad t_{\tilde{k}}=1-\sum_{i=1}^{\tilde{k}-1} t_{i} .
\end{gathered}
$$

By construction we have $A_{r, i}$ are disjoint and $\cup_{i=1}^{\tilde{k}-1} A_{r, i}=\cup_{i=1}^{\tilde{k}-1} B_{p_{i}}(r)$. Now let $\varphi \in C^{1}(M)$ be such that $\|\varphi\|_{C^{1}(M)} \leq 1$. By triangle inequality, the mean value theorem and the integral estimate in Lemma 4.3, we have that the following estimate holds

$$
\left|\int_{M} e^{4 u} \varphi-<\sigma, \varphi>\right| \leq C_{M}\left(r+\epsilon+\int_{M_{2 \delta} \backslash M_{4 \delta}} e^{4 u} d V_{g}\right)
$$

where $C_{M}$ is a constant depending only on $M$. So, letting $\delta$ tend to zero and choosing $\epsilon$ and $r$ so small that $C_{M}(r+\epsilon)<\frac{\bar{\epsilon}}{2}$, we obtain

$$
d_{M}\left(e^{4 u},\left(M_{\partial}\right)_{k}\right)<\bar{\epsilon}
$$

as desired.

\subsection{Mapping sublevels of II into $\left(M_{\partial}\right)_{k}$}

In this subsection, we show that $\left(M_{\partial}\right)_{k}$ is not contractible and prove the existence of a non-trivial projection from some sublevels of the functional II into the set $\left(M_{\partial}\right)_{k}$. Since the Euler-Lagrange functional II is invariant under translation by a constant, we can always assume that the functions in $u \in H_{\partial / \partial n}$ we are dealing with satisfies the normalization $\int_{M} e^{4 u} d V_{g}=1$.

As already said in the introduction of the subsection, we begin with the following lemma.

Proposition 4.5. For any $k \geq 1$, the set $\left(M_{\partial}\right)_{k}$ is not contractible.

Before making the proof of the proposition, we recall the following wellknown result, whose proof can be found in [14]. 
Lemma 4.6. For any $k \geq 1$, the set $(\partial M)_{k}$ is not contractible, indeed $H_{4 k-1}\left((\partial M)_{k} ; \mathbb{Z}_{2}\right) \neq 0$.

The next lemma is a trivial consequence of normal geodesics at the boundary.

Lemma 4.7. There exists a small $\epsilon_{0}>0$ such that a continuous projection

$$
P_{\partial M}:(\partial M)^{\epsilon_{0}} \longrightarrow \partial M
$$

exists.

Proof of Proposition 4.5. Suppose that the following claim is true, $(\partial M)_{k}$ is a deformation retract of some of its open neighborhood $U$ in $\left(M_{\partial}\right)_{k}$ such that setting $V=\left(M_{\partial}\right)_{k} \backslash(\partial M)_{k}$, we have that $X=U \cup \operatorname{int}(V) \simeq\left(M_{\partial}\right)_{k}$. Now assuming that the claim holds, we have that

$$
H_{4 k-1}\left(X ; \mathbb{Z}_{2}\right) \simeq H_{4 k-1}\left(\left(M_{\partial}\right)_{k} ; \mathbb{Z}_{2}\right)
$$

and

$$
H_{4 k-1}\left(U ; \mathbb{Z}_{2}\right) \simeq H_{4 k-1}\left((\partial M)_{k} ; \mathbb{Z}_{2}\right)
$$

Next let us denote

$$
i: U \cap V \rightarrow U, \quad j: U \cap V \rightarrow V, \quad m: U \rightarrow X, \quad t: V \rightarrow X
$$

the canonical injections and by $i_{*}, j_{*}, m_{*}, t_{*}$ the corresponding homomorphism on homology groups.

We have that by the Mayers-Vietoris theorem there exists a homomorphism $\Delta: H_{p}\left(\left(M_{\partial}\right)_{k}\right) \rightarrow H_{p-1}\left(\left(M_{\partial}\right)_{k}\right)$ (where $p$ is a generic positive integer number) such that the following sequence is exact.

$$
\begin{aligned}
\cdots & \stackrel{\Delta}{\rightarrow} H_{4 k-1}\left(U \cap V ; \mathbb{Z}_{2}\right) \stackrel{\left(i_{*}, j_{*}\right)}{\rightarrow} H_{4 k-1}\left(U ; \mathbb{Z}_{2}\right) \oplus H_{4 k-1}\left(V ; \mathbb{Z}_{2}\right) \\
& \stackrel{m_{*}-t_{*}}{\rightarrow} H_{4 k-1}\left(X ; \mathbb{Z}_{2}\right) \stackrel{\Delta}{\rightarrow} H_{4 k-2}\left(U \cap V ; \mathbb{Z}_{2}\right) \stackrel{\left(i_{*}, j_{*}\right)}{\rightarrow} \cdots
\end{aligned}
$$

Now for $h \in \mathbb{N}, l \in \mathbb{N}$ such that $h \leq \tilde{k}, l \leq k$ and $2 h+l \leq k$, we recall that $M_{h, l}$ (for the definition, see Section 2) is a stratified set, namely a union of sets of different dimensions. The maximal dimension is $5 h+4 l-1$, when 
all the points are distinct and the coefficients belong to $(0,1)$. Hence the following holds

$$
\operatorname{dim}\left(M_{h, l} \cap V\right) \leq 5 h+4 l-1
$$

and if $h=0$, then

$$
M_{0, l} \cap V=\emptyset
$$

Hence from the trivial identity $5 h+4 l-1<4 k-2$ for such a $(h, l)$ with $h \neq 0$, we infer that

$$
H_{4 k-1}\left(U \cap V ; \mathbb{Z}_{2}\right)=H_{4 k-1}\left(V ; \mathbb{Z}_{2}\right)=H_{4 k-2}\left(U \cap V ; \mathbb{Z}_{2}\right)=0
$$

Thus from (4.23) we deduce that

$$
H_{4 k-1}\left(U ; \mathbb{Z}_{2}\right) \simeq H_{4 k-1}\left(X ; \mathbb{Z}_{2}\right)
$$

So using Lemma 4.6, and formulas (4.21) and (4.22), we get

$$
H_{4 k-1}\left(\left(M_{\partial}\right)_{k} ; \mathbb{Z}_{2}\right) \neq 0
$$

Hence to complete the proof of the lemma, it is sufficient to prove the claim. Now let us make its proof.

First of all it is easy to see that there exist $\epsilon>0\left(4 \epsilon<\epsilon_{0}\right)$ small enough and a continuous map

$$
X_{\partial}:[0,1] \times(\partial M)^{2 \epsilon} \longrightarrow(\partial M)^{2 \epsilon}
$$

such that

$$
X_{\partial}(0, \cdot)=I_{(\partial M)^{2 \epsilon}}(\cdot), \quad X_{\partial}(1, \cdot)=P_{\partial M}(\cdot)
$$

where $P_{\partial}$ is given by Lemma 4.7 .

Next, we define a homotopy $F:[0,1] \times B_{2 \epsilon, k} \longrightarrow B_{2 \epsilon, k}$ (for the definition of $B_{2 \epsilon, k}$, see Section 2) whose construction is based on the following idea. Given $\sigma=\sigma_{\text {int }}+\sigma_{\text {bdry }} \in B_{2 \epsilon, k}, \sigma_{\text {int }}=\sum_{i=1}^{h} t_{i} \delta_{x_{i}}, \sigma_{\text {bdry }}=\sum_{i=1}^{l} s_{i} \delta_{y_{i}}$, $h \leq \tilde{k}, l \leq k, 2 h+l \leq k$, we fixed the boundary part, namely $\sigma_{\text {bdry }} \in B_{2 \epsilon, k}$. And for the interior part $\sigma_{\text {int }}$, we argue as follows: if $x_{i}$ is closed to the boundary at distance less than $\epsilon$, we send $t_{i} \delta_{x_{i}}$ to $t_{i} \delta_{P_{\partial M}\left(x_{i}\right)}$, and if it is far from the boundary, say at distance bigger than $2 \epsilon$, we squeeze and in 
the intermediate regime we use an homotopy argument reflecting the possibility between squeezing and projection to boundary via $P_{\partial M}$ since the distance is less than or equal to $2 \epsilon$. More precisely, we define the homotopy $F:[0,1] \times B_{2 \epsilon, k} \longrightarrow B_{2 \epsilon, k}$ as follows:

For every $\sigma=\sigma_{\text {int }}+\sigma_{\text {bdry }} \in B_{2 \epsilon, k} \quad$ with $\quad \sigma_{\text {int }}=\sum_{i=1}^{h} t_{i} \delta_{x_{i}}, \quad \sigma_{\text {bdry }}=$ $\sum_{i=1}^{l} s_{i} \delta_{y_{i}}$ and $s \in[0,1]$, we set

$$
F(\sigma, s)=\sigma(s)+\sigma_{\text {bdry }}
$$

where $\sigma(s)$ is defined as

$$
\sigma(s)=\sum_{i=1} t_{i}(s) \delta_{x_{i}(s)}
$$

and

$$
t_{i}(s) \delta_{x_{i}(s)}=\left\{\begin{array}{l}
\left(1-\frac{s}{2}\right) \gamma(s) t_{i} \delta_{X_{\partial}\left(s, x_{i}\right)} \text { if } \operatorname{dist}\left(x_{i}, \partial M\right) \leq \epsilon \\
\left(1-s \frac{\operatorname{dist}\left(x_{i}, \partial M\right)}{2 \epsilon}\right) \gamma(s) t_{i} \delta_{X_{\partial}\left(2-\operatorname{dist}\left(x_{i}, \partial M\right) / \epsilon, x_{i}\right)} \\
\quad \text { if } \epsilon \leq \operatorname{dist}\left(x_{i}, \partial M\right) \leq 2 \epsilon \\
(1-s) \gamma(s) t_{i} \delta_{x_{i}} \\
\quad \text { if } \operatorname{dist}\left(x_{i}, \partial M\right) \geq 2 \epsilon,
\end{array}\right.
$$

where $\gamma(s)$ is such that we have the normalization $\sum_{i=1}^{h} t_{i}(s)+\sum_{i=1}^{l} s_{i}=1$. Thus by trivial calculations, we obtain

$$
\gamma(s)=\frac{\sum_{i-=1}^{l} t_{i}}{\sum_{d\left(x_{i}, \partial M\right)<\epsilon}\left((1-(s / 2)) t_{i}\right)+\sum_{\epsilon \leq d\left(x_{i}, \partial M\right)<2 \epsilon}\left(\left(1-s\left(\operatorname{dist}\left(x_{i}, \partial M\right)\right.\right.\right.} .
$$

So by setting $U=B_{2 \epsilon, k}$, we have that the claim is proved. Hence the proof of the proposition is complete.

Next we give a proposition which asserts the existence of a non-trivial continuous global projection $\Psi$ from some negative sublevels of II onto $\left(M_{\partial}\right)_{k}$. By non-triviality of $\Psi$, we mean that the induced map $\Psi_{*}$ on homology group is not zero. This fact follows directly from the non-contractibility of $\left(M_{\partial}\right)_{k}$ and b of Proposition 4.14 .

Proposition 4.8. For $k \geq 1$ given as in (1.6), there exists a large $L>$ 0 and a continuous map $\Psi$ from the sublevel $\left\{\mathrm{II}(u)<-L, \int_{M} e^{4 u} d V_{g}=1\right\}$ onto $\left(M_{\partial}\right)_{k}$ which is topologically non-trivial. 
We start by giving an auxillary lemma which will be used in the proof of the Proposition. The lemma states (roughly) that $M$ can be embedded smoothly in Euclidean space (with large dimension) such that its interior lies in the interior of the positive half space and its boundary at the one (boundary) of that half space. Since the proof works for all dimensions, we will give the lemma for a general finite-dimensional compact smooth manifold with smooth boundary. Precisely, we have

Lemma 4.9. Suppose $N$ is a smooth $n$-dimensional compact manifold with smooth boundary. Then there exists $m \in \mathbb{N}^{*}$ (large enough) and $T: N \rightarrow$ $\mathbb{R}^{m+1}$ an embedding such that, $T(\partial N) \subset \partial \mathbb{R}_{+}^{m+1}, T(\operatorname{int}(N)) \subset \operatorname{int}\left(\mathbb{R}_{+}^{m+1}\right)$, and $T: \operatorname{int}(N) \rightarrow \operatorname{int}\left(\mathbb{R}_{+}^{m+1}\right)$ is smooth. Furthermore, there holds for all $x \in \partial N$, the vector $\nu_{x}$ with origin $T(x)$ and parallel to the $x_{m+1}$-axis is the normal vector of $T(\partial N)$ at $T(x)$.

Proof. First of all, by Whitney's embedding theorem we have that there exists $m \in \mathbb{N}^{*}$ such that $N$ is smoothly embedded in $\mathbb{R}^{m}$, namely there exists $\hat{T}: N \rightarrow \mathbb{R}^{m}$ a smooth embedding. Now, we extend $N$ by adding a nice tubular neighborhood such that the resulting object is a compact smooth manifold that we denote by $\hat{N}$. Using the compactness of $\hat{N}$, we can find a finite open covering $\left\{\Theta_{i}\right\}_{1=1}^{k}$ of $N$ and a finite number of smooth functions $\varphi_{i}: \Theta_{i} \rightarrow \mathbb{R}^{n}$ such that $\left\{\left(\Theta_{i}, \varphi_{i}\right)\right\}_{1}^{k}$ are local coordinates for $N$ and $\Theta_{i} \subset \hat{N}$. Moreover, we can take $\Theta_{i}$ such that if $\Theta_{i} \cap \partial N \neq \emptyset$, then the associated $\varphi_{i}$ verifies the following properties:

$$
\begin{aligned}
\varphi_{i}: \Theta_{i} & \rightarrow[-1,1]^{n}, \\
\varphi_{i}: \Theta_{i} \cap N & \rightarrow[-1,1]^{n} \cap\left\{x_{n}>0\right\}, \\
\varphi_{i}: \Theta_{i} \cap \partial N & \rightarrow[-1,1]^{n} \cap\left\{x_{n}=0\right\},
\end{aligned}
$$

and furthermore $\varphi_{i}$ maps the outward normal vectors on $\partial N$ to the outward normal vectors at $\partial \mathbb{R}_{+}^{n}$ of $[-1,1]^{n} \cap\left\{x_{n}=0\right\}$.

Now to the covering $\left\{\Theta_{i}\right\}_{i=1}^{k}$, we associate a finite number of functions $\left\{h_{i}\right\}_{1=1}^{k} h_{i}: \Theta_{i} \rightarrow \mathbb{R}$ as follows:

$$
h_{i}(x)= \begin{cases}1, & x \in \Theta_{i} \subset \operatorname{int}(N), \\ h \circ \varphi_{i}(x), & x \in \Theta_{i} \cap \partial N,\end{cases}
$$


where $h:[-1,1]^{n} \rightarrow \mathbb{R}$ is defined as follows

$$
h(x)= \begin{cases}0 & \text { if } x \in[-1,1]^{n} \cap\left\{x_{n}<0\right\}, \\ \sqrt{1-\left(x_{n}-1\right)^{2}} & \text { if } x \in[-1,1]^{n} \cap\left\{x_{n} \geq 0\right\} .\end{cases}
$$

Next we choose a partition of unity $\left\{g_{i}\right\}_{1}^{k}$ subordinated to the covering $\left\{\Theta_{i}\right\}_{1}^{k}$. Therefore, $g_{i}$ satifies

$$
\begin{cases}g_{i} \in C_{c}^{\infty}\left(\Theta_{i}\right), & 1 \leq i \leq k \\ 0 \leq g_{i} \leq 1 & \text { on } N \forall i \\ \sum_{i=1}^{k} g_{i}=1 & \text { on } N\end{cases}
$$

With this partition of unity and the functions $h_{i}$, we set

$$
u(x)=\sum_{i=1}^{k} g_{i}(x) h_{i}(x) .
$$

Using the definition of $h_{i}$, one can check easily that $u$ verifies the following properties:

$$
\begin{gathered}
u(x)>0 \forall x \in \operatorname{int}(N) \text { and } u \in C^{\infty}(\operatorname{int}(N)), \\
u(x)=0 \forall x \in \partial N \quad \text { and } \quad \frac{\partial u}{\partial n}=+\infty \text { on } \partial N,
\end{gathered}
$$

where $\frac{\partial}{\partial n}$ stands for the inward normal derivative at $\partial N$.

Now for $x \in N$ we define $T: N \rightarrow \mathbb{R}^{m+1}$ as follows:

$$
T(x)=(\hat{T}(x), u(x))
$$

where $\hat{T}$ is given by the Whitney embedding theorem.

It is obvious that $T$ is an embedding, smooth $\operatorname{in} \operatorname{int}(N)$ and satisfies the properties of the lemma. Hence the proof is completed.

Next we will use the previous lemma to define a special doubling of $M$ such that it is $C^{1}$. First of all applying $M$ we get the existence of an embedding $T: M \rightarrow \mathbb{R}^{m+1}$ (given by Lemma 4.9 ).

Now we define the reflection $\tilde{T}$ of $T$ as follows:

$$
\tilde{T}(x)=\left(T^{1}(x), \ldots, T^{m}(x),-T^{m+1}(x)\right),
$$

where $T(x)=\left(T^{1}(x), \ldots, T^{m}(x), T^{m+1}(x)\right)$. From the properties of $T$, it is easily seen that $\tilde{T}$ is also an embedding of $M$. 
With the embeddings $T$ and $\tilde{T}$, we can define the desired doubling of $M$. To do so, we start by making some notations. We set

$$
D M^{+}=T(M) \quad \text { and } \quad D M^{-}=\tilde{T}(M) .
$$

By the properties of $T$ and $\tilde{T}$ (see Lemma 4.9) we have that $D M^{+}$and $D M^{-}$have a common boundary, which is $\partial M$. Moreover, they have the same normal vectors at their common boundary. Now we are ready to define the doubling of $M$ and denote it by $D M$ as follows:

$$
D M=D M^{+\cup D} M^{-},
$$

where the notation $\sim$ means we identify $T(x)$ and $\tilde{T}(x)$ for $x \in \partial M$.

Using the fact that $D M^{+}$and $D M^{-}$have the same normal at $\partial M$ and by considering the reflection $\bar{g}$ of $g$ through $\partial M$, we derive that $(D M, \bar{g})$ is a $C^{1}$-closed 4-dimensional Riemannian manifold with the Lipschitz metric.

Next we introduce some further definitions.

Given a point $x=\left(x_{1}, \ldots, x_{m+1}\right) \in D M$, we define the even reflection of $x$ across $\partial M$ and denote it by $\hat{x}$ as follows:

$$
\hat{x}=\left(x_{1}, \ldots, x_{m},-x_{m+1}\right) .
$$

For a function $u \in H^{2}(M)$ and identifying $D M^{+}$to $M$, we define the even reflection of $u$ across $\partial M$ as follows:

$$
u_{D M}(x)= \begin{cases}u(x) & \text { if } x \in D M^{+} \\ u(\hat{x}) & \text { if } x \in D M^{-}\end{cases}
$$

We say that a function $u \in L^{1}(D M)$ is even with respect to the boundary $\partial M$ if

$$
u(x)=u(\hat{x}) \text { for a.e } \quad x \in D M .
$$

We denote by $D M_{k}$ the $k$ barycenters relative to $D M$ of order $k$, namely

$$
D M_{k}=\left\{\sum_{i=1}^{k} t_{i} \delta_{x_{i}}, x_{i} \in D M, \sum_{i=1}^{k} t_{i}=1\right\} .
$$

We have that $D M_{k}$ is a stratified set, namely a union of sets of different dimensions, with maximal dimension being $5 k-1$ (for more information, see [14]). It will be endowed with the weak topology of distributions. To prove 
Proposition 4.8, we will need at one stage to (roughly speaking) evaluate the distance of some suitable functions to $D M_{k}$ (see formula (4.27) below). To do this, we will adopt the metric distance given by $C^{1}(D M)^{*}$ and inducing the same topology as the weak topology of distributions and will be denoted by $d_{D M}(\cdot, \cdot)$.

For $\epsilon>0$, we set

$$
D_{\epsilon, k, D M}=\left\{f \in L^{1}(D M), \quad f \geq 0, \quad \int_{D M} f d V_{\bar{g}}=1 \text { and } d_{D M}\left(f, D M_{k}\right) \leq \epsilon\right\}
$$

The next discussion concerns the way of defining convex combination of points of $D M$ belonging to a small metric ball. To do so, we use the embedding of $D M$ in $\mathbb{R}^{m+1}$ discussed above in the following way. Given points $x_{i} \in D M, i=1, \ldots, l$, which belong to a small metric ball and $\alpha_{i} \geq 0$, $i=1, \ldots, l, \sum_{i=1}^{l} \alpha_{i}=1$, we define their convex combination denoted by $\sum_{i=1}^{l} \alpha_{i} x_{i}$ by considering the convex combination of their image under the embedding and after projecting the result on the image of $D M$ (which is also identified to $D M$ ). Hence in this way we have that for such a type of points, the convex combination is well defined and if $d\left(x_{i}, x_{j}\right) \leq \beta$, then we obtain $d\left(x_{i}, \sum_{j=1}^{l} \alpha_{j} x_{j}\right) \leq 2 \beta$.

We recall that the arguments which have led to Proposition 3.1 in [14] are based on the construction of some partial projections on some suitable subsets $M_{j}\left(\epsilon_{j}\right)$ (obtained by removing singularities) of $M_{k}$ and gluing method based on the construction of a suitable homotopy. The construction of the latter homotopy which is not trivial is based on some weighted convex combinations and the fact that the underlying manifold does not have corners.

Using the notion of convex combinations discussed above and the fact that $D M$ is a $C^{1}$-closed Riemannian manifold with Lipschitz metric which rule out the presence of corners, and an adaptation of the arguments of Proposition 3.1 in [14], we have the following lemma:

Lemma 4.10. Let $k \geq 1$ be as in (1.6) and $D M$ be as above. Then there exists $\epsilon_{k, D M}$ such that for every $\epsilon \leq \epsilon_{k, D M}$, we have the existence of a nontrivial continuous projection

$$
P_{\epsilon, k, D M}: D_{\epsilon, k, D M} \rightarrow D M_{k}
$$

with the following property: 
for every $u \in D_{\epsilon, k, D M}$ even (in the sense of (4.25)) if $P_{\epsilon, k, D M}(u)=\sum_{i=1}^{k} t_{i} \delta_{x_{i}}$, then

$$
\forall x_{i} \notin \partial M \text {; there exists } j \neq i \quad \text { such that } \quad x_{j}=\hat{x}_{i} \text { and } t_{j}=t_{i} \text {. }
$$

Now we are ready to make the proof of the proposition.

Proof of Proposition 4.8. To begin, we let $\epsilon_{k}$ be so small that Lemma 4.10 holds with $\epsilon=\epsilon_{k}$. Next applying Corollary 4.4 with $\bar{\epsilon}=\frac{\epsilon_{k}}{4}$, we obtain the existence of $L$ (large enough) such that

$$
\forall u \in H_{\partial / \partial n}, \quad \int_{M} e^{4 u} d V_{g}=1 \quad \text { and } \quad \mathrm{II}(u) \leq-L
$$

there holds

$$
4 d\left(e^{4 u},\left(M_{\partial}\right)_{k}\right) \leq \epsilon_{k} .
$$

Now since for $u \in H_{\partial / \partial n}$, we have by definition of $H_{\partial / \partial n}$ that

$$
\frac{\partial u}{\partial n_{g}}=0
$$

then we infer that the even reflection $u_{D M}$ of $u$ belongs to $H^{2}(D M)$. Moreover, we have also that the map

$$
u \in H^{2}(M) \rightarrow u_{D M} \in H^{2}(D M) \text { is continuous. }
$$

On the other hand, one can easily check (using the evenness of $\bar{g}$ ) that the following distance estimate holds:

$$
d_{D M}\left(\frac{e^{4 u_{D M}}}{\int_{D M} e^{4 u_{D M}} d V_{\bar{g}}}, D M_{k}\right)<\epsilon_{k}
$$

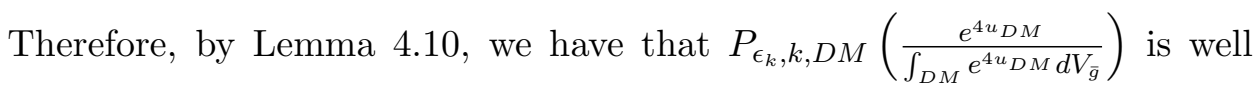
defined and belongs to $D M_{k}$. Moreover, still from Lemma 4.10 we have 
that if

$$
P_{\epsilon_{k}, k, D M}\left(\frac{e^{4 u_{D M}}}{\int_{D M} e^{4 u_{D M}} d V_{\bar{g}}}\right)=\sum_{i=1}^{k} t_{i} \delta_{x_{i}}
$$

then the following holds:

$\forall x_{i} \notin \partial M$ there exists $j \neq i \quad$ such that $\quad x_{j}=\hat{x}_{i}$ and $t_{j}=t_{i}$.

Thus setting

$$
\Psi(u)=\frac{1}{\sum_{x_{a} \in \operatorname{int}\left(D M^{+}\right)} t_{a}+\sum_{x_{b} \in \partial M} t_{b}}\left(\sum_{x_{i} \in \operatorname{int}\left(D M^{+}\right)} t_{i} \delta_{x_{i}}+\sum_{x_{j} \in \partial M} t_{j} \delta_{x_{j}}\right),
$$

we get $\Psi(u) \in\left(M_{\partial}\right)_{k}$. On the other hand, since the map $v \in H^{2}(D M) \rightarrow$ $e^{4 v} \in L^{1}(D M)$ is continuous, then from (4.26) we derive that the map $u \in$ $H_{\partial / \partial \eta} \rightarrow e^{4 u_{D M}} \in L^{1}(D M)$ is continuous, too. Thus from the continuity of $P_{\epsilon_{k}, k, D M}$ we infer that $\Psi$ is also continuous. Hence the proof of the proposition is complete.

\subsection{Mapping $\left(M_{\partial}\right)_{k}$ into sublevels of II}

In this subsection we will define some test functions depending on a real parameter $\lambda$ and give estimate of the quadratic part of the functional II on those functions as $\lambda$ tends to infinity. And as a corollary we define a continuous map from $\left(M_{\partial}\right)_{k}$ into large negative sublevels of II.

For $\delta>0$ small, consider a smooth non-decreasing cut-off function $\chi_{\delta}$ : $\mathbb{R}_{+} \rightarrow \mathbb{R}$ satisfying the following properties (see [14]):

$$
\begin{cases}\chi_{\delta}(t)=t, & \text { for } t \in[0, \delta] \\ \chi_{\delta}(t)=2 \delta, & \text { for } t \geq 2 \delta, \\ \chi_{\delta}(t) \in[\delta, 2 \delta], & \text { for } t \in[\delta, 2 \delta]\end{cases}
$$

Then, given $\sigma=\sigma_{\text {int }}+\sigma_{\text {bdry }} \in\left(M_{\partial}\right)_{k}, \sigma_{\text {int }}=\sum_{i=1}^{h} t_{i} \delta_{x_{i}}, \sigma_{\text {bdry }}=\sum_{i=1}^{l} s_{i} \delta_{q_{i}}$ and $\lambda>0$, we define the function $\varphi_{\lambda, \sigma \text {,int }}: M \rightarrow \mathbb{R}, \varphi_{\lambda, \sigma, \text { bdry }}: M \rightarrow \mathbb{R}$ and 
$\varphi_{\lambda, \sigma}: M \rightarrow \mathbb{R}$ as follows:

$$
\begin{gathered}
\varphi_{\lambda, \sigma, \text { int }}(y)=\frac{1}{4} \log \left[\sum_{i=1}^{h} t_{i}\left(\frac{2 \lambda}{1+\lambda^{2} \chi_{\delta}^{2}\left(d_{1, i}(y)\right)}\right)^{4}\right], \\
\varphi_{\lambda, \sigma, \operatorname{bdry}}(y)=\frac{1}{4} \log \left[\sum_{i=1}^{l} s_{i}\left(\frac{2 \lambda}{1+\lambda^{2} \chi_{\delta}^{2}\left(d_{2, i}(y)\right)}\right)^{4}\right]
\end{gathered}
$$

and

$$
\varphi_{\lambda, \sigma}=\varphi_{\lambda, \sigma, \mathrm{int}}+\varphi_{\lambda, \sigma, \mathrm{bdry}}
$$

where we have set

$$
\begin{gathered}
d_{1, i}(y)=\operatorname{dist}\left(y, x_{i}\right), \quad x_{i} \in \operatorname{int}(M), \quad y \in M, \\
d_{2, i}(y)=\operatorname{dist}\left(y, q_{i}\right), \quad q_{i} \in \partial M, \quad y \in M,
\end{gathered}
$$

with $\operatorname{dist}(\cdot, \cdot)$ denoting the Riemannian distance on $M$.

Now we state a lemma giving an estimate (uniform in $\sigma \in\left(M_{\partial}\right)_{k}$ ) of the quadratic part $\left\langle P_{g}^{4,3} \varphi_{\lambda, \sigma}, \varphi_{\lambda, \sigma}\right\rangle$ of the Euler functional II as $\lambda \rightarrow+\infty$. Its proof is a straightforward adaptation of the arguments in Lemma 4.5 in [18] with the dimension being 4 .

Lemma 4.11. Suppose $\varphi_{\lambda, \sigma}$ as in (4.28) and let $\epsilon>0$ be small enough. Then as $\lambda \rightarrow+\infty$, one has

$$
\left\langle P_{g}^{4,3} \varphi_{\lambda, \sigma}, \varphi_{\lambda, \sigma}\right\rangle \leq\left(16 \pi^{2} k+\epsilon+o_{\delta}(1)\right) \log \lambda+C_{\epsilon, \delta} .
$$

Next we state a lemma giving estimates of the remainder part of the functional II along $\varphi_{\sigma, \lambda}$. The proof is the same as the one of formulas (3.10) and (3.11) in the proof of Lemma 4.3 in [14].

Lemma 4.12. Suppose $\varphi_{\sigma, \lambda}$ as in (4.28). Then as $\lambda \rightarrow+\infty$, one has

$$
\begin{aligned}
& \int_{M} Q_{g} \varphi_{\sigma, \lambda} d V_{g}=-\kappa_{P_{g}^{4}} \log \lambda+O\left(\delta^{4} \log \lambda\right)+O(\log \delta)+O(1), \\
& \int_{\partial M} T_{g} \varphi_{\sigma, \lambda} d V_{g}=-\kappa_{P_{g}^{3}} \log \lambda+O\left(\delta^{3} \log \lambda\right)+O(\log \delta)+O(1)
\end{aligned}
$$

and

$$
\log \int_{M} e^{4 \varphi_{\sigma, \lambda}}=O(1)
$$


Now for $\lambda>0$ we define the map $\Phi_{\lambda}:\left(M_{\partial}\right)_{k} \rightarrow H_{\partial / \partial n}$ by the following formula:

$$
\forall \sigma \in M_{k} \Phi_{\lambda}(\sigma)=\varphi_{\sigma, \lambda}
$$

We have the following lemma which is a trivial application of Lemmas 4.11 and 4.12 .

Lemma 4.13. For $k \geq 1$ (given as in (1.6)), given any $L>0$ large enough, there exist a small $\delta$ and a large $\bar{\lambda}$ such that $\mathrm{II}\left(\Phi_{\bar{\lambda}}(\sigma)\right) \leq-L$ for every $\sigma \in$ $\left(M_{\partial}\right)_{k}$.

Next we state a proposition giving the existence of the projection from $\left(M_{\partial}\right)_{k}$ into large negative sublevels of II, and the non-triviality of the map $\Psi$ of Proposition 4.8 .

Proposition 4.14. Let $\Psi$ be the map defined in Proposition 4.8. Then assuming $k \geq 1$ (given as in (1.6)), for every $L>0$ sufficiently large (such that Proposition 4.8 applies), there exists a map

$$
\Phi_{\bar{\lambda}}:\left(M_{\partial}\right)_{k} \longrightarrow H_{\partial / \partial n}
$$

with the following properties:

a)

$$
\operatorname{II}\left(\Phi_{\bar{\lambda}}(z)\right) \leq-L \text { for any } z \in\left(M_{\partial}\right)_{k}
$$

b) $\Psi \circ \Phi_{\bar{\lambda}}$ is homotopic to the identity on $\left(M_{\partial}\right)_{k}$.

Proof. The statement (a) follows from Lemma 4.13. To prove (b), it is sufficient to consider the family of maps $T_{\lambda}:\left(M_{\partial}\right)_{k} \rightarrow\left(M_{\partial}\right)_{k}$ defined by

$$
T_{\lambda}(\sigma)=\Psi\left(\Phi_{\lambda}(\sigma)\right), \quad \sigma \in M_{k}
$$

We recall that when $\lambda$ is sufficiently large, then this composition is well defined. Therefore, since $\frac{e^{4 \varphi_{\sigma}, \lambda}}{\int_{M} e^{4 \varphi_{\sigma, \lambda}} d V_{g}} \rightarrow \sigma$ in the weak sense of distributions, letting $\lambda \rightarrow+\infty$ we obtain an homotopy between $\Psi \circ \Phi$ and $\operatorname{Id}_{\left(M_{\partial}\right)_{k}}$. This concludes the proof.

\subsection{Min-max scheme}

In this subsection, we describe the min-max scheme based on the set $\left(M_{\partial}\right)_{k}$ in order to prove Theorem 1.1. As anticipated in the introduction, we define 
a modified functional $\mathrm{II}_{\rho}$ for which we can prove existence of solutions in a dense set of the values of $\rho$. Following an idea of Struwe (see [20]), this is done by proving the a.e. differentiability of the map $\rho \rightarrow \overline{\mathrm{II}}_{\rho}$ (where $\overline{\mathrm{II}}_{\rho}$ is the minimax value for the functional $\mathrm{II}_{\rho}$ ).

We now introduce the minimax scheme which provides existence of solutions for (1.8). Let $\widehat{\left(M_{\partial}\right)_{k}}$ denote the (contractible) cone over $\left(M_{\partial}\right)_{k}$, which can be represented as $\widehat{\left(M_{\partial}\right)_{k}}=\left(\left(M_{\partial}\right)_{k} \times[0,1]\right)$ with $\left(M_{\partial}\right)_{k} \times 0$ collapsed to a single point. First let $L$ be so large that Proposition 4.8 applies with $\frac{L}{4}$, and then let $\bar{\lambda}$ be so large that Proposition 4.14 applies for this value of $L$. Fixing $\bar{\lambda}$, we define the following class:

(4.30) $\mathrm{II}_{\bar{\lambda}}=\left\{\pi: \widehat{\left(M_{\partial}\right)_{k}} \rightarrow H_{\partial / \partial n}: \pi\right.$ is continuous and $\left.\pi(\cdot \times 1)=\Phi_{\bar{\lambda}}(\cdot)\right\}$.

We then have the following properties.

Lemma 4.15. The set $\mathrm{II}_{\bar{\lambda}}$ is non-empty, and moreover, letting

$$
\overline{\mathrm{II}}_{\bar{\lambda}}=\inf _{\pi \in \mathrm{II}_{\bar{\lambda}}} \sup _{m \in \widehat{\left(M_{\partial}\right)_{k}}} \mathrm{II}(\pi(m)) \text {, there holds } \overline{\mathrm{II}}_{\bar{\lambda}}>-\frac{L}{2} .
$$

Proof. The proof is the same as the one of Lemma 5.1 in [14]. But we will repeat it for the reader's convenience.

To prove that $\overline{\mathrm{II}}_{\bar{\lambda}}$ is non-empty, we just notice that the following map

$$
\bar{\pi}(\cdot, t)=t \Phi_{\bar{\lambda}}(\cdot)
$$

belongs to $\mathrm{II}_{\bar{\lambda}}$. Now to prove that $\overline{\mathrm{II}}_{\bar{\lambda}}>-\frac{L}{2}$, let us argue by contradiction. Suppose that $\overline{\mathrm{II}}_{\bar{\lambda}} \leq-\frac{L}{2}$ : then there exists a map $\pi \in \mathrm{II}_{\bar{\lambda}}$ such that $\sup _{m \in \widehat{\left(M_{\partial}\right)_{k}}} \mathrm{II}(\pi(m)) \leq-\frac{3}{8} L$. Hence since Proposition 4.8 applies with $\frac{L}{4}$, writing $m=(z, t)$ with $z \in\left(M_{\partial}\right)_{k}$ we have that the map

$$
t \rightarrow \Psi \circ \pi(\cdot, t)
$$

is an homotopy in $\left(M_{\partial}\right)_{k}$ between $\Psi \circ \Phi_{\bar{\lambda}}$ and a constant map. But this is impossible since $\left(M_{\partial}\right)_{k}$ is non-contractible and $\Psi \circ \Phi_{\bar{\lambda}}$ is homotopic to the identity by Proposition 4.14.

Next we introduce a variant of the above minimax scheme, following $[14,18,20]$. For $\rho$ in a small neighborhood of $1,\left[1-\rho_{0}, 1+\rho_{0}\right]$, we define 
the modified functional $I I_{\rho}: H_{\partial / \partial n} \rightarrow \mathbb{R}:$

$$
\begin{aligned}
\mathrm{II}_{\rho}(u)= & \left\langle P_{g}^{4,3} u, u\right\rangle+4 \rho \int_{M} Q_{g} u d V_{g}+4 \rho \int_{\partial M} T_{g} u d S_{g} \\
& -4 \rho \kappa_{\left(P^{4}, P^{3}\right)} \log \int_{M} e^{4 u} d V_{g}, \quad u \in H_{\partial / \partial n} .
\end{aligned}
$$

Following the estimates of the previous section, one easily checks that the above minimax scheme applies uniformly for $\rho \in\left[1-\rho_{0}, 1+\rho_{0}\right]$ and for $\bar{\lambda}$ sufficiently large. More precisely, given any large number $L>0$, there exist $\bar{\lambda}$ sufficiently large and $\rho_{0}$ sufficiently small such that

$$
\begin{aligned}
& \sup _{\pi \in \mathrm{II}_{\bar{\lambda}}} \sup _{m \in \partial} \overline{\left(M_{\partial}\right)_{k}} \operatorname{II}(\pi(m))<-2 L, \\
& \overline{\mathrm{II}}_{\rho} \inf _{\pi \in \mathrm{II}_{\bar{\lambda}}} \sup _{m \in \overline{\left(M_{\partial}\right)_{k}}} \mathrm{II}_{\rho}(\pi(m))>-\frac{L}{2}, \quad \rho \in\left[1-\rho_{0}, 1+\rho_{0}\right],
\end{aligned}
$$

where $\mathrm{II}_{\bar{\lambda}}$ is defined as in (4.30). Moreover, using for example the test map, one shows that for $\rho_{0}$ sufficiently small there exists a large constant $\bar{L}$ such that

$$
\overline{\mathrm{II}}_{\rho} \leq \bar{L}, \quad \text { for every } \rho \in\left[1-\rho_{0}, 1+\rho_{0}\right]
$$

We have the following result regarding the dependence in $\rho$ of the minimax value $\overline{\mathrm{II}}_{\rho}$.

Lemma 4.16. Let $\bar{\lambda}$ and $\rho_{0}$ such that (4.32) holds. Then the function

$$
\rho \rightarrow \frac{\overline{\mathrm{I}}_{\rho}}{\rho} \text { is non-increasing in }\left[1-\rho_{0}, 1+1-\rho_{0}\right]
$$

Proof. For $\rho \geq \rho^{\prime}$, there holds

$$
\frac{\mathrm{II}_{\rho}(u)}{\rho}-\frac{\mathrm{II}_{\rho^{\prime}}(u)}{\rho^{\prime}}=\left(\frac{1}{\rho}-\frac{1}{\rho^{\prime}}\right)\left\langle P_{g}^{4,3} u, u\right\rangle .
$$

Therefore, it follows easily that also

$$
\frac{\overline{\mathrm{II}}_{\rho}}{\rho}-\frac{\overline{\mathrm{II}}_{\rho^{\prime}}}{\rho^{\prime}} \leq 0
$$

hence the lemma is proved. 
From this lemma it follows that the function $\rho \rightarrow \frac{\overline{\mathrm{II}}_{\rho}}{\rho}$ is a.e. differentiable in $\left[1-\rho_{0}, 1+\rho_{0}\right]$, and we obtain the following corollary.

Corollary 4.17. Let $\bar{\lambda}$ and $\rho_{0}$ be as in Lemma 4.16, and let $\Lambda \subset[1-$ $\left.\rho_{0}, 1+\rho_{0}\right]$ be the (dense) set of $\rho$ for which the function $\frac{\overline{\mathrm{II}}_{\rho}}{\rho}$ is differentiable. Then for $\rho \in \Lambda$ the functional $\mathrm{II}_{\rho}$ possesses a bounded Palais-smale sequence $\left(u_{l}\right)_{l}$ at level $\overline{\mathrm{II}}_{\rho}$.

Proof. The existence of Palais-smale sequence $\left(u_{l}\right)_{l}$ at level $\overline{\mathrm{II}}_{\rho}$ follows from (4.32) and the bounded is proved exactly as in [5, Lemma 3.2].

Next we state a proposition saying that bounded Palais-smale sequence of $\mathrm{II}_{\rho}$ converges weakly (up to a subsequence) to a solution of the perturbed problem. The proof is the same as the one of Proposition 5.5 in [14].

Proposition 4.18. Suppose $\left(u_{l}\right)_{l} \subset H_{\partial / \partial n}$ is a sequence for which

$$
\mathrm{II}_{\rho}\left(u_{l}\right) \rightarrow c \in \mathbb{R}, \quad \mathrm{II}_{\rho}^{\prime}\left[u_{l}\right] \rightarrow 0, \quad \int_{M} e^{4 u_{l}} d V_{g}=1 \quad\left\|u_{l}\right\|_{H^{2}(M)} \leq C .
$$

Then $\left(u_{l}\right)$ has a weak limit u(up to a subsequence) which satisfies the following equation:

$$
\left\{\begin{array}{cc}
P_{g}^{4} u+2 \rho Q_{g}=2 \rho \kappa_{\left(P 4, P^{3}\right)} e^{4 u} & \text { in } M, \\
P_{g}^{3} u+\rho T_{g}=0 & \text { on } \partial M, \\
\frac{\partial u}{\partial n_{g}}=0 & \text { on } \partial M .
\end{array}\right.
$$

Now we are ready to make the proof of Theorem 1.1 .

Proof of Theorem 1.1. By (4.17) and (4.18), there exists a sequence $\rho_{l} \rightarrow 1$ and $u_{l}$ such that the following holds:

$$
\left\{\begin{array}{cl}
P_{g}^{4} u_{l}+2 \rho_{l} Q_{g}=2 \rho \kappa_{\left(P 4, P^{3}\right)} e^{4 u_{l}} & \text { in } M, \\
P_{g}^{3} u_{l}+\rho_{l} T_{g}=0 & \text { on } \partial M \\
\frac{\partial u_{l}}{\partial n_{g}}=0 & \text { on } \partial M .
\end{array}\right.
$$

Now since $\kappa_{\left(P^{4}, P^{3}\right)}=\int_{M} Q_{g} d V_{g}+\int_{\partial M} d S_{g}$, then applying Corollary 1.5 with $Q_{l}=\rho_{l} Q_{g}, T_{l}=\rho_{l} T_{g}$ and $\bar{Q}_{l}=\rho_{l} \kappa_{\left(P^{4}, P^{3}\right)}$, we have that $u_{l}$ is bounded in $C^{1+\alpha}$ for every $\alpha \in(0,1)$. Hence up to a subsequence it converges in $C^{1}(M)$ to a solution of (1.4). Hence Theorem 1.1 is proved. 
Remark 4.1. As said in the introduction, we now discuss how to settle the general case.

First of all, to deal with the remaining cases of situation 1, we proceed as in [14]. To obtain Moser-Trudinger-type inequality and its improvement, we impose the additional condition $\|\hat{u}\| \leq C$ where $\hat{u}$ is the component of $u$ in the direct sum of the negative eigenspaces. Furthermore, another aspect has to be considered, that is not only $e^{4 u}$ can concentrate but also $\|\hat{u}\|$ can tend to infinity. And to deal with this, we have to substitute the set $\left(M_{\partial}\right)_{k}$ with an other one, $A_{k, \bar{k}}$, which is defined in terms of the integer $k$ (given in (1.6)) and the number $\bar{k}$ of negative eigenvalues of $P_{g}^{4,3}$, as is done in [14]. This also requires suitable adaptation of the min-max scheme and of the monotonicity formula in Lemma 4.16, which in general becomes

$$
\rho \rightarrow \frac{\overline{\mathrm{II}}_{\rho}}{\rho}-C \rho \text { is non-increasing in }\left[1-\rho_{0}, 1+\rho_{0}\right],
$$

for a fixed constant $C>0$.

As already mentioned in the introduction, see Remark, to treat the situation 1 , we only need to consider the case $\bar{k} \neq 0$. In this case, the same arguments as in [14] apply without any modifications.

\section{Acknowledgments}

I would like to thank Professor Andrea Malchiodi for having proposed to me the study of this problem and for many helpful and interesting suggestions. I am also grateful to Professor Andrei Agrachev, Sun-Yung Alice Chang and Paul Yang for fruitful discussions.

Part of this work was accomplished when I was visiting Centro De Giorgi in Pisa. I would like to thank all the members of the institute for their kind hospitality.

The author have been supported by M.U.R.S.T within the PRIN 2006 Variational methods and non-linear differential equations.

\section{References}

[1] D.R. Adams, A sharp inequality of J. Moser for higher order derivatives, Ann. Math. 128 (1988) 385-398.

[2] T. Aubin, Nonlinear analysis on manifold, Monge-Ampere equations, Springer-Verlag, 1982.

[3] T.P. Branson and B. Oersted, Explicit functional determinants in four dimensions, Proc. Amer. Math. Soc. 113-3 (1991), 669-682. 
[4] W. Chen and C. Li, Prescribing Gaussian curvatures on surfaces with conical singularities, J. Geom. Anal. 1-4 (1991), 359-372.

[5] W. Ding, J. Jost, J. Li and Wang G, Existence results for mean field equations, Ann. Inst. Henri Poincaré Anal. Non Linire 16-5 (1999), 653-666.

[6] S.Y.A. Chang and J. Qing, The zeta functional determinants on manifolds with boundary 1. The formula, J. Funct. Anal. 147 (1997), $327-362$.

[7] - The zeta functional determinants on manifolds with boundary II. Extremal metrics and compactness of isospectral set, J. Funct. Anal. 147 (1997), 363-399.

[8] S.Y.A. Chang, J. Qing and P.C. Yang, On the Chern-Gauss-Bonnet integral for conformal metrics on $\mathbf{R}^{4}$, Duke Math. J. 103-3 (2000), 523-544.

[9] S.Y.A. Chang and P.C. Yang, Extremal metrics of zeta functional determinants on 4-manifolds, Ann. Math. 142 (1995), 171-212.

[10] S.Y.A. Chang, M.J. Gursky and P.C. Yang, An equation of MongeAmpere type in conformal geometry, and four-manifolds of positive Ricci curvature, Ann. Math. 155-3 (2002), 709-787.

[11] - A conformally invariant sphere theorem in four dimensions, Publ. Math. Inst. Hautes etudes Sci. 989 (2003), 105-143.

[12] S.Y.A. Chang and P.C. Yang, On a fourth order curvature invariant.

[13] S.S. Chen, Conformal deformation on manifolds with boundary, Preprint

[14] Z. Djadli and A. Malchiodi, Existence of conformal metrics with constant Q-curvature, Ann. Math. to appear.

[15] O. Druet and F. Robert, Bubbling phenomena for fourth-order fourdimensional PDEs with exponential growth, Proc. Amer. Math. Soc. 134(3) (2006), 897-908.

[16] J.F. Escobar, The Yamabe problem on manifolds with boundary, J. Differ. Geom. 35(1) (1992), 21-84.

[17] D. Gilbar and N. Trudinger, Elliptic partial differential equations of second order, 2nd ed., Springer-Verlag, 1983. 
[18] C.B. Ndiaye, Constant Q-curvature metrics in arbitrary dimension, J. Funct. Anal. 251/1 (2007), 1-58.

[19] S. Paneitz, A quartic conformally covariant differential operator for arbitrary pseudo-Riemannian manifolds, Preprint, 1983.

[20] M. Struwe, The existence of surfaces of constant mean curvature with free boundaries, Acta Math. 160-1/2 (1988), 19-64.

[21] K. Uhlenbeck and J. Viaclovsky, Regularity of weak solutions to critical variational equations, Math. Res. Lett. 7-5/6 (2000), 651-656.

[22] X. Xu, Uniqueness and non-existence theorems for conformally invariant equations, J. Funct. Anal. 222 (2005), 1-28.

\section{SISSA}

VIA BeIRUt 2-4

34014 TRIESTE

ITALY

E-mail address: Cheikh.Birahim.Ndiaye@aei.mpg.de

RECEIVED April 13, 2007 